\title{
Standard Operating Procedures for Wild Horse and Burro Double-Observer Aerial Surveys
}

Chapter 16 of

Section A, Biological Science

Book 2, Collection of Environmental Data

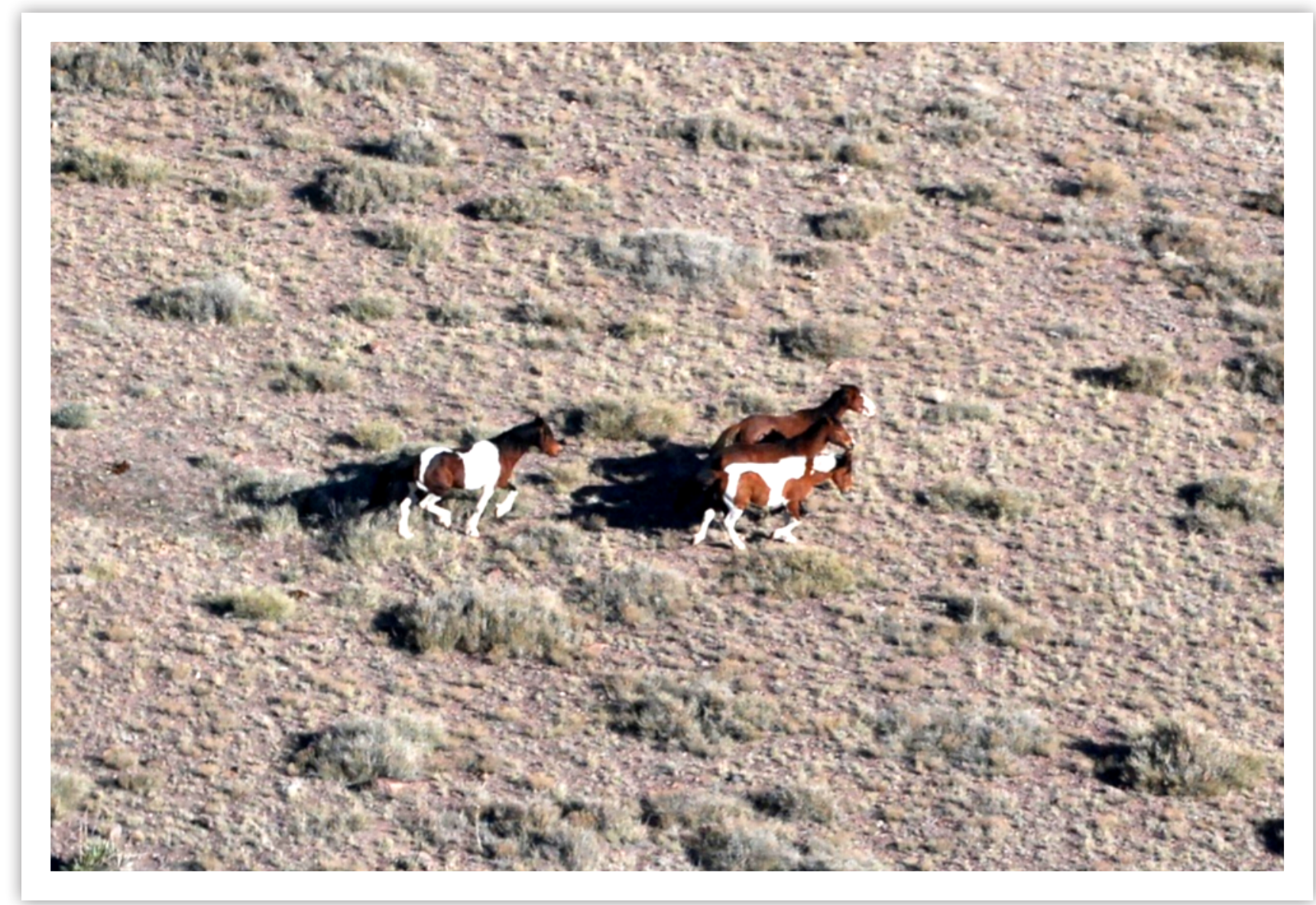

Techniques and Methods 2-A16 
Cover. Horse group photographed in Frisco Herd Management Area, Utah. Photograph by Chad Hunter, Bureau of Land Management, 2012. 


\section{Standard Operating Procedures for Wild Horse and Burro Double-Observer Aerial Surveys}

By Paul C. Griffin, L. Stefan Ekernas, Kathryn A. Schoenecker, and

Bruce C. Lubow

Chapter 16 of

Section A, Biological Science

Book 2, Collection of Environmental Data

Prepared in cooperation with the Bureau of Land Management

Techniques and Methods 2-A16 


\title{
U.S. Department of the Interior DAVID BERNHARDT, Secretary
}

\section{U.S. Geological Survey James F. Reilly II, Director}

\author{
U.S. Geological Survey, Reston, Virginia: 2020
}

For more information on the USGS - the Federal source for science about the Earth, its natural and living resources, natural hazards, and the environment-visit https://www.usgs.gov or call 1-888-ASK-USGS.

For an overview of USGS information products, including maps, imagery, and publications, visit https://store.usgs.gov.

Any use of trade, firm, or product names is for descriptive purposes only and does not imply endorsement by the U.S. Government.

Although this information product, for the most part, is in the public domain, it also may contain copyrighted materials as noted in the text. Permission to reproduce copyrighted items must be secured from the copyright owner.

Suggested citation:

Griffin, P.C., Ekernas, L.S., Schoenecker, K.A., and Lubow, B.C., 2020, Standard operating procedures for wild horse and burro double-observer aerial surveys: U.S. Geological Survey Techniques and Methods, book 2, chap. A16, 76 p., https://doi.org/10.3133/tm2A16.

ISSN 2328-7055 (online) 


\section{Preface}

The U.S. Geological Survey has been collaborating with the Bureau of Land Management to develop statistically reliable methods for wild horse and burro aerial survey data collection and analysis for more than a decade. In cooperation with Colorado State University, the U.S. Geological Survey tested several methods in herds with known abundance, resulting in two scientifically defensible aerial survey and population estimation techniques. These methods are now being applied by the Bureau of Land Management across the western United States, enabling better management of wild horses and burros. The purpose of these Standard Operating Procedures is to provide detailed instructions to the Bureau of Land Management wild horse and burro specialists who need to fly aerial surveys for management.

\section{Acknowledgments}

Many pilots, wild horse and burro specialists, survey crew members, aviation managers, and scientists contributed to the development, testing, and refinement of these methods. Jason Ransom (National Park Service) and Francis Singer, previously of the U.S. Geological Survey (USGS) Fort Collins Science Center, now deceased, were drivers behind early tests of these and other potential methods as viable approaches to wild horse and burro abundance estimation on Bureau of Land Management (BLM) lands. BLM staff from the Grand Junction, Colorado, Cody, Wyoming, and Billings, Montana, Field Offices were instrumental in early validation tests. Invaluable improvements were made through surveys carried out jointly by USGS scientists, BLM wild horse and burro specialists from throughout the west, along with cooperating U.S. Forest Service staff in comanaged complexes. We gratefully acknowledge all that work that formed the foundation of these survey methods, as well as advice and reviews of early drafts of these Standard Operating Procedures from dozens of coworkers in USGS, BLM, and U.S. Forest Service. We appreciate review by the U.S. Department of the Interior Executive Aviation Subcommittee. We appreciate guidance throughout the research and development process from Zack Bowen (USGS); Joan Guilfoyle, Dean Bolstad, and Alan Shepherd (BLM); and Tom Frolli (U.S. Forest Service). 


\section{Contents}

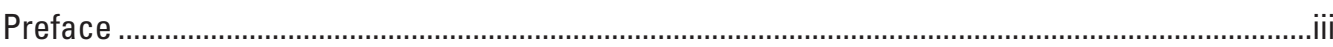

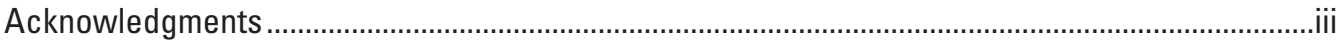

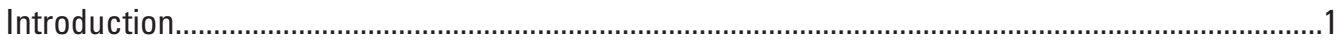

Standard Operating Procedure 1-Conducting Aerial Surveys with the Simultaneous Double-

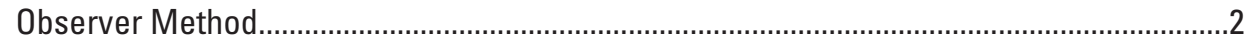

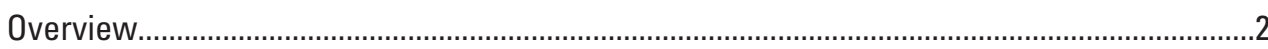

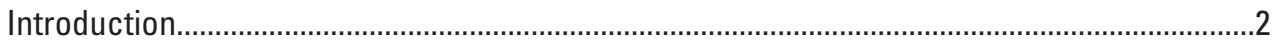

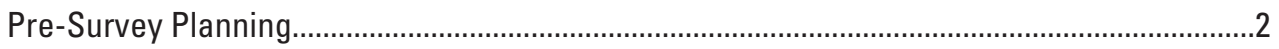

Daily Preparations and Supplies .............................................................................................

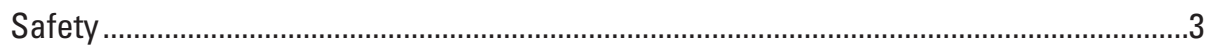

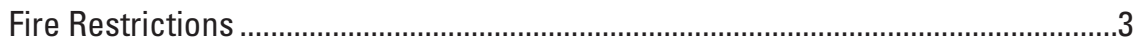

Weather and Flight .......................................................................................................

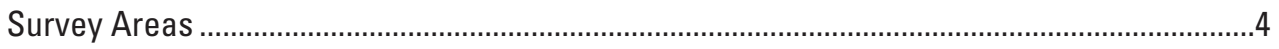

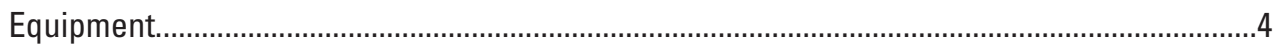

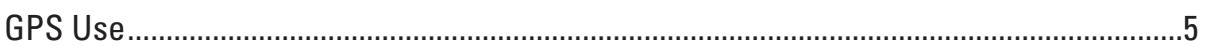

Crew Positions and Duties ...............................................................................................

Survey Procedures—How to Conduct Survey Flights ...........................................................

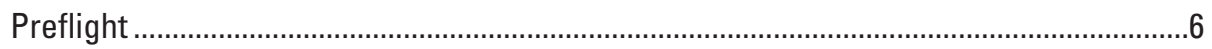

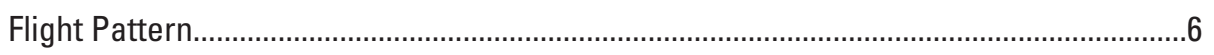

How to Search—Maintaining Observer Independence for Initial Detection....................7

Counting and Photographing Horses after Detection is Announced.............................10

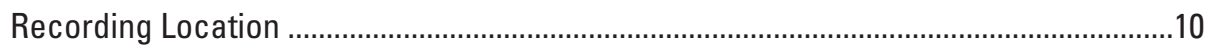

Defining Sighting Covariate Categories ....................................................................11

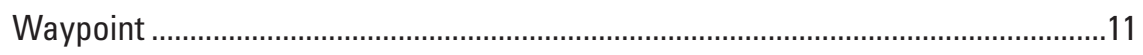

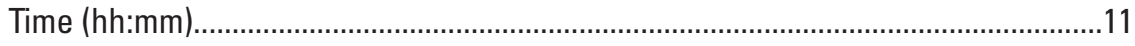

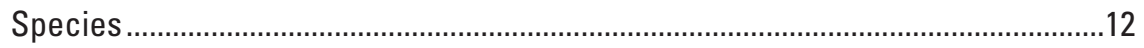

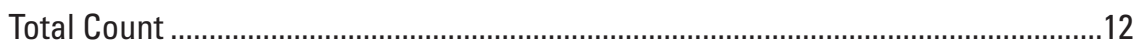

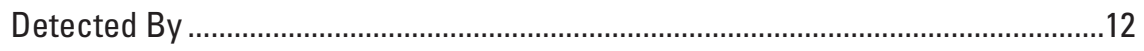

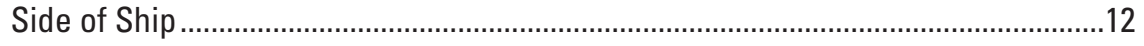

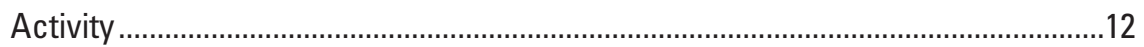

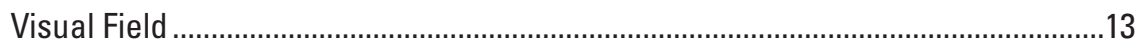

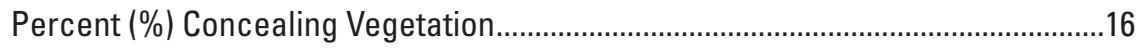

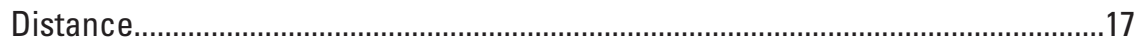

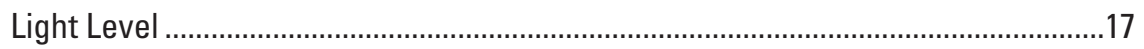

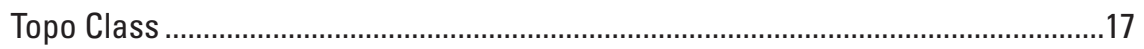

Percent (\%) of Ground Snow-Covered .............................................................18

Protocol OK?

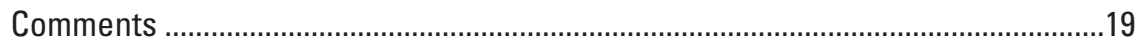

Data-Recording Shortcut—Default Values..................................................................19

What to do When the Same Group is Detected Twice..................................................19

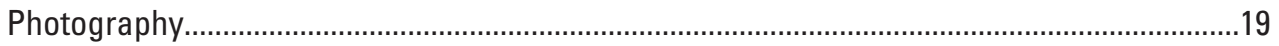

More about Recording Covariate Data, and Who Saw the Horse Group ...............................20

Special Circumstances that Will Undoubtedly be Faced..............................................20

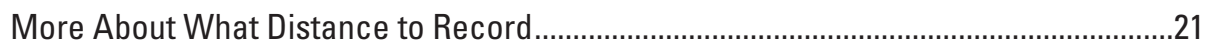




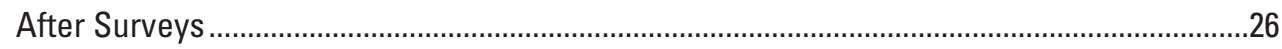

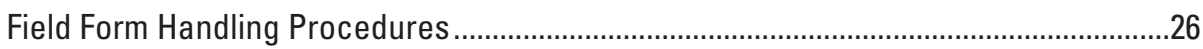

Renaming Scanned Field Data Forms ..........................................................................27

Renaming Photo and Global Positioning System Files ...................................................2

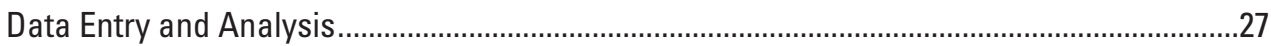

Bureau of Land Management Wild Horse and Burro Program System .........................27

Entering Double-Observer Data onto Spreadsheet ............................................................27

Sending Double-Observer Data to U.S. Geological Survey for Analysis...........................27

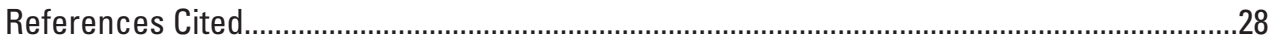

Standard Operating Procedure 2-Global Positioning System Use ...............................................29

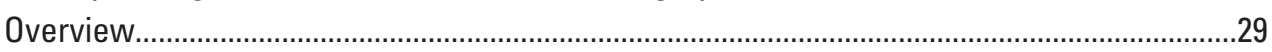

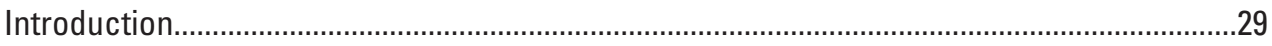

Directions for Global Positioning System Use .................................................................30

Standard Operating Procedure 3-File Folder Structure ……....................................................31

Standard Operating Procedure 4-Processing Digital Photographs..............................................32

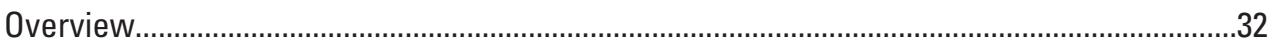

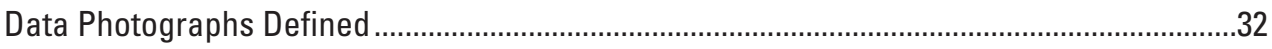

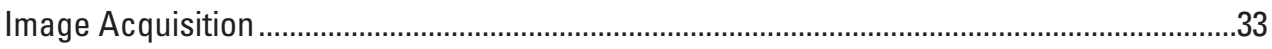

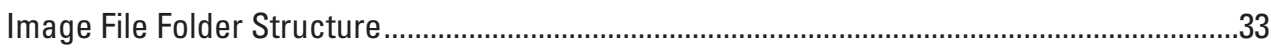

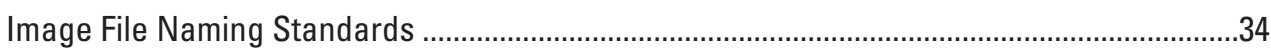

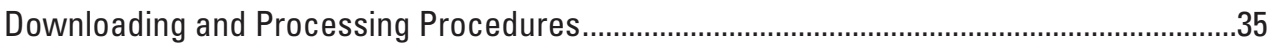

Post-Survey Cleanup Procedures and Quality Control ..........................................................38

Standard Operating Procedure 5-Reference for Analyzing Double-Observer Data .....................39

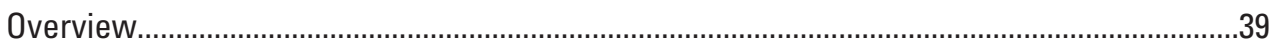

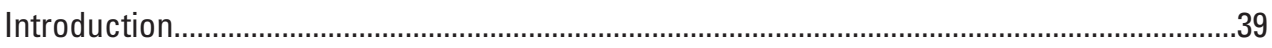

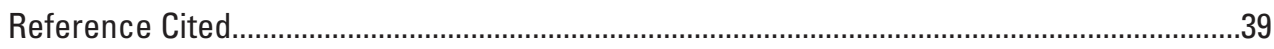

Standard Operating Procedure 6-Preparing Flight Lines for Aerial Surveys ................................40

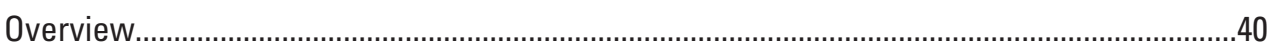

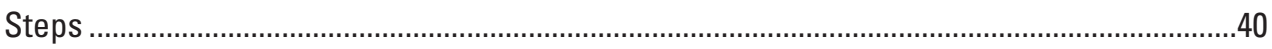

Spacing

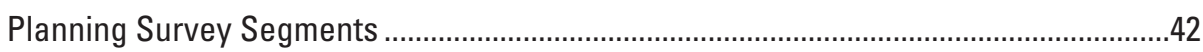

Map and Print Planned Survey Segments..................................................................42

Standard Operating Procedure 7-Principles for Projecting Population Size …............................44

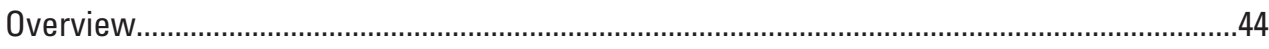

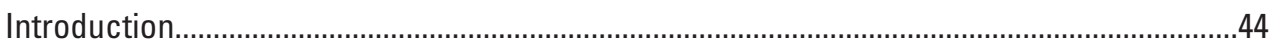

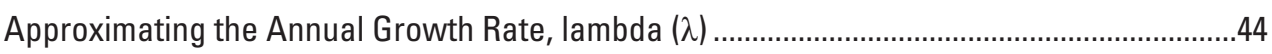

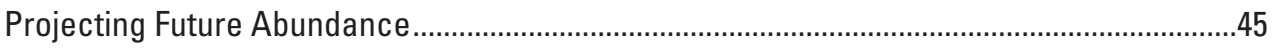

Simplest Population Projections ...............................................................................47

Projections from Population Surveys that Account for Animals Missed .........................47

Projections from Direct Counts .........................................................................................48

Projections from Post-gather Expert Opinion of the Number of Animals ........................48

Caveats About the Methods Above .......................................................................................4

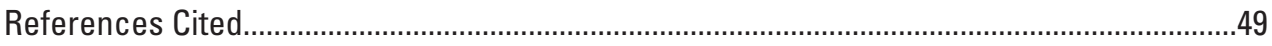

Appendix 1. List of Herd Codes, by State ...........................................................................51

Appendix 2. Examples of Percent Concealing Vegetation, $0-80$ percent ......................................61

Appendix 3. Blank Data Forms ...............................................................................................62 


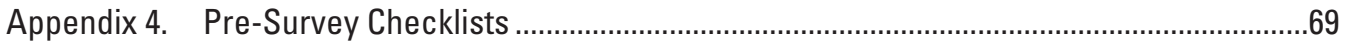

Appendix 5. Quick-Guide to Double-Observer Surveys ...................................................................

\section{Figures}

1. Hourly weather forecast graph from https://www.weather.gov ......................................5

2. A diagram of a helicopter's initial approach near a horse group ......................................8

3. A diagram of a helicopter's continued flight past a horse group .....................................

4. A diagram of the four choices for "Side of Ship" for aerial surveys ...............................13

5. A photograph of horses in a "Broken" visual field type.....................................................14

6. A photograph of a "Tree" visual field type ...................................................................15

7. A diagram of four examples of percent concealing vegetation, illustrated for a

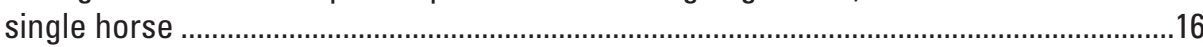

8. A diagram of two examples of percent concealing vegetation, each with a detection group of five horses...

9. An image with two examples of percent snow cover ....................................................18

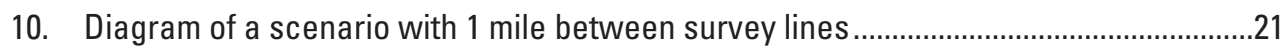

11. Diagram of an example with 1-mile transect spacing ......................................................22

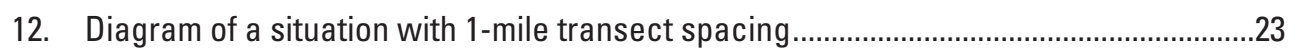

13. Diagram of a situation where extra groups are seen....................................................2

14. Diagram of a case where a horse group runs away more than 1 mile from transect....25

15. Screen snap of file folder structure for aerial survey documents for wild horse and burro surveys.

16. Screen snap of an example file folder structure for wild horse and burro aerial

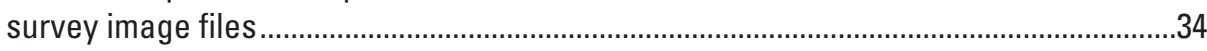

17. Screen snap of an example file folder structure for wild horse and burro aerial survey image files for downloading and processing procedures .....................................35

18. Screen snap of an example file folder structure for wild horse and burro aerial survey image files for downloading and processing procedures ....................................36

19. Screen snap of an example file folder structure for wild horse and burro aerial survey image files for downloading and processing procedures ...................................36

20. Image with diagram of flight lines for aerial surveys for wild burros ...............................43

21. Flow chart for projecting population size for wild horses and burros..............................46

\section{Table}

1. Preparing flight lines for wild horse and burro double-observer aerial surveys 


\section{Conversion Factors}

U.S. customary units to International System of Units

\begin{tabular}{|c|c|c|}
\hline Multiply & By & To obtain \\
\hline \multicolumn{3}{|c|}{ Length } \\
\hline foot $(\mathrm{ft})$ & 0.3048 & meter $(\mathrm{m})$ \\
\hline yard (yd)) & 0.9144 & meter $(\mathrm{m})$ \\
\hline mile (mi) & 1.609 & kilometer (m) \\
\hline mile, nautical (nmi) & 1.852 & kilometer $(\mathrm{km})$ \\
\hline \multicolumn{3}{|c|}{ Velocity } \\
\hline knot (kn) & 1.852 & kilometers per hour $(\mathrm{km} / \mathrm{hr})$ \\
\hline
\end{tabular}

International System of Units to U.S. customary units

\begin{tabular}{lll}
\hline \multicolumn{1}{c}{ Multiply } & By & \multicolumn{1}{c}{ To obtain } \\
\hline meter $(\mathrm{m})$ & Length & foot $(\mathrm{ft})$ \\
meter $(\mathrm{m})$ & 3.281 & yard $(\mathrm{yd})$ \\
kilometer $(\mathrm{km})$ & 1.094 & mile $(\mathrm{mi})$ \\
kilometer $(\mathrm{km})$ & 0.6214 & mile, nautical $(\mathrm{nmi})$ \\
\hline & 0.5400 & \\
\hline kilometers per hour $(\mathrm{km} / \mathrm{hr})$ & Velocity & knots $(\mathrm{kn})$ \\
\hline
\end{tabular}

1 nautical mile $(\mathrm{nmi})=1.1508$ statute miles $(\mathrm{mi})$

1 knot $(\mathrm{kn})=1.15$ statute miles per hour $(\mathrm{mi} / \mathrm{hr})$

1 knot $(\mathrm{kn})=1.15078$ nautical miles per hour (nmi/hr)

Temperature in degrees Celsius $\left({ }^{\circ} \mathrm{C}\right)$ may be converted to degrees Fahrenheit $\left({ }^{\circ} \mathrm{F}\right)$ as

$$
{ }^{\circ} \mathrm{F}=\left(1.8 \times{ }^{\circ} \mathrm{C}\right)+32 \text {. }
$$

Temperature in degrees Fahrenheit $\left({ }^{\circ} \mathrm{F}\right)$ may be converted to degrees Celsius $\left({ }^{\circ} \mathrm{C}\right)$ as ${ }^{\circ} \mathrm{C}=\left({ }^{\circ} \mathrm{F}-32\right) / 1.8$.

\section{Datum}

Horizontal coordinate information is referenced to the North American Datum of 1983 (NAD 83).

Altitude, as used in this report, refers to the height above ground level; this is the distance above the vertical datum. 


\section{Abbreviations}

$\begin{array}{ll}\text { AFF } & \text { automated flight following } \\ \text { AGL } & \text { elevation above ground level } \\ \text { BLM } & \text { Bureau of Land Management } \\ \text { FAA } & \text { Federal Aviation Administration } \\ \text { GIS } & \text { geographic information system } \\ \text { GPS } & \text { Global Positioning System } \\ \text { HA } & \text { herd area } \\ \text { HMA } & \text { Herd Management Area } \\ \text { IHOG } & \text { interagency helicopter operating guidelines } \\ \text { NWCG } & \text { National Wildfire Coordinating Group } \\ \text { PASP } & \text { Project Aviation Safety Plan } \\ \text { PPE } & \text { personal protective equipment } \\ \text { SOP } & \text { standard operating procedure } \\ \text { USFS } & \text { U.S. Forest Service } \\ \text { USGS } & \text { U.S. Geological Survey } \\ \text { VFR } & \text { visual flight rules } \\ \text { WHB } & \text { wild horse and burro } \\ \text { WHT } & \text { Wild Horse Territory }\end{array}$

\section{Useful Websites}

https://fsapps.nwcg.gov/afm/ Active fire mapping website with the status of local forest fires and locations of temporary no-fly zones

https://skyvector.com Layers that can be turned on to show wind barbs and icing hazards overlaid on Federal Aviation Administration sectional maps

https://www.blm.gov/services/national-operations-center Bureau of Land Management National Operations Center

https://www.weather.gov The National Weather Service forecast office providing rainfall probability and wind speed predictions for any given location 


\title{
Standard Operating Procedures for Wild Horse and Burro Double-Observer Aerial Surveys
}

\author{
By Paul C. Griffin, ${ }^{1}$ L. Stefan Ekernas, ${ }^{2}$ Kathryn A. Schoenecker, ${ }^{3}$ and Bruce C. Lubow ${ }^{4}$
}

\section{Introduction}

This report provides multiple standard operating procedures (SOPs) that are related to Equus caballus (wild horse) and Equus asinus (wild burro) double-observer aerial surveys, along with datasheets, pre-survey checklists, and a quick-guide to the methods.

SOP 1 describes how to carry out wild horse and burro (WHB) aerial surveys as an aviation crew member. SOP 2, SOP 3, and SOP 4 relate to data management, and are important for the WHB specialist or other lead staff who will be responsible for documenting and archiving records from the survey. SOP 5 details double-observer reporting via a data entry spreadsheet and provides reference for analyzing double observer data to obtain population estimates. SOP 6 presents general principles for preparing aerial survey flight lines. SOP 7 provides instructions for using abundance estimates from aerial surveys to project population size forward in time. The appendixes provide survey datasheets, pre-survey checklists, and a quick-guide to SOP 1.

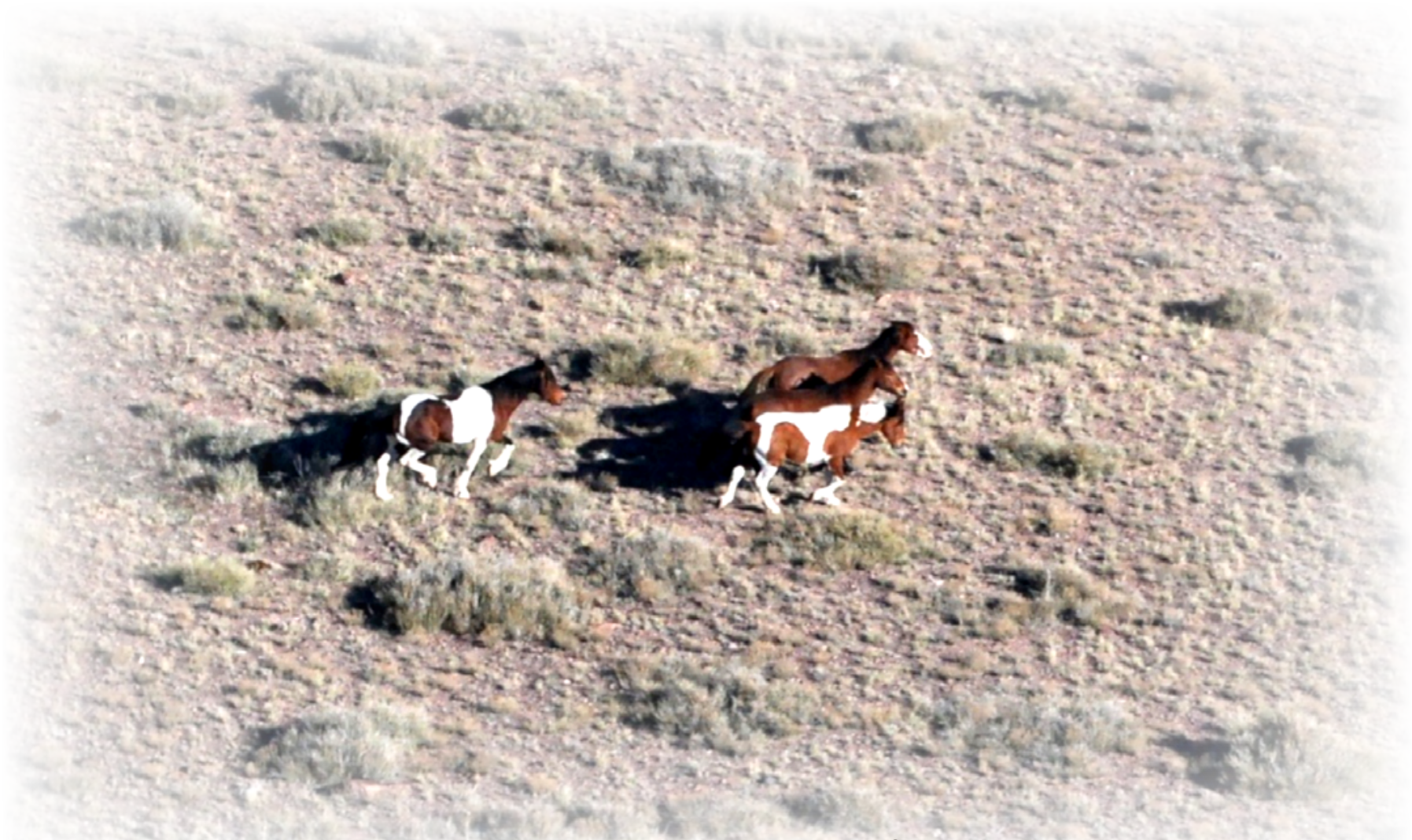

Horse group photographed in Frisco Herd Management Area, Utah. Photograph by Chad Hunter, Bureau of Land Management, 2012.

${ }^{1}$ Bureau of Land Management.

${ }^{2}$ Denver Zoo (formerly with the U.S. Geological Survey).

${ }^{3}$ U.S. Geological Survey.

${ }^{4}$ Colorado State University. 


\section{Standard Operating Procedure 1-Conducting Aerial Surveys with the Simultaneous Double-Observer Method}

\section{Overview}

This SOP provides instructions for conducting aerial surveys with the "simultaneous double-observer method." This method can also be called the "double-observer" method; the name refers to the fact that two or more observers are trying to detect groups independently. After everyone has an independent chance to detect a given "detection group," the observers work together to describe it. Each detected group only gets recorded once.

Sometimes people refer to this method as the "double-count" method. In this SOP we only use the phrase "double-count" to mean a group of horses that is recorded twice in a data set. Crew members should take measures (listed in these SOPs) to correctly identify any groups that have already been recorded, so that such groups only are counted once in the total number of animals present.

In terms of the analysis, the double-observer method is a form of mark-resight abundance estimation where the front seat observer is considered one independent survey, and the rear seat observer acts as a second independent survey. Throughout this SOP, the word "survey" also means "inventory." This SOP refers to horses, but the same methods may be used to collect data on burros. There are only slight differences in how data are recorded for burros, having to do with the measure of burro distance from the transect line.

\section{Introduction}

Surveys are carried out to maximize the chance of seeing the largest number of horses present, in a short period of time, and to record the data in a way that allows for statistical estimation of the number of horses and burros that were present but not seen. Each observer acts independently of other observers in the initial detection of horse groups. No communication, visual or verbal, can occur between front and rear observers until after both observers have had an adequate independent opportunity to detect each horse group. If the pilot spots a horse group, he/she should also wait to identify that group to the front observer. Unless there is a good chance the groups will escape before being counted (see below, in the section "Special Circumstances that Will Undoubtedly be Faced"), it is essential that the rear observers are not alerted until after the helicopter has passed the group.

The pilot and front observer need to wait until the horse group has passed abeam of the aircraft (that is, past the rear observer's shoulder) to be sure the rear observers have an adequate chance to see the group. After a group is abeam of the aircraft, observers can notify the entire crew if they feel it is necessary to circle the group to obtain a better count or classify the group as to adults and foals. After the group has been announced, all crew members can talk and collaborate in the count and description of the group. One crew member takes digital photographs of large groups ( 20 or more) for more careful recounting after the flight. By moving the helicopter between the horses and transects that have not yet been surveyed, at a safe elevation above ground level (AGL), the pilot can gently move horses away from those future transect lines to avoid re-counting groups. However, any such "double-counted" groups will only be included once in the total estimate of herd size.

\section{Pre-Survey Planning}

In all cases, it is essential to have a flight plan that is safe and clearly understood by all participants. District WHB specialists coordinate with state leads to identify the Herd Management Areas (HMAs) and complexes that are slated for survey. HMAs and the surrounding areas that horses use are typically the areas being surveyed. This SOP mainly refers to HMAs, but it should be understood that U.S. Forest Service (USFS) Wild Horse Territories (WHTs), Bureau of Land Management (BLM) herd areas (HAs), or other lands may be the area of interest for some surveys. For complexes involving more than one district, or which span both BLM and USFS jurisdiction, additional coordination across agencies and (or) states will be needed. Follow these guidelines when planning the route and timing of surveys:

- Plan for complete and systematic coverage of the entire HMA, HA, or complex, plus any areas outside those boundaries that horses from that herd potentially utilize.

- Lay out transects ahead of time and load them into the pilot's Global Positioning System (GPS) unit for the aircraft to follow. This maximizes the search effort for crew members and reduces risk. Transects should be planned to minimize exposure to hazards such as transmission lines and towers. 
- Complete survey activities at, or close to, the same time of year for each individual HMA or complex, to reduce seasonal variability in population estimates. Late summer or early fall surveys can generally be used to obtain estimates of foal-toadult ratios.

- Inventory HMAs, HAs, and WHTs within complexes at the same time using the same methods and observers rather than inventorying HMAs within a complex separately or at different times, using different methods and observers.

- Combine surveys of several small HMAs, even if they are somewhat distant from each other, using the same crew in order to achieve appropriate sample size. A good minimum sample size for effective statistical analyses is at least 60 horse group observations. Sometimes observations can be combined from two or more years if observers and conditions are mostly consistent.

- InterDistrict, inter-State, and inter-Agency coordination is mandatory in order to have accurate population estimates on those HMAs or Complexes that cross District, State, and Federal land ownership boundaries.

- Use natural or manmade barriers as opposed to administrative boundaries to define inventory units.

- Schedule three aircrew members/observers. Preferably, have a back-up aircrew member/observer.

Simultaneous double-observer surveys can be conducted with either a helicopter or a fixed wing aircraft, as long as there is room for the pilot plus three aircrew members/observers, and adequate visibility from the windows for all (Ransom, 2012). Helicopters can fly slowly and low (below 500 feet [ft] AGL), so they are more suitable for mountainous topography and (or) areas with extensive amounts of vegetation that could conceal horses. Airplanes generally fly faster, and must stay greater than or equal to $(\geq) 500$ feet AGL, so they are more suitable in areas with flat to rolling topography with little concealing vegetation. For either type of aircraft, the portion of flights to and from surveyed areas should be at safe visual flight rules (VFR) cruising altitudes that would allow the pilot adequate time to react to mechanical problems.

Simultaneous double-observer surveys require three observers and a pilot. U.S. Department of the Interior and USFS surveys also require a helicopter manager or fixed-wing flight manager. Observers should read and be familiar with this SOP (especially the "Safety" and "Survey Procedures-How to Carry Out Survey Flights" sections, below). Preferably, no more than one inexperienced aircrew member would be on any single survey flight.

\section{Daily Preparations and Supplies}

\section{Safety}

Safety is essential for all aspects of every survey. The brief notes below are no substitute for careful and complete compliance with all departmental and Agency aviation safety guidelines and policies. Any flight with a Federal employee on board must follow all applicable aviation policy, including interagency helicopter operating guidelines "IHOG" (National Wildfire Coordinating Group [NWCG], 2019). For BLM/USFS flights, the WHB specialist should prepare and secure approval for the Project Aviation Safety Plan (PASP) well in advance (see Action Checklist for Horse Surveys in app. 4). The PASP should be understood by all flight crew members and helicopter manager. One copy of the PASP, including maps of intended survey routes, should be distributed ahead of time to (1) dispatchers who will be flight following, (2) aircraft manager, and (3) one onboard the aircraft.

Cooperative Tribal or State flights with no Federal staff on board are not necessarily subject to U.S. Department of the Interior aviation regulations. However, any flights happening in the same time and in the vicinity of each other should be coordinated.

On any survey when two aircraft are scheduled to conduct surveys at the same time, for safety reasons, no aircraft shall survey unless both aircraft are in communication with each other and with the shared dispatch.

\section{Fire Restrictions}

Information on flight restrictions due to forest fires should be obtained daily. Crew leaders and pilots can check the status of local forest fires and locations of temporary no-fly zones at the following USFS active fire mapping website: https://fsapps.nwcg.gov/afm/.

Depending on the region, there may be military airspace restrictions or additional safety requirements that must be addressed well ahead of time, according to State or National Agency guidelines; consult the local or state aviation manager. The PASP is required to address any restricted airspace and ensure that airspace de-confliction has been addressed by dispatch prior to flight. 


\section{Weather and Flight}

Many factors influence the go/no-go decisions on whether to proceed with any given survey. The pilot or any aircrew member can direct surveys to stop if conditions are not safe.

Helicopters hired by BLM or USFS, or with any BLM or USFS employees on board may not survey if wind speed exceeds 30 knots (55 kilometers per hour [km/hr] or 34 miles per hour [miles/hr]) of steady wind or if the wind gust spread exceeds $15 \mathrm{knots}(27 \mathrm{~km} / \mathrm{hr}$ or $17 \mathrm{miles} / \mathrm{hr}$ ), as per interagency helicopter operating guidelines (NWCG, 2019) guidelines.

Do not survey when rain or snow is falling steadily. A light rain or drizzle may be acceptable for surveys, but rain, snow, and wet windows could affect flight safety by impairing the crew's ability to see flight hazards and to conduct a quality survey.

There should be little to no ground fog or clinging mountain mists for surveys. Fog and mist can be a safety hazard that also obscures the survey area. Fog is especially opaque in bright light conditions. Ground fog may be heavy on a cool morning after a heavy rain.

The National Weather Service Forecast office provides rainfall probability and wind speed predictions for any given location.

1. Go to https://www.weather.gov; in the field for "Local forecast by "City, St" type a location near the survey area, such as "Rangely, CO."

2. Click on the "Get Detailed Info" link. Choose a location for the Detailed Point Forecast in the survey area, using the Google map window at right.

3. Once the forecast is shown for that location, scroll to the bottom of the page and select "Hourly Weather Graph" from the Additional Forecasts \& Information menu. The hourly weather forecast graph includes predicted temperatures, wind speeds, and precipitation probability (fig. 1).

Another website with a good interface for planning aviation operations is https://skyvector.com; this site has layers that can be turned on to show wind barbs and icing hazards overlaid on Federal Aviation Administration (FAA) sectional maps.

Be aware of impending storms; consider releasing the helicopter early enough that the helicopter can return to its base before any long storm arrives. This can avoid the expenses that come if the pilot and aircraft are forced to stay grounded for several days; in addition to per diem, costs may include three or more hours of flight time per day, depending on the contract.

\section{Survey Areas}

Plan survey flights ahead of time with pre-defined flight paths. Flight plans ensure that the designated survey area will be completely covered and free up observers to focus only on finding horses and burros.

Having the survey areas separated into units that are bounded by ridges, fences, and (or) major highways helps to limit the potential for horses to move between survey areas between flights. Use these breaks between units as a convenient time to refuel.

If there are ever two aircraft surveying in the same complex, survey crews must coordinate flight plans in advance so there is no question about which aircraft is surveying in which exact areas. This is critically important if two helicopters are surveying in a complex at the same time. The geographic information system (GIS) Specialist for the district or state should maintain digital files of the areas that are to be surveyed as polygon features or in a geodatabase.

The pilot should be able to identify the planned and actual flight paths, in real time, during a survey. Having paper maps in addition to the GIS/GPS enabled laptop allows for navigation if all electronics should fail. The GIS Specialist should print several sets of planned flight line maps; one set should be given to dispatch prior to the survey, one set should be brought on the aircraft, and one set should be left with the aircraft manager.

\section{Equipment}

The WHB specialist or the USFS specialist should confirm that all survey equipment is ready. In addition to a helicopter and pilot, the main items needed include personal protective equipment (PPE), datasheets, clocks or watches, a GPS unit or GPSenabled device for recording data (that is, Trimble Yuma, GPS-enabled laptop, or other), at least one additional GPS unit to serve as a backup for recording flight lines, and a digital camera. Detailed lists of required equipment are presented in the Checklist for Survey Equipment (app. 4)

Pilots should be accustomed to using GPS units for navigating along planned transect lines. The district or state GIS specialist, WHB specialist, U.S. Geological Survey (USGS) aerial survey specialist, or other staff should be prepared to upload the planned flight lines to the pilot's GPS unit before flights. General instructions for uploading to the pilot's GPS unit are in SOP 2. 


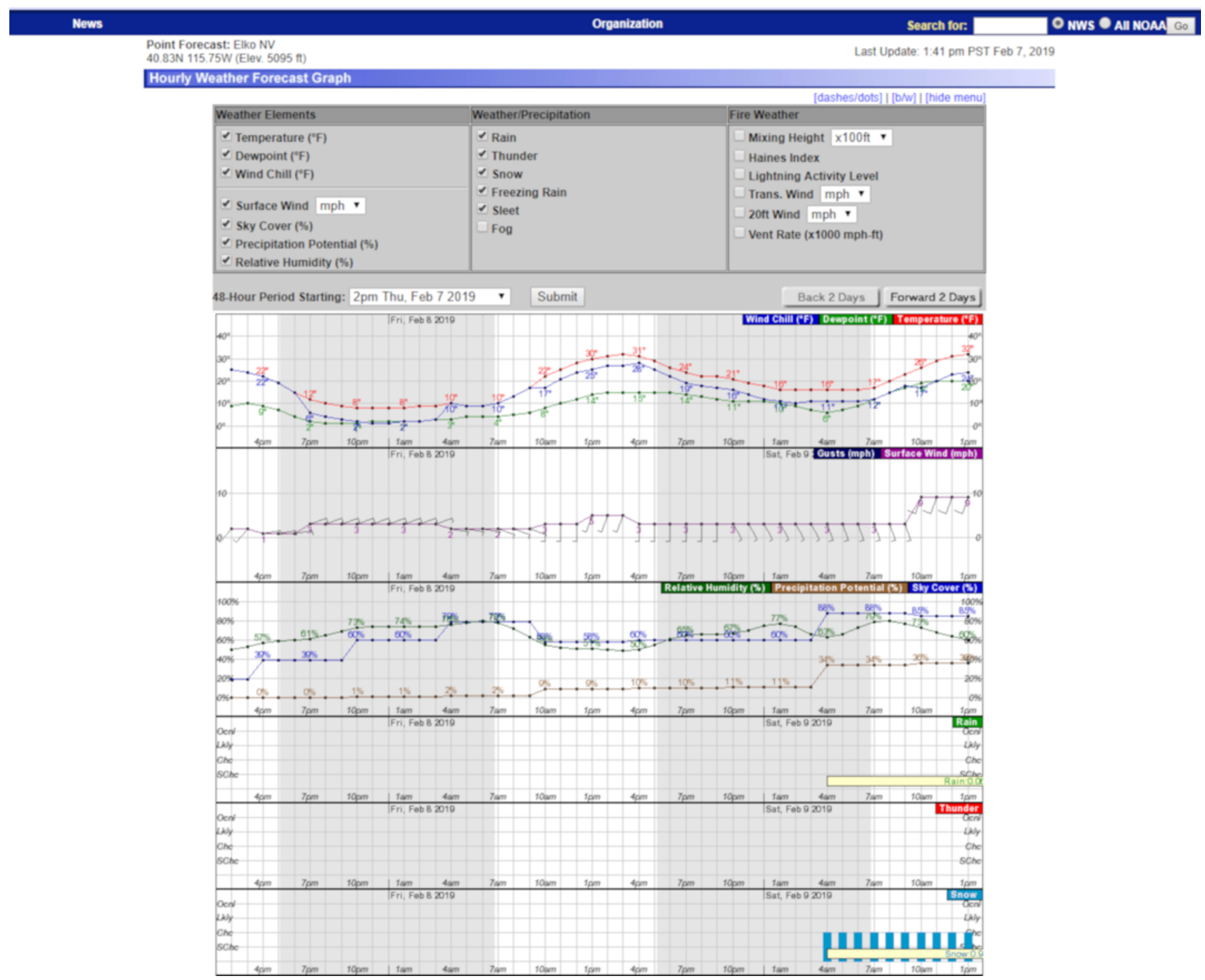

Figure 1. Hourly weather forecast graph from https://www.weather.gov. Average wind speed is shown as a purple line in the graph. Wind barb symbols represent the wind speed and direction; each full barb represents an additional 10 knots (18.5 kilometers per hour $[\mathrm{km} / \mathrm{hr}])$, and each half barb represents $5 \mathrm{knots}(9 \mathrm{~km} / \mathrm{hr})$. Rainfall/snowfall probability are shown with bars indicating small chance, chance, likely, or occasional. Total expected rainfall in different time periods is printed across the bottom of the rain graph.

\section{GPS Use}

At this time, uploading requires a laptop with the planned flight lines saved as a tracklog or shapefile that is compatible with the pilot's GPS unit. In some cases, proprietary software is needed that is compatible with the GPS unit. For example, if the unit is a Garmin, a working copy of Garmin's software, ExpertGPS, DNRGarmin (Minnesota Department of Natural Resources, 2008), or similar software is needed, along with and a universal serial bus (USB) to mini-USB cable.

On each survey flight, the pilot's GPS (or a laptop or other device with mapping software) should show and record the planned and actual flight lines. Having this device is essential for knowing where the helicopter is, for making sure that survey coverage is adequate, and for logging the flight lines in detail (that is, one point per 1-2 seconds). The crew must record a waypoint of each observed group of horses; this documents the survey results and helps to determine whether a group has been recorded previously. It is typical to use the pilot's GPS device for mapping and a second GPS unit for marking waypoints at horse group locations. In some systems the pilot can mark horse group locations as waypoints, but this should only be done if marking waypoints does not require distraction from the pilot's primary duty - flying the aircraft safely. If a laptop or tablet-type device is used, the choice of GPS model and software is left to the district, but it should be connected to a GPS unit, or be otherwise GPS-enabled. 
At least one additional GPS unit (separate from the one used to mark waypoints) should record the entire flight as a backup, in case the pilot's GPS fails to record the flight path. The flight line should be recorded at $1-2$ second intervals. The pilot's GPS unit can serve this purpose if it is configured to do so before flight, and it has enough memory to record the flight track file.

The most common setup for GPS devices will be (1) the pilot's GPS device used for navigation and to record one copy of the actual flight path flown; (2) a handheld GPS used by a crew member to mark waypoints and record the main copy of the flight path flown; and (3) a second handheld GPS, secured in an unobtrusive location, to record a backup version of the flight path flown.

\section{Crew Positions and Duties}

The crew consists of one pilot, one front seat aircrew member/observer, and two back seat aircrew member/observers. The three observers each have one assignment in addition to searching for horse groups. One observer records written data on the forms, and this person should do this consistently between days since recording data decreases detection probability. The second observer records GPS waypoints and calls out waypoint numbers to the data recorder. The third observer takes photographs and videos.

The pilot's primary duty is to fly the helicopter safely and to navigate. The pilot may also inform the aircrew members/ observers about any horse group observations he or she makes, but the pilot effort should be consistent throughout the flight. In contributing observations to the survey, the pilot should follow the same double-observer methods that relate to the timing of calling out groups as other crew members - namely, the pilot should not alert aircrew members/observers about horse groups until the helicopter has passed abeam of the group.

The position of the two back seat aircrew members/observers should switch at every refueling break, to allow us to estimate each backseat observer's detection probability compared to the front seat observer.

\section{Survey Procedures—How to Conduct Survey Flights}

\section{Preflight}

The helicopter and crew should arrive at the airport or helispot at least one hour before takeoff to allow for check-in by the aircraft manager, prepare the flight manifest and load calculations, radio frequency checks, and conduct safety and mission briefings. Along with all other required and mission-specific safety briefing items, the crew shall review the following: the pilot's central mission being to focus on conducting safe flight; the importance of situational awareness and crew resource management for all involved; the VFR cruising flight altitude above ground level that will be maintained to and from survey areas; and confirmation that all aircraft gauges are operating properly. Pre-flight is also the time for fueling, any electronic equipment installation, a group review of covariate categories, last-minute synchronizing watches to GPS units, and window cleaning. A crew member may need to upload the planned flight lines onto the pilot's GPS unit (SOP 2. GPS Use) if this has not been done already.

Before takeoff, the data recorder fills in the Flight Information Form (see app. 3) including date, area surveyed, and descriptions of aircraft and crew. There should be one new Flight Information Form completed for each day of flights. Before each takeoff, the data recorder also fills in a section of the Flight Information Form with a new flight number, seating chart, descriptions of weather (see the section in app. 3, "Completing the Flight Information Form") and the takeoff time. The pilot calls dispatch immediately after takeoff to relay the aircraft's destination, heading to next check point, the number of people onboard, the estimated hours of fuel, and to confirm that automated flight following (AFF) is operational. The pilot calls dispatch at the time of arrival to the designated survey area. When the aircraft arrives at the area to be surveyed, the data recorder enters the Survey Start time on the Flight Information Form.

\section{Flight Pattern}

The pilot flies the aircraft following pre-planned flight lines designed so that the area of interest is completely surveyed as per BLM policy (BLM, 2010). For helicopter surveys the preferred AGL is between about ( ) 150' to 400', and for fixed wing surveys the preferred AGL is just above 500'. SOP 6 (Preparing Flight Lines for Aerial Surveys) provides example flight line planning, for example with parallel flight lines over thicker vegetative cover (dense shrubs and trees) spaced at $\sim 300$ meters (m) $-600 \mathrm{~m}(0.16-0.32$ nautical mile [nmi]). This distance may be wider, depending on the openness of the habitat. Rarely would flight lines be spaced wider than 1.5 miles (mi), unless the habitat is extremely open, free of vegetation, and all observers are known to have excellent acuity. Groundspeed and flight pattern will vary depending on topography and forest canopy density. Ground speed will vary depending on the species being counted, the aircraft model and topography, but may average 50-60 knots for burro helicopter surveys, 55-70 knots for horse helicopter surveys, and 95-120 knots for fixed wing horse surveys. The front seat aircrew member/observer, or the aircrew member/observer with the GPS unit, should periodically check groundspeed and suggest changes if needed. 
Surveyed areas must be consistent over many years. The entire HMA (or complex) must be surveyed, unless there is a statistically justifiable stratified sampling design used. Horse (or burro) groups that are found outside of the planned survey area should also be recorded but they should be noted in the Comments box. If it is known that an observed group is outside the HMA, then note that clearly in the comments field of the appropriate WHB Survey Datasheet (app. 3). If it isn't known, that is okay; the waypoint location and time stamp for the observation will be mappable relative to the HMA boundary. Horse groups observed outside of planned survey areas will be reported separately in population estimates.

\section{How to Search—Maintaining Observer Independence for Initial Detection}

Each aircrew member/observer needs to have opportunity to detect each horse group independent of other observers. Each observer allows other observers to have an adequate opportunity to independently detect each horse group, and observers who detected the groups independently are recorded as "Who Saw" the horse group (see app. 3, "Instructions for Survey Datasheet" for either horses or burros). Collecting data in this way allows a statistician to compute detection biases based on the independent observations of individual aircrew members (Schoenecker and others, 2006; Ransom, 2012; Lubow and Ransom, 2016).

When any aircrew member/observer sees a horse group, she or he starts to observe attributes of that group, and may note them mentally, or on paper. That aircrew member/observer should not, however, alert others verbally or with motions until the helicopter has moved past a point where the horse group is perpendicular to the flight path (that is, when an imaginary line from the horse to the helicopter is past perpendicular to the flight path, that is, $\sim 100$ degrees relative to the nose of the ship). At that time, anyone who has seen the animal(s) says so and calls out "clock directions" to the pilot to obtain the best possible view of the group (that is, "Single horse at 3 o'clock."). Clock directions are based on the directions pointed by a clock's hands, oriented with 12 o'clock straight ahead of the aircraft, and 3 o'clock perpendicular to the right, and 9 o'clock perpendicular to the left (see the quick-guide in app. 5). The pilot then maneuvers the aircraft to the animal(s) and the flight crew works together to count the group and record covariates. The pilot may not need to divert from the flight path if the horse group is small and plainly visible. The data recorder fills in the time, count, and covariates (see app. 3, the "Instructions for Survey Datasheets"). The person operating the GPS unit marks a waypoint and announces the waypoint number.

To illustrate, in figures 2 and 3, the fields of view for the four aircrew members/observers are suggested by colored shading: yellow for the aircrew member/observer in the right back seat, red for the aircrew member/observer in the right front seat, blue for the aircrew member/observer in the left front seat, and purple for the aircrew member/observer in the left back seat. The blue line suggests an axis that is perpendicular to the helicopter flight path. When horses are behind this axis, they are said to be "past perpendicular," or "abeam" of the flight path.

In figure 2, the horse group is still in front of the helicopter; it is not yet abeam. At the time represented in figure 2, no one should mention the horse group, and the pilot should continue to fly straight along the planned plight path. At the time represented in figure 3, though, the majority of the horses in the group are abeam or behind the helicopter; the group is "past perpendicular." At the time represented in figure 3, anyone who has seen the horse group should say so, and should help guide the pilot to the group, so that the entire crew can then work together to count the group and record other covariates. When groups are detected so that observers have an opportunity to independently detect horse groups, this is recorded as "Protocol OK" on the appropriate Survey Datasheet.

Sometimes, the front seat aircrew member/observer (or pilot) may see a horse group as the horses are running into vegetation or terrain where they will be very difficult to count. The aircrew member/observer (or pilot) may decide to alert the rest of the crew about that horse group immediately, to increase the accuracy of the count of the horse in that group. If this happens, the data recorder should indicate that the back seat aircrew members/observers did not have an adequate opportunity to detect the horse group (by circling "no" in the Protocol OK column; see the appropriate "Instructions for Survey Datasheet"), but remember to record all the covariates for every group detected. Data from this kind of observation are essential in estimates of abundance. Groups that are not "Protocol OK" will not be used to fit models of detection probability, but the number of adults, foals, and all other covariates need to be recorded for every group so that the models can be applied.

Note that if one of the aircrew members/observers, such as the local WHB specialist or range conservation officer, knows that the aircraft is approaching an area that historically has a high concentration of horses such as a water source or spring then that person should share their knowledge ahead of time with the rest of the aircrew members/observers. In that way, the process of detection can remain strictly a visual process, and not be improved for just one person by their private knowledge of the place. 


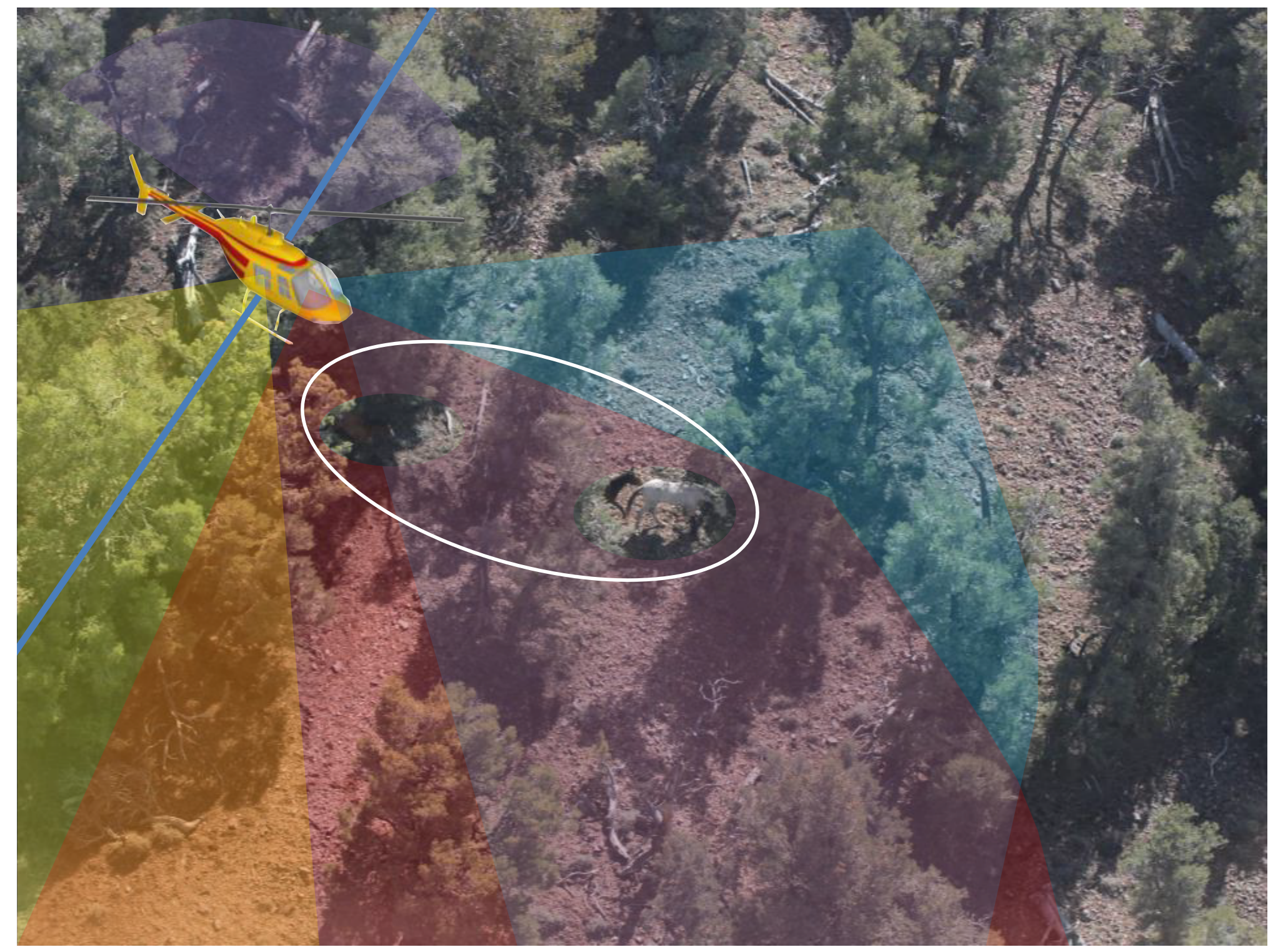

Figure 2. A diagram of a helicopter's initial approach near a horse group, circled in white. The horse group is visible to front seat observers, but back seat observers may not yet have had a chance to detect the horse group. At this point, the front seat observer and pilot should stay silent, and the pilot should keep flying straight. The fields of view for the four aircrew members/observers are suggested by colored shading: yellow for the aircrew member/observer in the right back seat, red for the aircrew member/pilot in the right front seat, blue for the aircrew member/observer in the left front seat, and purple for the aircrew member/observer in the left back seat. The blue line suggests an axis that is perpendicular to the helicopter flight path. When horses are behind this axis, they are said to be "past perpendicular," or "abeam" of the flight path. Photograph by Bureau of Land Management. 


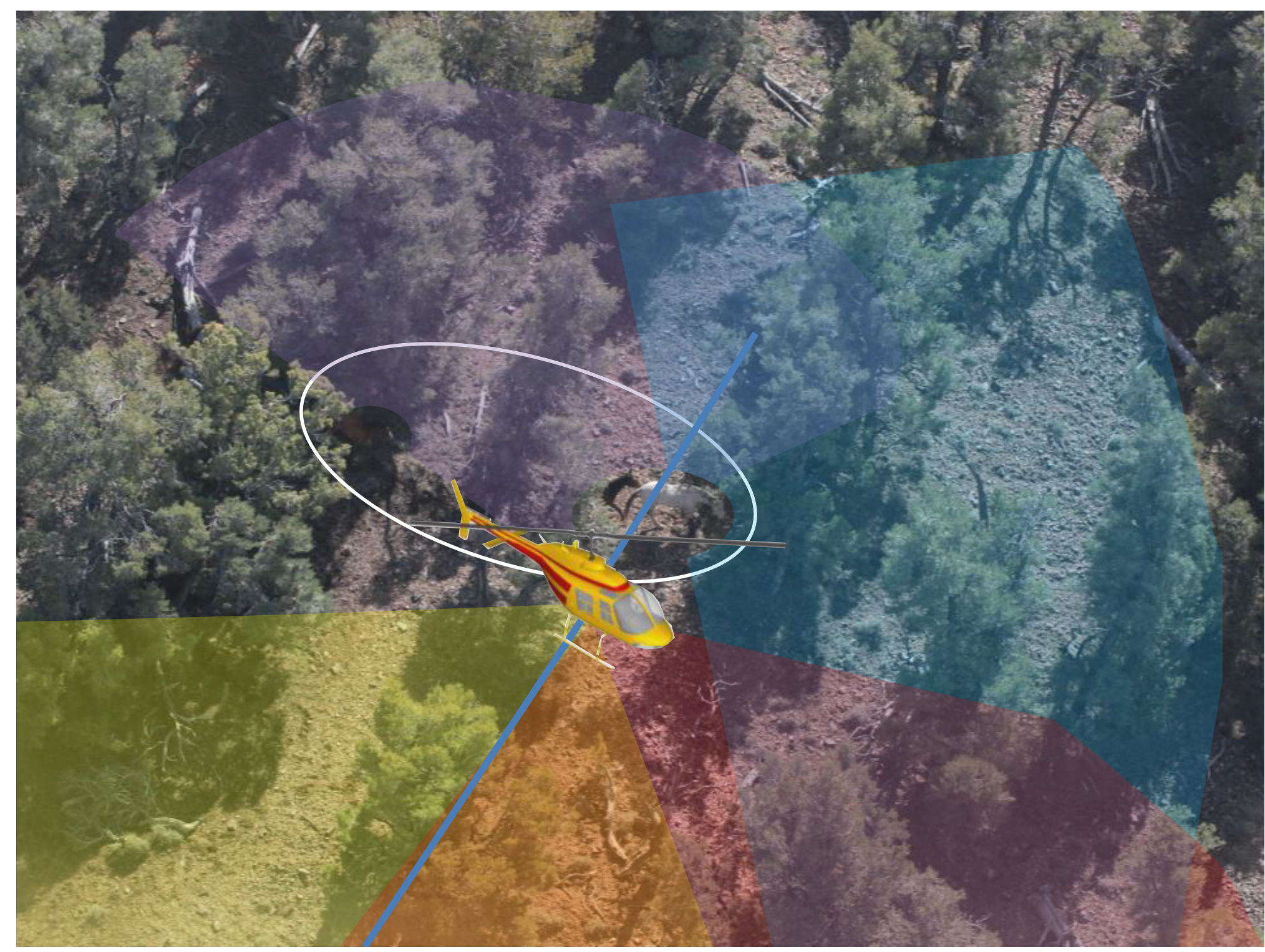

Figure 3. A diagram of a helicopter's continued flight past a horse group, circled in white. At this time, the horse group is abeam or past perpendicular of the helicopter flight path. At least one of the backseat observers has had a chance to detect this group. Any crew members who have seen this horse group should speak up now. The fields of view for the four aircrew members/observers are suggested by colored shading: yellow for the aircrew member/observer in the right back seat, red for the aircrew member/pilot in the right front seat, blue for the aircrew member/observer in the left front seat, and purple for the aircrew member/observer in the left back seat. The blue line suggests an axis that is perpendicular to the helicopter flight path. Photograph by Bureau of Land Management. 


\section{Counting and Photographing Horses after Detection is Announced}

A "detection group" is defined as any group of one or more horses that move together as part of the same visually identifiable social unit at the time of detection and get detected at more or less the same time in the process of observers searching for horses. The idea is that groups of horses (or burros) that are in observers' field of view together at the time of detection should be considered a detection group. If there is a large distance between two clusters of animals, such that they might get detected or missed separately, then it's more appropriate to consider them separate detection groups. Throughout this SOP, whenever we use the word "group," we are referring to "detection group."

A single detection group can be made up of one or more "bands." Typically, when specialists and range conservation officers observe horses on the ground, they keep track of the animals in small social bands, such as a stallion and associated harem and young, or a particular bachelor band. For the purposes of estimating population size with the double-observer method, what matters is the detection groups. This is because we know that group size is very important to detection probabilities, so if several bands are together in a single detection group, they are collectively more likely to be detected than if those bands were separate. For specialists that also want to keep records on the smaller bands within large detection groups, that is possible on the datasheet. We show how to record those data below in the "Defining Sighting Covariate Categories" section under Waypoint and Total Count.

After detection, the helicopter should be flown to maximize the chances of fully counting the horse group without causing undue stress to the animals. When the crew is counting group size and composition, the pilot should not fly directly over a large group, as the horses may scatter. Be sure to communicate this to the pilot. If horses bunch up or form a line after the helicopter hovers nearby, this is the best time to get a complete count and a photo if necessary. A hand tally counter may be useful for larger groups.

Survey crew members should decide before take-off what strategy will be used for accurate composition counts in large groups. One observer should photograph large herds while another observer obtains a preliminary count. In relatively open habitats, the best strategy may be to orbit the group so that the front seat observer and one other observer have the best view of the group. One observer may count adults, while the other counts foals. The pilot's attention should always remain on safe flight. The backseat observer who is not facing the horse group still plays an important role looking for additional horses outside the aircraft's orbit, and for flight hazards.

When horse groups are in tall timber, the most complete counts may come when the horses are visible to the pilot. If the horse group is first seen on the front seat observer's side, after an initial count of horses, the pilot should then fly so that the horse group is on the pilot's side. Observers on the side of the helicopter opposite the group should continue to search for other horses, maintain situational awareness, and watch for safety hazards. If the horses are already moving into heavier canopy cover, any observer with the best view of the horse group should ask the pilot to try to hold the position if it is safe to do so, and that observer should get as complete a count as possible. It may also be worthwhile to make one more orbit of the immediate area to look for more horses that become visible.

\section{Recording Location}

Horse group locations are important for long-term analysis of spatial distribution. Crews use two ways to record horse group locations: GPS waypoints and time. Most importantly, the location is marked directly with a GPS waypoint or given a waypoint number on a GPS-enabled laptop/tablet computer. As a backup, the time to the nearest minute is recorded when the helicopter is closest to each horse group sighted so that the group's approximate location can be mapped later, based on the GPSrecorded flight path. 


\section{Defining Sighting Covariate Categories}

When a detection group is spotted, observers record sighting covariates, which are attributes of that horse group that may influence their detection probability. These attributes are called "covariates." All the covariates must be recorded for each detection group observed, even if the detection protocol was not followed. It is important that all observers collect sighting covariate data in a uniform way across many HMAs and over many years. The things to record about every detection group are:

- Waypoint: A GPS-marked number that refers to the location.

- Species: Horse or burro. Other species can also be recorded during surveys.

- Total Count: the total number of animals in the detection group, recorded as adults and foals separately.

- Activity: whether the group was moving or still when first seen.

- Topographic class: whether there is rugged terrain near the group, that could hide a horse.

- Visual Field type: describing the field of view in a 10-m radius around the animals as open, broken, or trees.

- \% Concealing Vegetation: an estimate of the amount of vegetation in a 10-m radius around the group that could conceal a horse.

- Distance: Perpendicular distance from the group to the flight transect line.

- \% of Ground snow-covered: Percent of the ground and shrubs in a 10-m radius around the group that is snow covered.

Only the one 'data recorder' crew member records the covariates on paper, using the set of rules described here. There are many possible ways to define how a variable is recorded, but for consistency across time and space, all staff in the BLM WHB program (and in other agencies using these SOPs) should use the definitions below.

Throughout this SOP, a "default" value for a covariate is the one that will be entered in the data spreadsheet for analysis if no other value is circled on paper. The data recorder needs to specify such defaults, ensure that all covariate values are still recorded for each group, and note if conditions change such that the default no longer applies (for example, clouds move in and change light conditions).

\section{Waypoint}

Use a GPS unit to mark a waypoint for each detection group. The waypoint number will identify the detection group in all the analyses, so it is important for each group to have its own number. Each detection group gets its own waypoint, and its own row of data on the Survey Datasheet. It sometimes happens that two or more detection groups are seen from the same place on the helicopter flight line. If this happens, put a lower-case letter after the waypoint number, to distinguish each group. For example, imagine flying over a rise, and seeing one group off to the left, and a separate detection group off to the right. Mark one waypoint number (imagine it's \#57) and call those two detection groups 57a and 57b. When entered on the data spreadsheet, these will be two separate detection groups with waypoints $57 \mathrm{a}$ and $57 \mathrm{~b}$.

If separate bands that are all part of the same detection group are being recorded, they should all get the same waypoint number. To keep track of separate bands, record the waypoint number and a band number. For example, if there are three bands in the single detection group that gets waypoint \#68, it is okay to give each band its own line of data on the Survey Datasheet, and those three bands get numbered as $68 \mathrm{~B} 1,68 \mathrm{~B} 2$, and $68 \mathrm{~B} 3$. If this is done, just record the adults and foals that are in band 1 on the first line, along with the rest of the covariates for all the horses that make up the waypoint 68 detection group; then for band 2 and band 3 just record the number of adults and foals in those separate bands. When entered on the data spreadsheet, these will be all be recorded as just one detection group and waypoint 68 .

\section{Time (hh:mm)}

Record the time (24-hour clock) when the helicopter was closest to the group, to the nearest minute, for two reasons. First, time is used to identify photos that were taken of that group. Second, time can be used along with the flight line GPS log to find the group's location, if there is a problem with the GPS unit that was used for waypoint marking. 


\section{Species}

The datasheet is mainly for horse and burro group observations. Circle one of those two choices in the Species column of the Survey Datasheet. If an area has only one species (only horses, or only burros) is present, it is not necessary to circle anything here when that species is sighted, so long as that is noted on the Flight Information Form, or page 1 of the Survey Datasheets, that this is the "default" species for all observations.

It is not required to record observations of other species (that is, deer, elk, bears, bighorn sheep, eagles, coyotes, or any other wildlife species). Most districts find that searching for and recording those species distracts from the search for horses and burros. If other species are to be recorded, use rows on the Survey Datasheet. Only circle "Other" if other species that are not horses or burros are seen, but very clearly note what species it is in the comments section. Record the time the other species was seen (hours: minutes [hh:mm] format) and the number of animals in the other species group. For "other species" observations, it is not necessary to record who saw the group, or any of the sighting covariates.

\section{Total Count}

This is recorded for each detection group in two columns: the number of adults, and the number of foals. The total group size will be the sum of these two numbers. Total Count for adults and for foals on the datasheet may change after comparison with photographs taken during the survey.

If there is a detection group with more than one band in the group, record the number of adults and foals in each band, as noted above under Waypoint. Recording separate bands is not necessary from the analysis perspective. In fact, be very careful to record all the horses in the detection group, but also not to record them twice. If recording bands separately, it is important that those bands get associated as being part of the same detection group (in the example above, there are three lines of data; one for each band, numbered $68 \mathrm{~B} 1,68 \mathrm{~B} 2$, and $68 \mathrm{~B} 3$ ). When the time comes to enter the number of adults and of foals for the entire detection group for the data entry spreadsheet, add up the number of adults that were recorded separately for the bands that made up the detection group, and similarly add up the number of foals.

\section{Detected By}

The record of which observers independently detected the horse group is the key data source for estimating detection probabilities for horse groups, so this information is critically important to record. Detection probability estimates lead to the overall estimate of the number of horses in the surveyed area. "Independent detection" means that the observer saw some part of the detection group before anyone spoke up about the group or motioned to indicate about the group. Circle the observers that detected the horse group independently. The abbreviations on the datasheet are $L F$ (left front seat), $R F$ (right front seat), $L B$ (left back seat) and $R B$ (right back seat). If the pilot detected the horse group independently, then be sure to circle the seat position for the pilot. Sometimes, pilots forget to mention this unless they are asked.

\section{Side of Ship}

Record which side of the aircraft the horse group was on, so that it is clear which back seat observers had a chance to observe the group. $L=$ Left of aircraft; $R=$ Right of aircraft; $B=$ both sides of ship, or Center only (fig. 4). Both has a specific meaning; this means that the group spanned both sides of the aircraft, so it was detectable from both rear seats. Both can also mean a group that runs from one side of the aircraft to the other and therefore available to be detected by observers on both sides of the aircraft. Center only has a very different meaning; it means that the group was never visible to the rear seat observers because it was always immediately in front of or under the aircraft. Rear seat observers should always look out the side of the ship, so they have effectively no chance to see a group that is positioned center only. To envision how wide the center only area is, picture that it is the area on the ground that is invisible to the backseat observers because it is between the skids in a helicopter. The area that is invisible to back seat observers in an airplane will depend somewhat on the make, model, and height AGL.

\section{Activity}

Choose either Move (animals were walking, running, or otherwise moving) or Still (animals were standing or lying down). The choice recorded is the one that best describes what animals first detected were doing at the time they were first detected. If a moving animal was the first animal detected, record Move even if the rest of the group was standing still. 


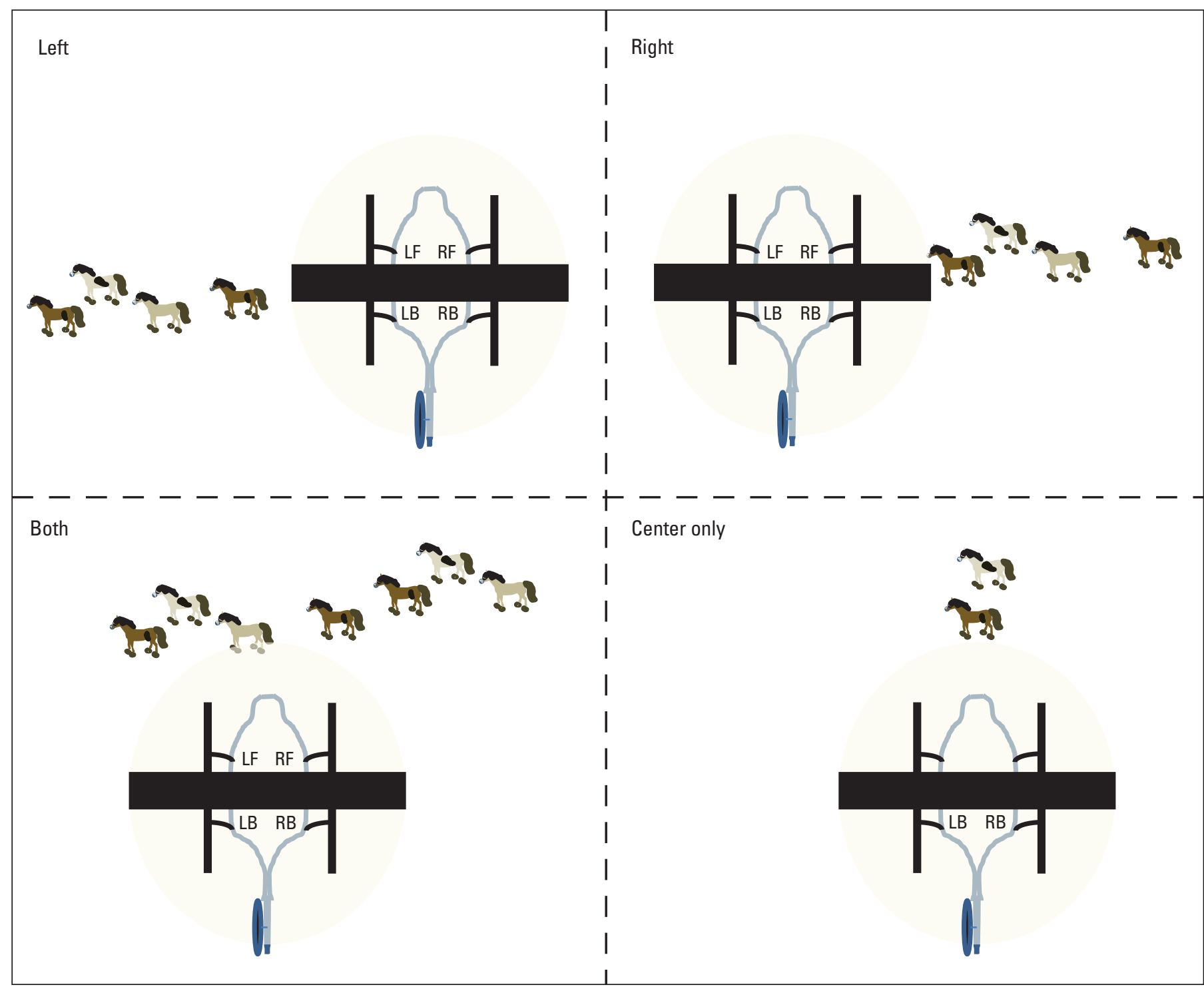

Figure 4. A diagram of the four choices for "Side of Ship" for aerial surveys. For "Left" or "Right", the horses were exclusively on one side of the helicopter and its flight path. For "Both", the horses were positioned in a way that they could have been detected by both backseat observers. For "center only," the horses were never available for detection by the backseat observers because the aircraft flew immediately over the animals; the animals appeared to be between the skids at all times until the aircraft passed over them.

\section{Visual Field}

Select just one visual field category that describes the visual field in the patch where the majority of the horse group was located when the group was first seen. This covariate is meant to describe the visual field in terms of the quantity and height of vegetation that was breaking up the observers' views of the ground. Choose the visual field category that best describes the greatest proportion of an area defined by a 10-m buffer surrounding the entire horse group at the time it was first detected. For large groups, observers may first need to assess where the majority of the group was at the time when the group was first detected, then select the visual field category.

Visual field categories are Open ( 75 percent or more of the ground is covered by bare ground, grass, or vegetation that is low, that is, below horse hock height), Broken (the visual field is broken up by vegetation, with 25 percent or more of the ground is covered in vegetation that is from hock to horse-head height (fig. 5), and Tree ( 25 percent or more of the ground is covered by plants that are horse head height or taller (fig. 6). If there is both 25 percent or more of hock-to-head high plants and 25 percent or more of tree-height plants, choose Tree, because in that case the higher vegetation probably has a greater effect on the overall visual field than the lower vegetation. Choose only one type. 


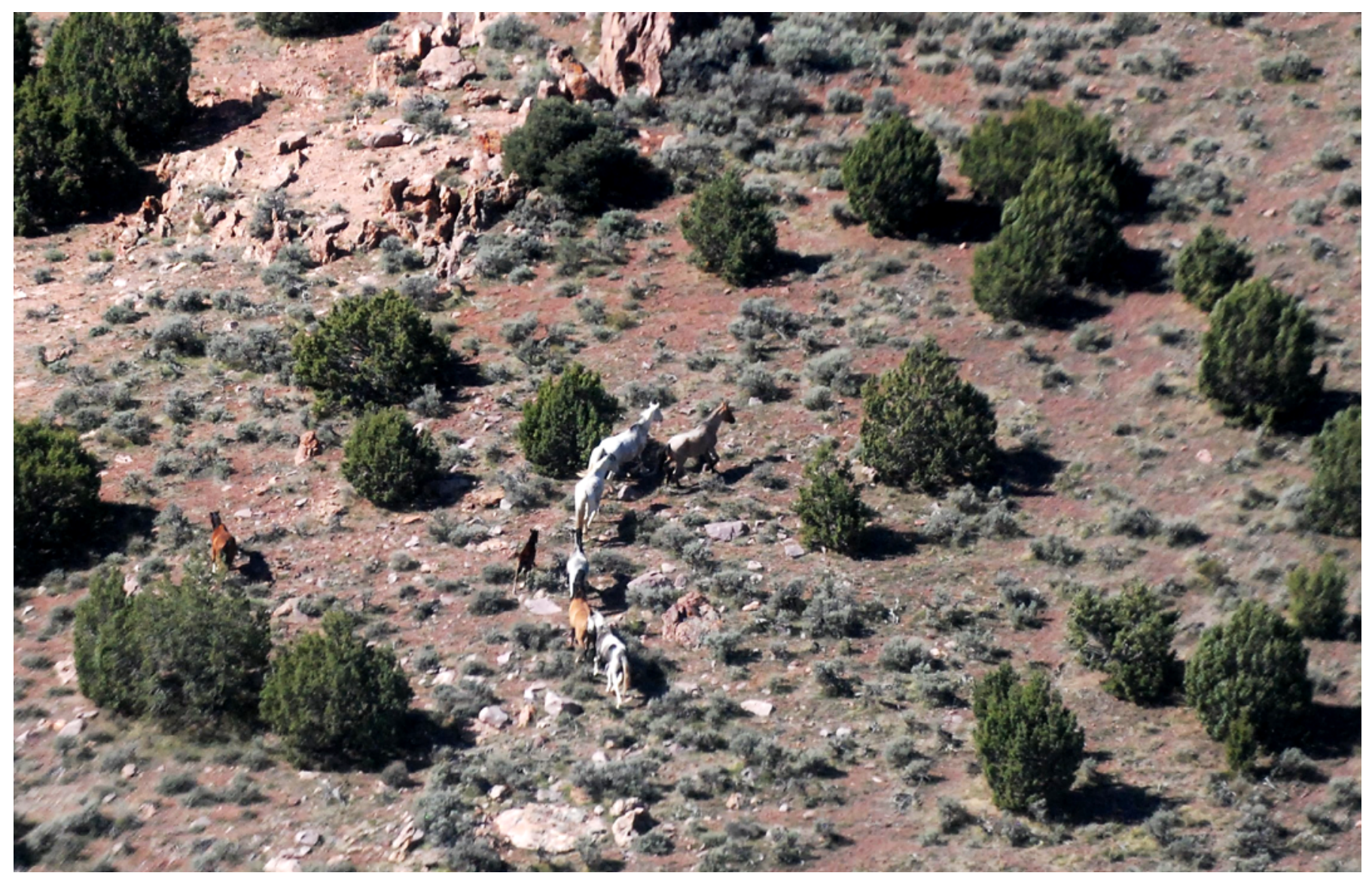

Figure 5. A photograph of horses in a "Broken" visual field type. Although there are trees, they occupy less than 25 percent of the ground space within the area of the group plus 10-meter (m) buffer. Photograph by Chad Hunter, Bureau of Land Management, 2012. 


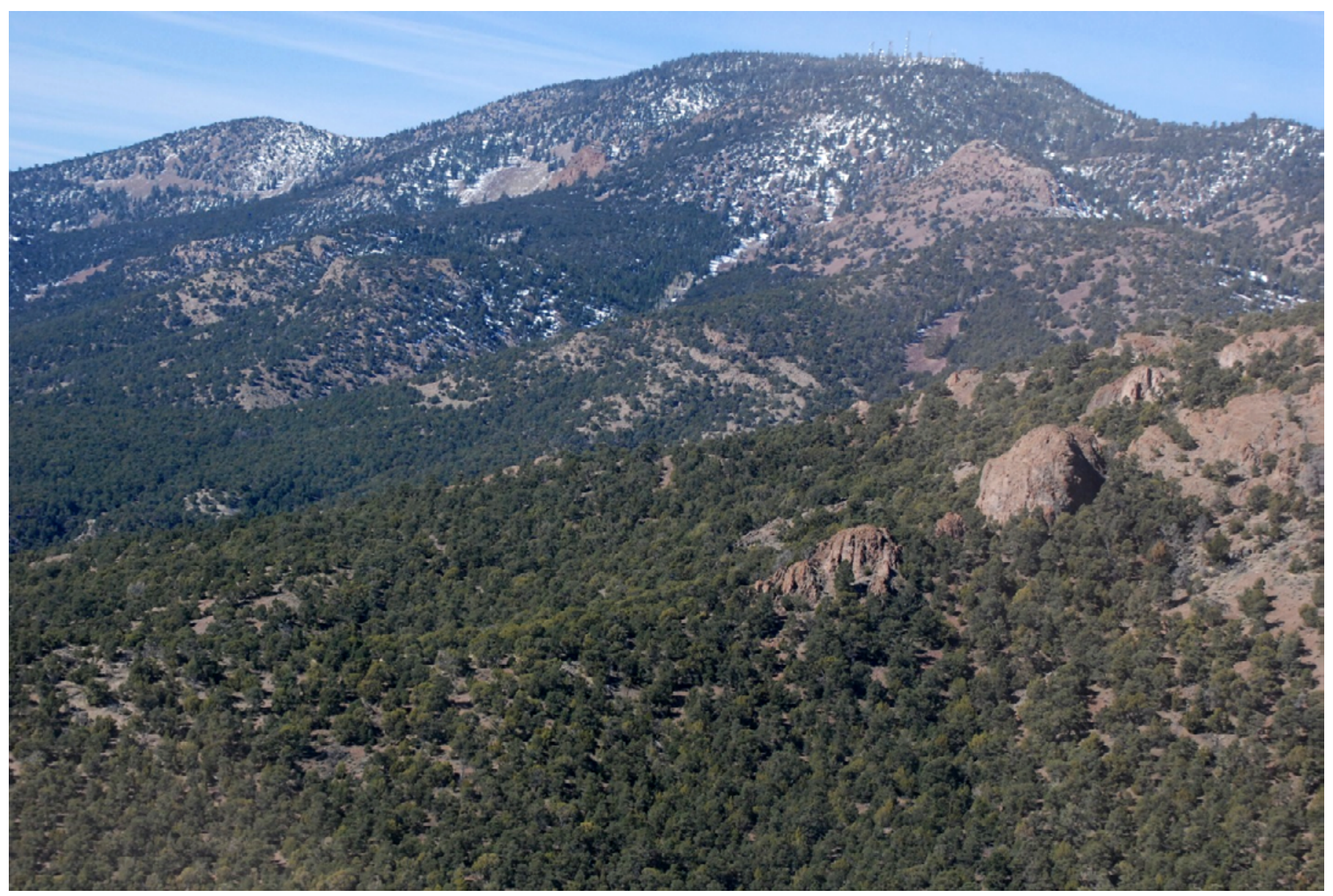

Figure 6. A photograph of a "Tree" visual field type. At the upper right are two topographic features that would make the topo class "Rugged" if a horse group were near them. Photograph by Chad Hunter, Bureau of Land Management, 2014. 


\section{Percent (\%) Concealing Vegetation}

Percent concealing vegetation is the observers' estimate of the percent cover of vegetation that has the potential to obstruct observers' view of horses (Unsworth and others, 1994), or burros. The key idea is to record only the vegetation that could conceal part of a horse (or burro) from hock to head height or taller. Record \% Concealing Vegetation to the nearest 10 percent $(0$, $10,20,30,40,50,60,70,80,90$, or 100). Decide which percentage best describes the concealing vegetation, evaluated over the area of the whole detection group, plus $10 \mathrm{~m}$ ( $\sim 10$ yards $)$ in all directions. This percentage includes both the effects of trees and shrubs. If it helps, try to imagine the average percentage of concealing vegetation it would be if the group could be seen from all sides. The question to answer is, within that oval volume that contains the whole group plus $10 \mathrm{~m}$, how much tree or shrub vegetation is there that could conceal a horse?

The percent concealing vegetation is defined for a 10-m buffer surrounding the entire detection group (figs. 7 and 8), including those first seen as well as other horses in the group that are seen after closer examination; the latter are often under denser cover.
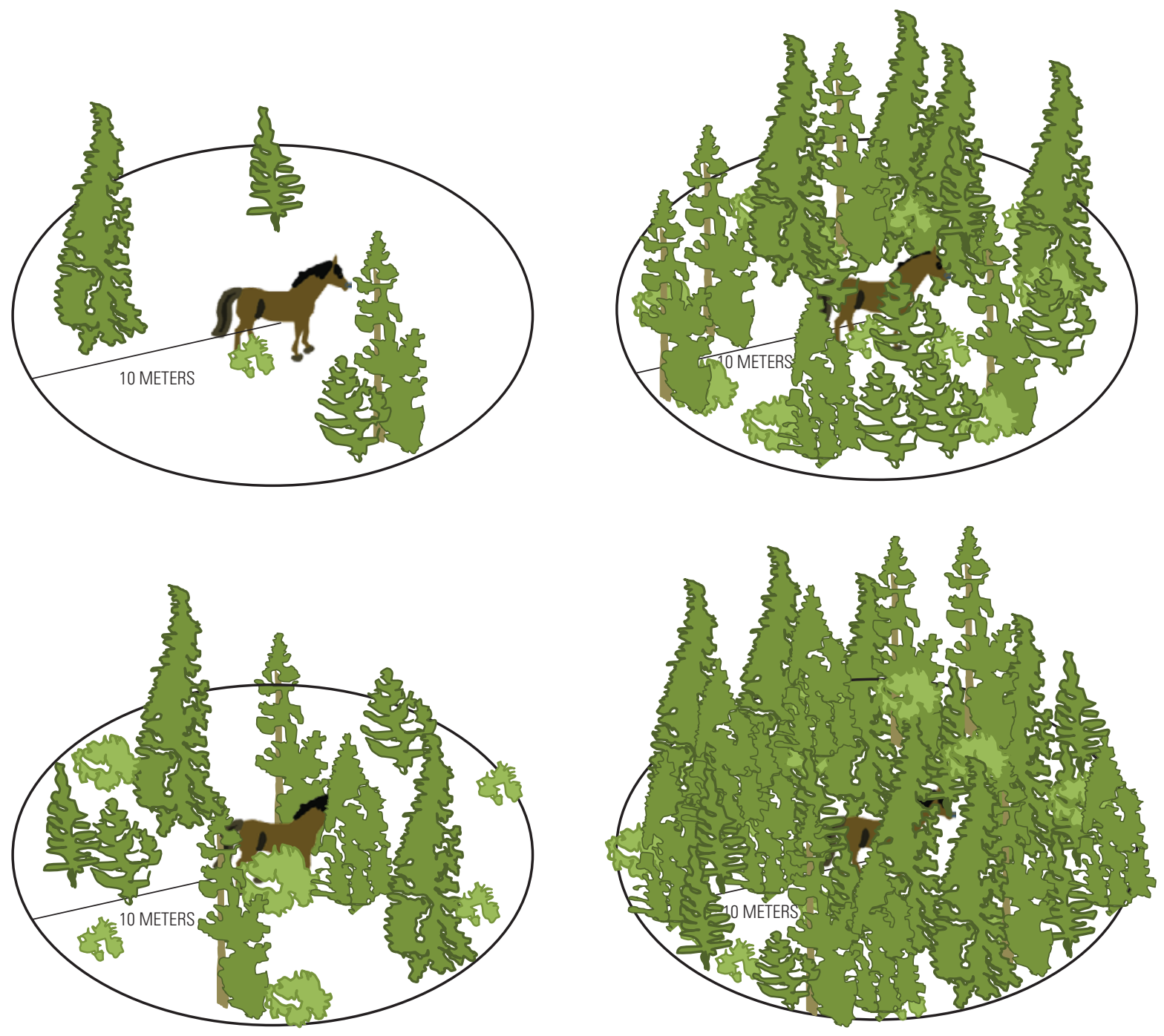

Figure 7. A diagram of four examples of percent concealing vegetation, illustrated for a single horse. The value of "percent concealing vegetation" for the 10 meter $(\mathrm{m})$ radius area around this horse is chosen from categories defined in the text. The value for the horse in these examples is $\sim 10$ percent (upper left); 30 percent (lower left); $\sim 60$ percent (upper right); and $\sim 90$ percent (lower right). Illustrations are based on Unsworth and others (1994). 

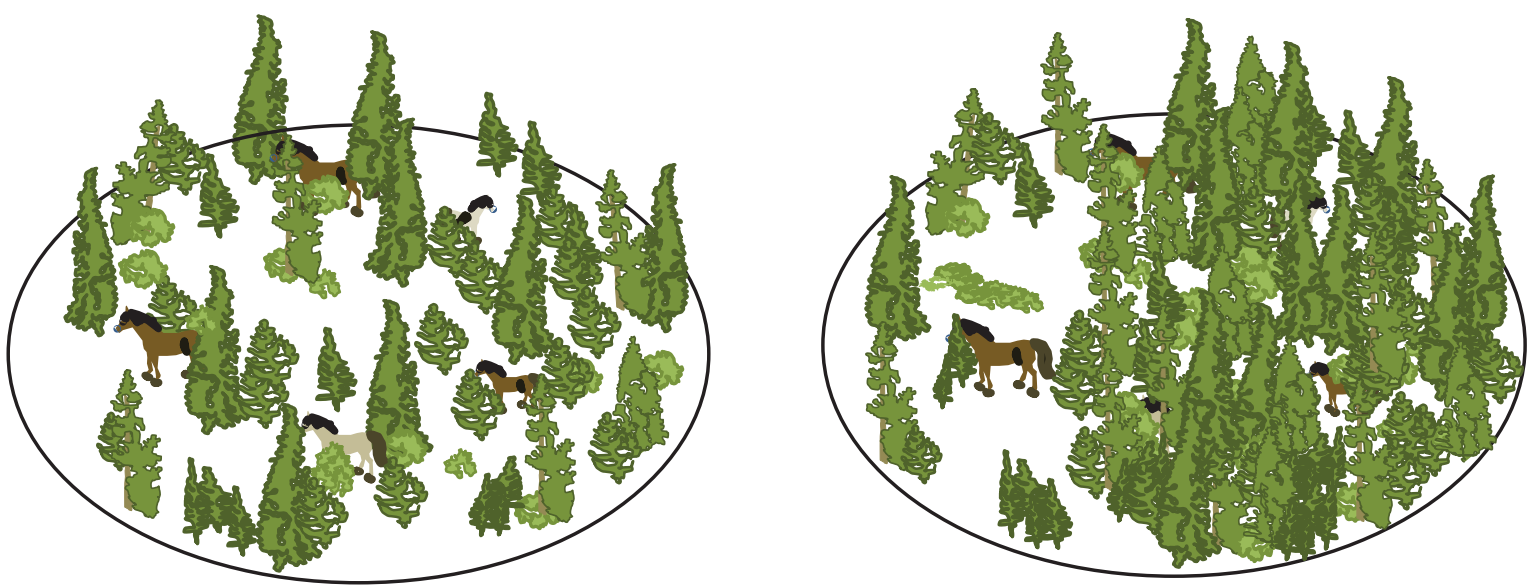

Figure 8. A diagram of two examples of percent concealing vegetation, each with a detection group of five horses. The percent concealing vegetation is evaluated for the area occupied by the whole horse group. Determine where the whole group was when first detected, then evaluate the percent concealing vegetation for that area plus 10 meters $(\mathrm{m})$. The percent concealing vegetation values would have a value of $\sim 20$ percent (left) and $\sim 60$ percent (right). Illustrations are based on Unsworth and others (1994).

\section{Distance}

Record the approximate closest horizontal distance in miles from the where the horse group was when it was first detected to the transect line being flown when they were first detected. For horse surveys, circle one category: $<1 / 4$ mile $(<400 \mathrm{~m})$, $1 / 4-1 / 2$ mile $(400-800 \mathrm{~m}), 1 / 2-1$ mile $(800-1,600 \mathrm{~m})$, or $>1$ mile $(>1,600 \mathrm{~m})$. For burro surveys, the flight lines are usually closer together, so circle one of these categories on the burro survey datasheet: $<100 \mathrm{~m}$ ( $<110$ yards), 100-200 m (110-220 yards), 200-300 m (220-330 yards), or 300-400 m (330-440 yards), or if the distance to the burro group was more than $400 \mathrm{~m}$ (440 yards, $1 / 4$ mile) then note the approximate distance in the Comments box. When thinking about what was the "survey line," don't include the part of the flight path when flown closer in order to count the group. There are specific notes about what distance to record in rare situations in the section "More About What Distance to Record."

\section{Light Level}

Circle the one light condition that describes the area of the detection group plus a $10 \mathrm{~m}$ buffer. Flat means that the light level was flat, that the entire area between the aircraft and the horse group was in shadow of clouds or low light, with no distinct shadows on the ground. $\mathrm{Hi}$ means high contrast, that there was bright light and shadows at the horse group. If the horse or burro group is standing in or near some shadows cast by trees or vegetation, record Hi. Shade means that the horse group was in the shade of a cloud, cliff, or rock, but that there were sunny places between the aircraft and the horse; the horses were in the dark, but there were areas on the ground that were brightly lit between the horses and the aircraft. Shade would be a possibility on days with scattered clouds casting dark shadows, and where there is some bright sunlight between the aircraft and the horses. Shade would also be the right choice if the group was in the shade of a large rock or cliff, but there was some brightly lit area between the observers and that shadow. If the horses and the observers are under the shadow of the same cloud with no bright spots in between, record Flat. In trees, only record Shade if the shadow itself affects detection - for example a burro in 30 percent tree cover hiding in the shade of a tree on a hot sunny day, compared to a burro in the same 30 percent tree cover standing in the sun.

\section{Topo Class}

This covariate describes whether topography, terrain features, or large boulders could hide the horse group. The choices are Smooth (open terrain with little to no rocks that could hide a horse) or Rugged (rugged terrain with rocks or topography tall enough to hide a horse or burro). The metric to use is whether any horses in the group were ever obscured by topography as they are approached. If any were ever obscured, it's Rugged. If the whole group can be seen the entire time while the helicopter approached, it's Smooth. 


\section{Percent (\%) of Ground Snow-Covered}

This covariate describes the percent of ground within $10 \mathrm{~m}$ of the entire horse or burro group that is covered in snow, to the nearest 10 percent (fig. 9). Include snow that is on low vegetation, such as grasses and shrubs. Do not include snow that is on trees.

\section{Protocol OK?}

The simultaneous double-observer protocol is what was described above: each observer has an independent chance to detect the horse group. So long as that method was followed for a given group, the choice for this column of the datasheet should be $O K$. In general, this means that the pilot, front seat observer, and back seat observer on the same side as the horse group all had a fair opportunity to either detect or not detect that group. Specifically, no one spoke up, motioned, or otherwise clued in back seat observers before they had a chance to spot the group. If back seat observers are clued in, simply circle no on the Survey Datasheet. $O K$ is in bold font because it is the default choice, and the recorder only needs to circle no if the protocol was not followed. Groups where protocol was not followed will be used to estimate abundance but will not be used to fit detection models.

Count every horse group that can be seen but make every effort to follow the protocol for all groups observed. If the doubleobserver protocol is not followed, circle no and make a note in the comments section explaining why it was not followed. It is always better to call out a group early and go "off protocol" than to lose the opportunity to count and identify that group. For example, if the front seat observer sees horses running into the trees and that they will be impossible to find if the pilot continues to fly straight long enough for the backseat observer to get abeam, then the front seat observer can tell the pilot to go off the pre-planned flight line and head off the group, for counting. If this happens, let the data recorder know that group will be "off protocol."

There is another common reason an observation might be off protocol. If a first group has been announced, the aircraft is orbiting around that first group, and most of the crew has their attention on counting that first group, sometimes one observer will notice a second group to the outside of the orbit's circle (see fig. 13). Given that the rest of the crew was focused on that first group, they did not have a fair chance to detect the second group. In this situation, record that the protocol was not followed for the second group (Protocol OK? = no).

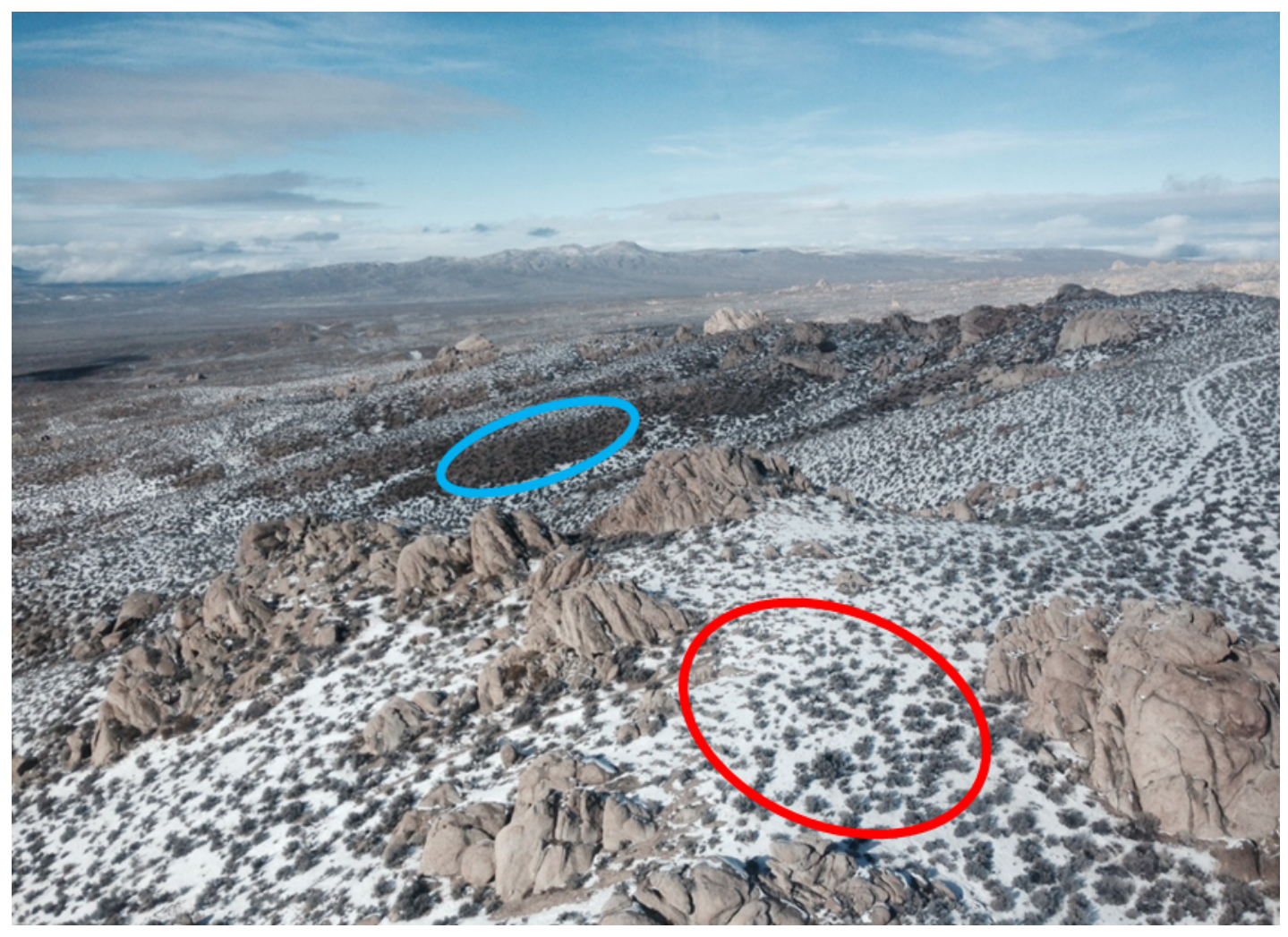

Figure 9. An image with two examples of percent snow cover. The area in the red oval in the foreground has approximately 60 percent snow cover, while the area in the blue oval has approximately 20 percent snow cover. Photography from Bruce Thompson, Bureau of Land Management, 2015. 


\section{Comments}

It is optional to record comments about any detection group. It can be very useful to record notable colors of horses, to distinguish groups and prevent duplicates (double-counts). It is easier to distinguish horse groups that are close to a previouslymarked waypoint if the data recorder consistently records notable colors in each group. The comments area of the "Survey Datasheet for Horse Surveys" in appendix 3 includes several of the more common colors, with a line next to them. It is optional to use these. For example, the data recorder may choose to make a mark to indicate that a group had one or more such colored groups, may choose to record the number of each color of horses in each group, or may leave the area blank.

This is also the area of the datasheet to make any other notes about an observed group. If the comments box is too small for the notes, just write additional comments in the next line down below that group and make a mark or arrow to show that the comments are associated with that group.

\section{Data-Recording Shortcut-Default Values}

Default values are values that are so common on a survey that the data recorded does not need to take the time to circle the choice on every observed horse or burro group. Some covariates have default values in bold text, already printed on the survey datasheet: the $O K$ is in bold in the Protocol OK? column because we assume that the double-observer protocol will be followed for most observations, and Smooth because rugged is rare in most surveys. Other commonly used defaults are zero for percent concealing vegetation (if there are no trees) and zero for snow cover (if there's no snow). When using defaults, only circle choices when the default value is not correct.

The data recorder must clearly specify any default values on page 1 of the survey datasheets for each flight. Do not assume that people reading the datasheets will know what the default values are, or that the recorder will remember when looking at the datasheets months or years later. The data recorder must also be careful to record all covariates for each observed group-it is easy to forget to fill in a not-default covariate when it only shows up rarely.

\section{What to do When the Same Group is Detected Twice}

Each group of horses that is observed should only count once toward the estimate of the total number of animals. Therefore, any time that the same group is seen twice must be clearly identified as a repeat observation on the Survey Datasheet.

Occasionally the crew will detect a group, record its location and all covariates, then detect that same group again later in the survey. When traveling along each transect passing through an area, it is okay to make a new record showing that the previously-recorded group was detected, but be sure to note in the comments that it is a repeat observation of a group that was already seen, and note the waypoint for the observation when that group was first recorded. It is not required to record a group a second time; it is only optional. Why bother to record the same group more than once? Because each detection gives more information to the statistical analysis about detection probability. Clearly note on the Survey Datasheet if a group has already been recorded so that no horse or burro groups get "double-counted." And only record the group a second time if the detection really is independent - it is not an independent detection if the observers know where the group will be as they're approaching it.

\section{Photography}

Large ungulate groups seen from the air have a tendency to be undercounted (Cogan and Diefenbach, 1998). Detection groups of $\sim 20$ or more animals must be photographed with a high-definition digital camera (BLM, 2010). Observers may want to take photographs of any detection group with 10 or more animals, especially if they are bunched up. Photographs are used after the survey to compare group size and composition recorded on the datasheets to animals seen in the photo. Always synchronize the camera's clock with the GPS unit time before the flight so photographs are associated with the appropriate horse group observations based on the time the group was observed.

The photographer should try to photograph all members of the group in one frame, before they disperse. If that is not possible, the photographer should tell the data recorder how many horses of a group were not included in the photograph; that should be written on the Survey Datasheet in the comments area for that horse group.

In most cases, the photographer should be the front seat observer. In some cases, a backseat observer will have the better view. Generally, the data recorder should not be the photographer.

The optimal camera type (as of 2017) is a digital single lens reflex (SLR) camera with an image-stabilizing zoom lens. This should be in sports mode, with the image stabilizer and autofocus turned on. To frame the photograph, zoom manually, push down the shutter release button half way so that the camera automatically focuses, then push the button all the way to take the picture. Take multiple photos of the same group, then take a photo of your shoe to make a break in the photos between groups. A reasonable resolution initially, for classification is at least $1,600 \times 1,200$ pixels ( $\sim 2$ megapixels). 


\section{More about Recording Covariate Data, and Who Saw the Horse Group}

As soon as possible after each group of horses is observed, the data recorder should fill in values for the covariates. Recorded covariate values should be based on the consensus opinion of all the crew that saw the horse group (see the section "Defining Sighting Covariate Categories").

It is imperative to record exactly which observers detected the horse group independently, by circling up to four "who saw" choices in the Detected by column. The data recorder should ask to clarify who independently detected each group. Detections made after someone else pointed out a group are not independent; only observers who independently detected a group should be circled. After all covariates for the horse group have been recorded, the data recorder should read the covariate values out loud, as a final opportunity for other crew members to offer corrections.

\section{Special Circumstances that Will Undoubtedly be Faced}

There are a few circumstances in which the question arises about whether the protocol was followed ("Protocol OK" $=O K$ ) or not ("Protocol OK" = no). In all cases, be sure to record all covariates and the number of adults and foals (Total Count of Adults and Foals, Side of Ship, Activity, Visual Field, \% Concealing Vegetation, Distance, Light Level, Topo Class, and \% of Ground Snow-Covered) for every horse or burro group seen, whether the protocol was followed or not.

The first circumstance is when one detection group is observed, then a separate detection group is discovered while circling the first. Record that the protocol was followed if both front and back observers on the aircraft had an independent opportunity to see the new, separate horse group. Record that the protocol was not followed if, for example, the front observers' attention was all given to counting the group first detected, and the back observer noticed the new, separate group during the circle. Also, record that the protocol was not followed if, during the circle, the front observer called out that there is a new, separate detection group out there, but the back observer did not yet have a chance to independently observe that new group.

The other common circumstance is when, upon arrival at an area, there are several separate detection groups scattered around, and some are running away. Considering them separate detection groups means that they would not have been visible in the same glance, or that there is enough distance between them that they could have been seen or missed as separate units. How should the recording and counting of these several detection groups be done? In this case, it is up to the front observer to use judgment. A key idea in these surveys is that none of the groups should go uncounted or escape into cover before they can be recorded, even if it means going "off-protocol." Less importantly, it is beneficial for analysis if the backseat observers have independent opportunities to detect as many of the groups as possible, following the protocol. If the front seat observer thinks that the horse groups in the area are staying put, then continue on your pre-planned flight line through all the groups, record each separate group with its own line of data, and then come back to do any closer circling to get more accurate counts of each group.

However, if, the front seat observer (or pilot) judges that one or more of the groups out in front of the aircraft are going to get into cover or out of sight before the back seat observers will have a chance to detect the groups, then the front seat observer should tell the pilot to change course if needed to get an accurate count of the escaping groups as soon as possible. The data should be recorded to show that the protocol was not followed for those groups, but it is always better to record a horse group even if the protocol is not followed, than to let that group get away uncounted.

The other important point about how to record data when there are multiple scattered groups is that each separate detection group should be recorded as its own line of data. As noted earlier, a "detection group" is defined as any group of one or more horses that move together as part of the same visually identifiable social unit at the time of detection, and which get detected at more or less the same time in the process of searching for horses. In the hypothetical case where there are several detection groups scattered across a large valley, record each detection group separately. If it is clear that the several bands are one social unit with no large break between them, or they all line out together when moving, then record them as one detection group (it is optional to record one line per "band" within a single, larger, detection group (see Waypoint, and Group Size). Always record all the covariates for each separate group of animals.

All observers should search the entire field of view, from right under the aircraft, out to the far distance. Be aware of the approximate location of the next parallel transect line that will be traveled. Share your detections of every horse group that is between the current position and the next transect line over. If there is a horse group beyond the next transect line, that is a special circumstance. If a group beyond the next survey line is standing still, and you will soon return to the transect near that horse group, then it may be better to wait to announce the group is there until the rest of the crew members have had their fair opportunity to either detect or not detect that horse group. But if a far-away horse group is moving away, or the group may not be there when the helicopter returns, then it is always better to tell the rest of the crew about that group, and to go count it right away. As long as both the front seat and back seat observers had a chance to see the group, groups that are farther away than the next transect should be marked as "Protocol OK" = yes. Record every group that gets detected, even if it was recorded before but be sure to note in the comments any group that has already been recorded. For example, if a group is recorded at waypoint 27, then that same group is seen again on a later transect, write "Repeat of group 27" in the comments for the observation that is a repeat of that same group. 


\section{More About What Distance to Record}

Observers should be sure to search for animals anywhere from right underneath the aircraft out to the next transect line, but also to expend some effort searching out to the far distance. Try to be aware of where, approximately, the upcoming survey lines will be on the landscape. The closest distance from the group to the current transect is on the perpendicular line from the horse group to that transect (fig. 10). So, if a front seat observer sees a group for 2 miles directly in front, but the horses are still standing in the same place when the aircraft passes less than $1 / 4$ mile from the group, record " $<1 / 4$ " mile.

It is also common to detect a horse group that is closer to the next transect line than the one the observers are currently on. Record the distance from where the horse group was when it was first detected, to the transect line the observers were on when the group was detected (fig. 11). Sometimes, the horse group will start to run before that next transect line is flown; even in that case, record the distance from where the group was when first detected to the transect line the aircraft was on when the group was first detected (fig. 12). Figure 13 shows what distance to record when new horse groups are detected while circling a first group, and figure 14 shows what distance to record when you have to divert from the current transect to record a distant group that is running away.

When thinking about what was a "survey line," or "your circle," don't include the part of the flight path when flown closer to group 2 or 3 in order to go count those groups and record covariates for those groups. Use the closest distance the observers came to the group, other than the flight path followed to get a closer look at that group.

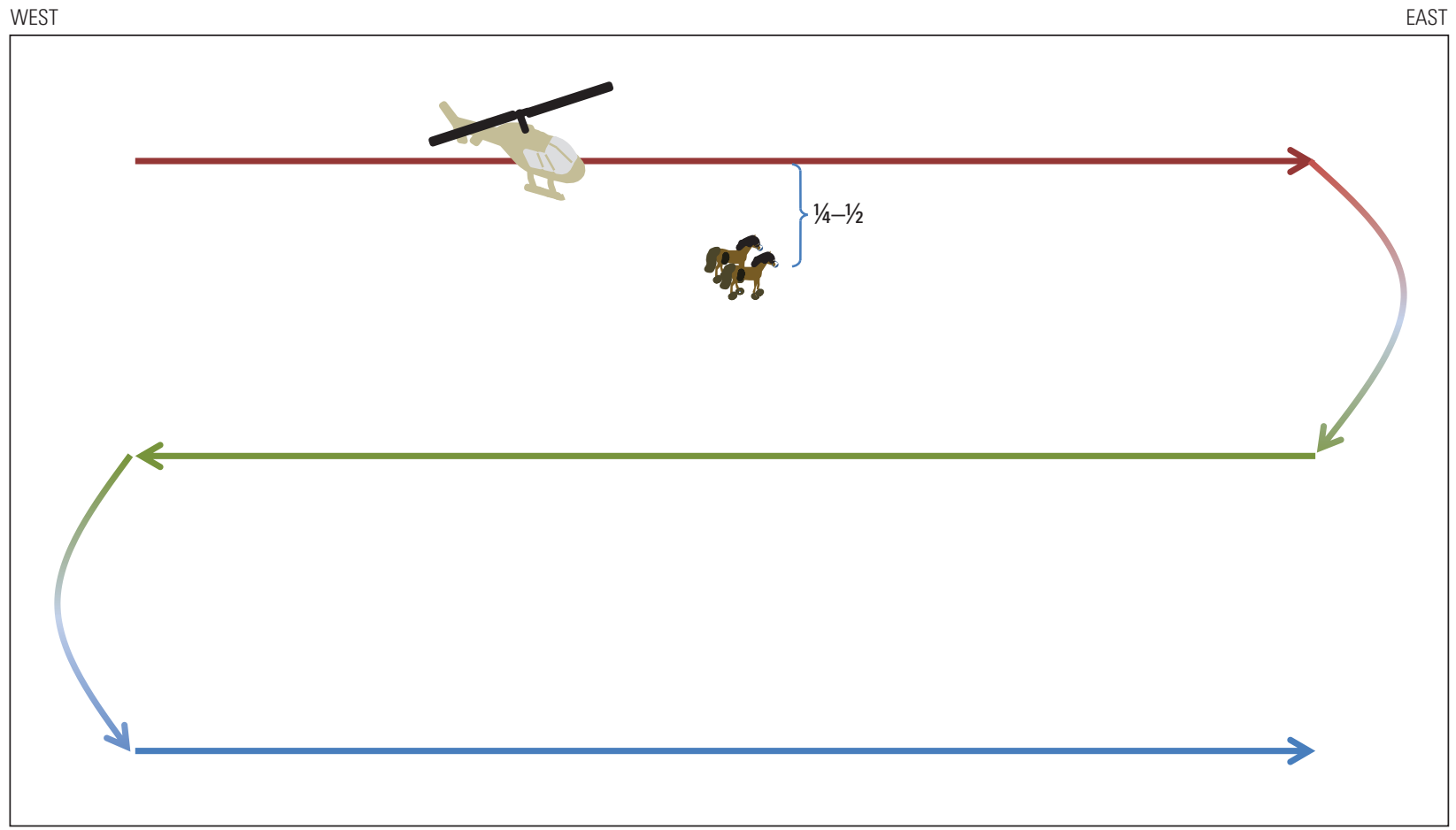

Figure 10. In this diagram of a scenario, the helicopter's planned survey lines are red, then green, then blue, and there is 1 mile between lines. The horse group is detected while the survey crew is on the red line. The distance from the transect line to record for this horse group is between $1 / 4-1 / 2$ mile. 


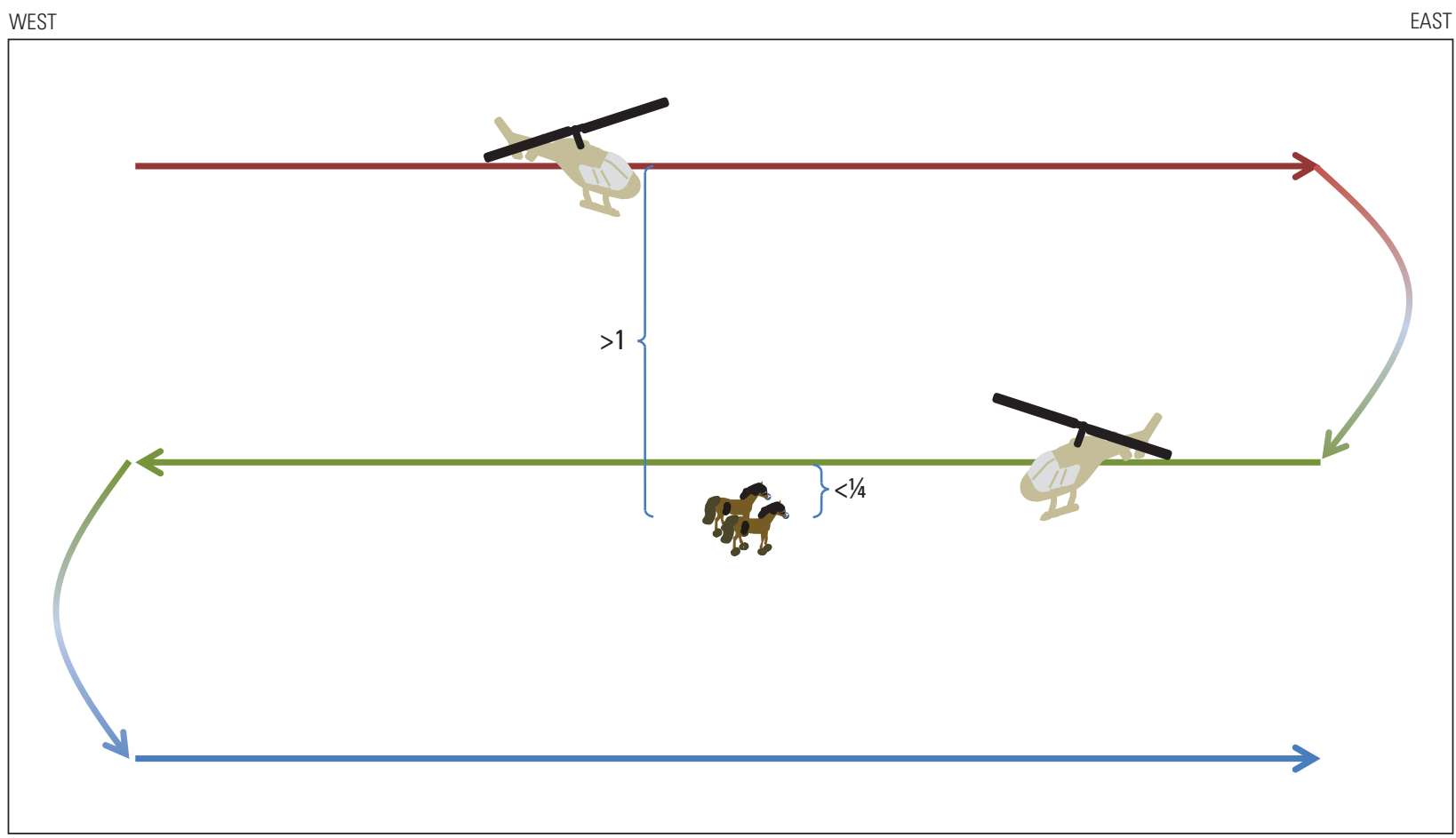

Figure 11. In the diagram of this example, also with 1-mile transect spacing, the horses are first seen more than 1 mile from the red transect line, going east, and were also seen less than $1 / 4$ mile from the green transect, going west. The first time the group is detected, the distance was just over a mile, so record " $>1$ " mile as the distance. The second time the group is recorded, be sure to make clear in "comments" that the second observation is a repeat detection of the same group. For that second observation of the group, record a distance of " $<1 / 4$ " mile. The helicopter's planned path along the transect lines are red, then green, then blue. 


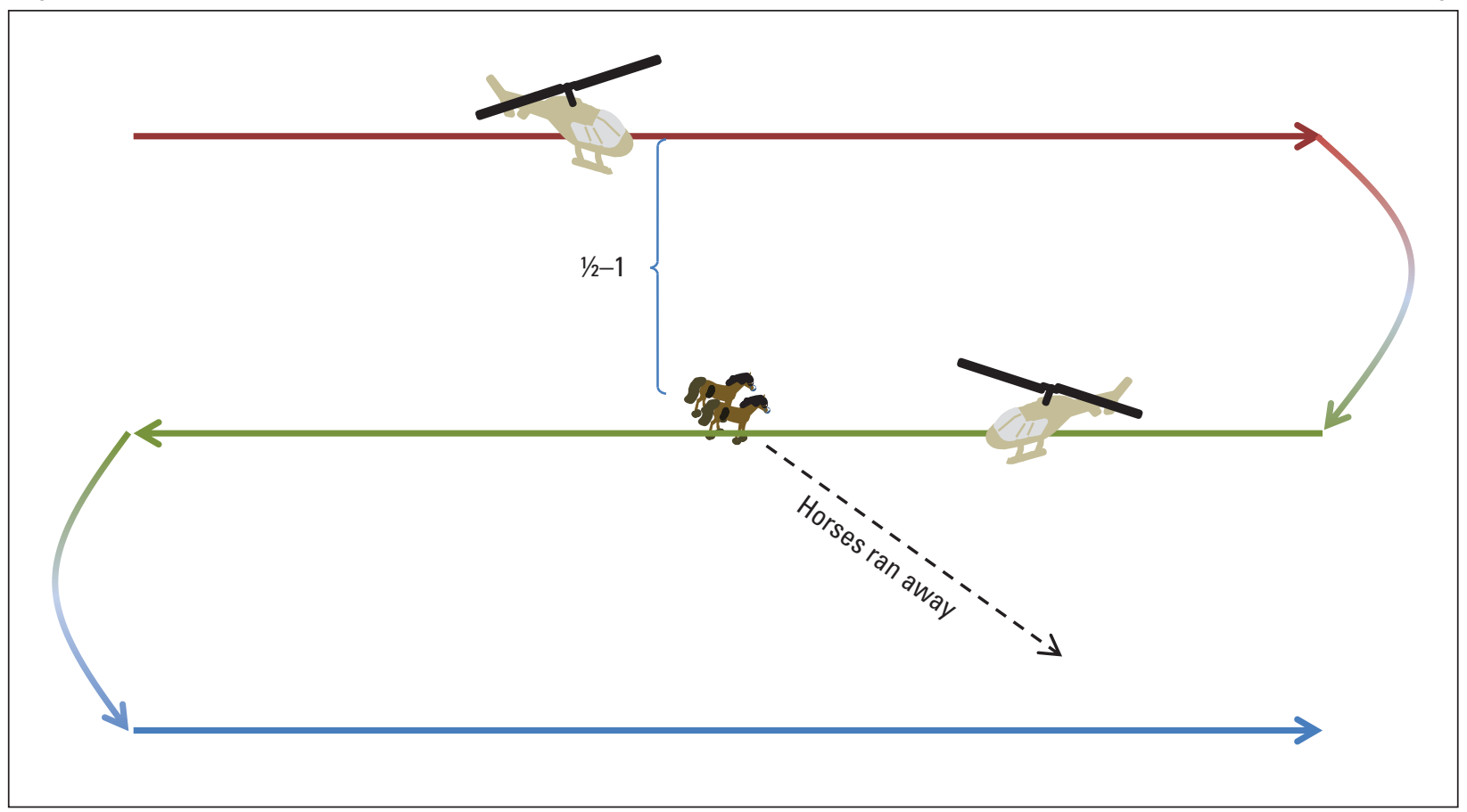

Figure 12. In the diagram of this situation, again with 1-mile transect spacing, a horse group is slightly less than one transect away, when first detected from the red line. The aircraft stays on planned transect lines. Get a good count from the red transect line. Record the closest distance from where the horse group was when it was first detected to the transect line the helicopter was on when the crew detected the group: $1 / 2-1$ " mile. The horses have moved away from the green line by the time the helicopter comes back to where they were, so do not record the group again. The helicopter's planned path along the transect lines are red, then green, then blue. 
WEST

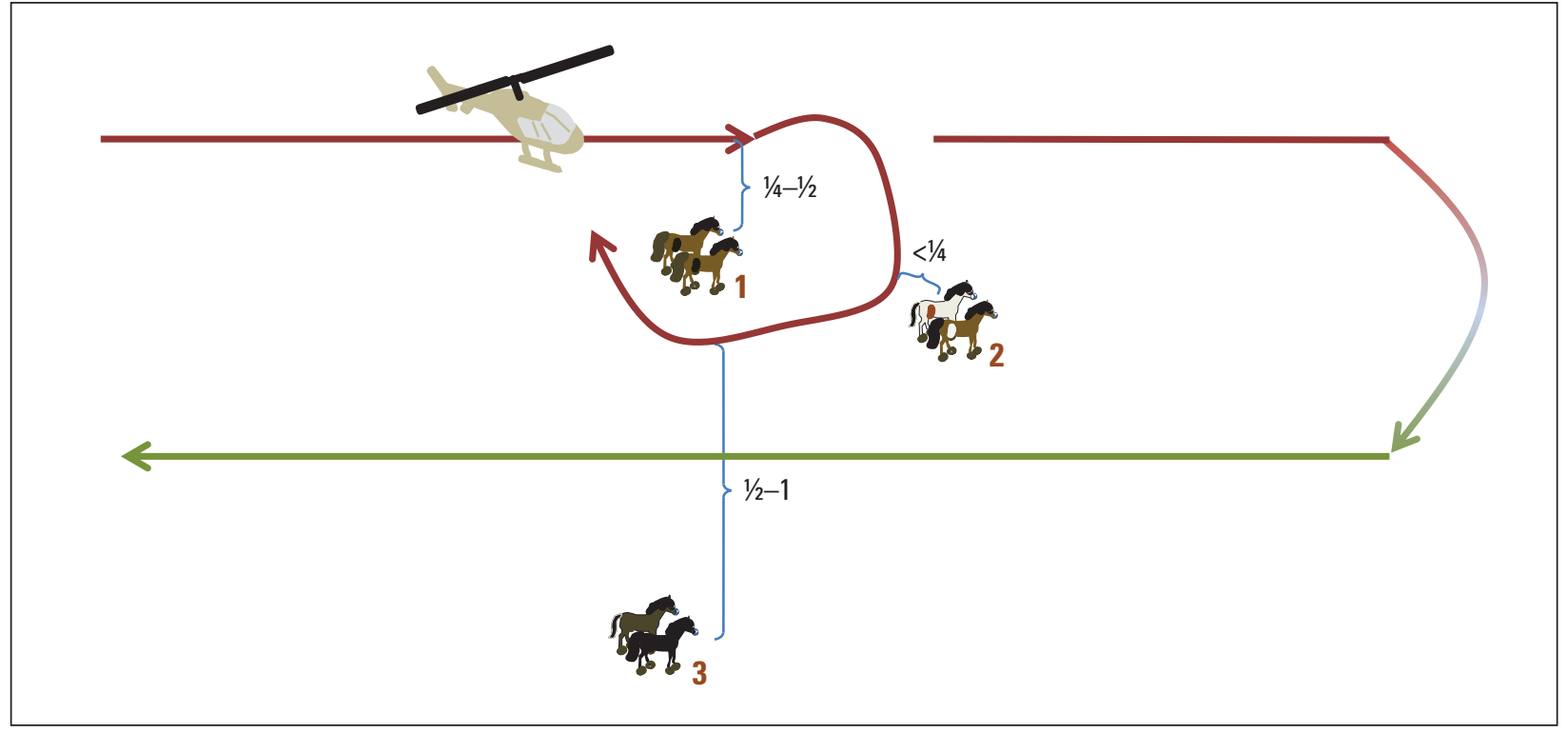

Figure 13. In the diagram of this situation, extra groups are seen while circling to get a closer look at the first group that is detected. For groups seen from a transect line, record the closest distance from where the horse group was when it was first detected to the transect line the observers were on when the group was first detected. For groups seen when the crew was circling around another group, record the closest distance from where the horse group was when it was first detected to the circle the crew was on when the horse group was first detected. In this example, imagine that horse group 1 was detected according to protocol (protocol $0 K),<1 / 4$ mile from the red transect, on the right side. Then, while the right front and right back observers were focused on group 1, the left back observer saw group 2 (protocol 0K? = "no," because not all observers had an opportunity to detect). Also imagine that while circling, the right back observer saw group 3 (protocol OK? = "no"). For groups 2 and 3, the closest distance was from the helicopter's path while circling. (When thinking about what was a "survey line," or "your circle," don't include the part of the flight path when flown closer to group 2 or 3 in order to go count those groups and record covariates for those groups. Use the closest distance that the observers came to the group, other than the flight path followed to get a closer look at that group). In this example, that distance was " $<1 / 4$ " for group 2, and " $1 / 2-1$ " for group 3. The helicopter's planned path along the transect lines are red, then green, with 1 mile transect spacing. 


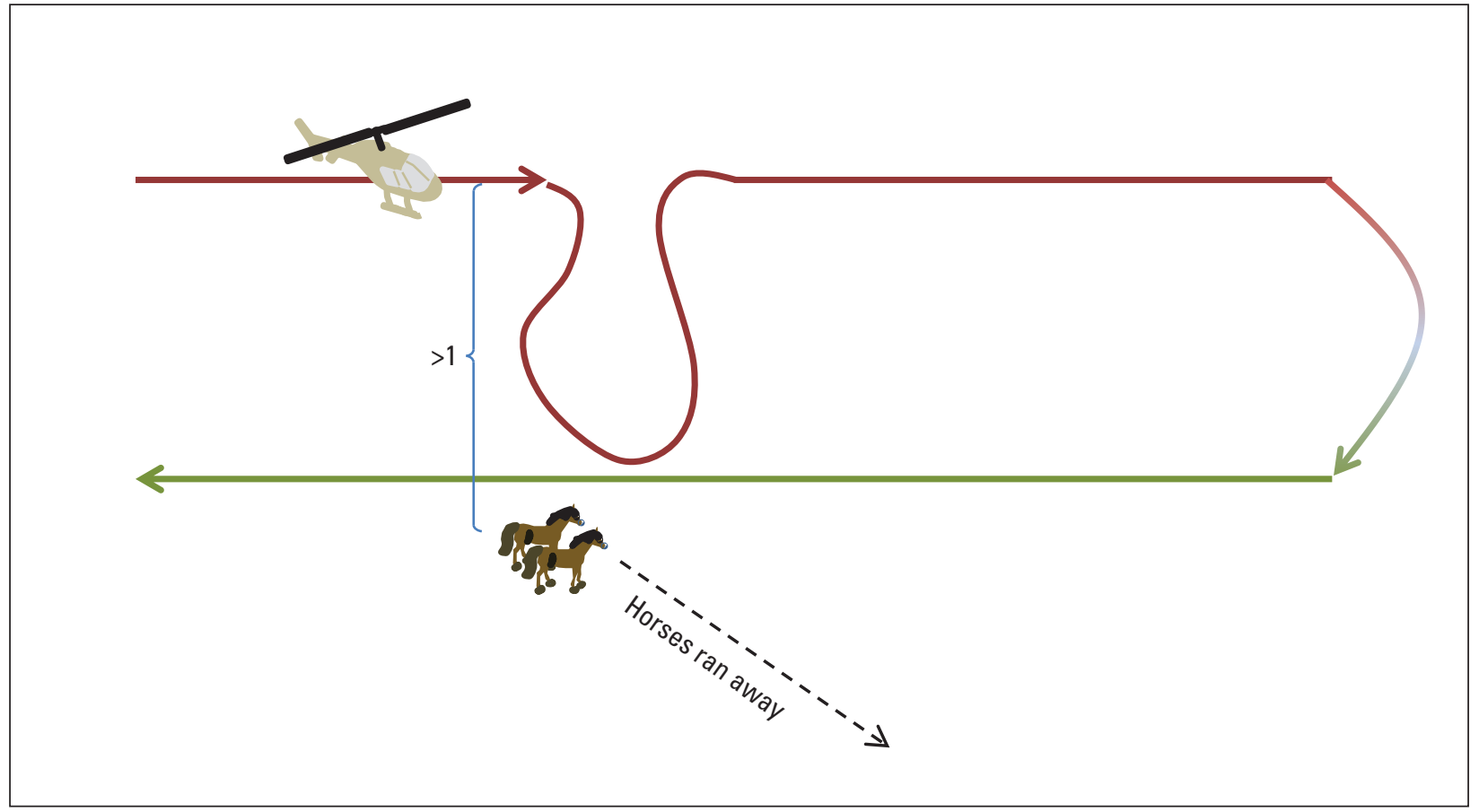

Figure 14. In the diagram of this situation, a horse group is detected by the front seat observers, more than one transect away, and running further away. The front seat observer decides that the horses must be counted right away before they are gone, even though the backseat observers did not yet have a chance to detect them. The flight is diverted from the red transect to go count the horses, and they are not seen again on the green transect (or ever again in the survey). Record the closest distance from where the horse group was when it was first detected to the survey line where the crew was when the group was detected. When thinking about what was a "survey line," don't include the part of the flight path when it was flown closer to go count that group. Even though the flight approached very close to the group to count them accurately, the horses were first detected $>1$ mile from the transect line the crew was on when the group was detected, so record that distance ( $>1$ mile). The protocol was not followed in this situation (Protocol OK = no) because the helicopter diverted before back seat observers had their chance to detect the group. The helicopter's planned path along the transect lines are red, then green, with 1 mile transect spacing. 


\section{After Surveys}

When the survey ends, and the aircraft starts to ferry to the refueling or landing site, the data recorder writes down the Survey Stop time on the Flight Information Form. Upon landing, the data recorder fills in the Landing arrival time on the Flight Information Form and the ending Hobbs meter number (Hobbs Stop).

Immediately after every survey flight (that is, at refueling stops and at the end of the day), the WHB specialist oversees checking all datasheets for legibility and completeness. Flag any missing data and try to fill in missing data based on the best information possible (immediate recollections, discussions with other observers, photographs).

If the surveys were over military installations where photographs may be censored, due to Department of Defense mission needs, try to review Total Count for large groups at the landing zone right away before turning over photographs to military censors.

The crew and pilot will then confer and debrief, discussing any flight safety issues and any survey-related issues that arose. Record any relevant notes that will go into the report about the survey, including what went well, and what could be done differently next time.

The WHB specialist may need to consult with a GIS specialist to download and process the GPS flight line and animal location data, prepare daily flight maps, and get mapping equipment ready for the next flight. Recharge any laptops and GPS units overnight.

Download and rename photographic images of groups back in the office (see SOP 4. "Processing Digital Photographs") as soon as possible, ideally within a day after the flight.

Within a week of flights, write notes that will contribute to the survey report. Include

- weather and flight conditions, including lessons learned;

- HMAs, HAs, WHTs surveyed in whole or part (with notes about any partial survey);

- unusual observations; and

- observations of forage and vegetation condition in the surveyed areas.

For surveys in multiple jurisdictions, WHB specialists should solicit and compile feedback from other project participants about what worked well, what did not work well, and what could be improved in future surveys.

Within 60 days of flights, enter the data into the most current Excel spreadsheet, verify the accuracy of the data entry, and send all files to the USGS aerial survey specialist for analysis. Sooner is better, in case there are inconsistencies on the datasheets and the WHB specialist will need to recall or discuss a question. After entering data, make a separate opportunity to review and verify that each line of data is entered correctly and completely. The bottom of each datasheet has a place to note when data were entered on the spreadsheet, and when they were verified.

\section{Field Form Handling Procedures}

All field data forms are part of the permanent record for project data, so they should be handled in a way that preserves their future interpretability and information content. If changes to data on the forms need to be made subsequent to data collection, the original values should not be erased or otherwise rendered illegible. Instead, changes should be made as follows:

- Draw a horizontal line through the original value and write the new value adjacent to the original value with the date and initials of the person making the change.

- All corrections should be accompanied by a written explanation in the appropriate notes section on the field form. These notes should also be dated and initialed.

- If possible, edits and revisions should be made in a different color ink to make it easier for subsequent viewers to be able to retrace the edit history.

- Edits should be made on the original field forms and on any photocopied forms.

These procedures should be followed throughout data entry and data revision. After all data have been entered and verified in the spreadsheet or database, the original (marked-up) data sheets should be scanned as PDF documents, sent to the USGS aerial survey specialist, and archived at the local BLM office. 


\section{Renaming Scanned Field Data Forms}

Rename the .pdf file of the scanned data forms with the following standard file name format:

- Forms_;

- "yyyy_" is the year; and

- "HMAcode" is the 2-letter, 4-digit code that signifies each HMA, WHT, or HA.

For example, the scanned forms from Murderer's Creek surveys in 2014 would be renamed Forms_2014_OR0019.pdf.

Save a copy of the scanned forms at the district level (for archiving by the WHB specialist, and by the GIS specialist, to save with associated flight line and waypoint files) and send a copy to the USGS aerial survey specialist or BLM WHB research coordinator.

\section{Renaming Photo and Global Positioning System Files}

File name standards will help photos be searchable and organized in the larger context of a database of inventory records. These notes are also in the SOP for GPS use and the SOP for handling photos.

Refer to SOP 2."GPS Use" and in SOP 4. "Processing Digital Photographs" for guidelines.

\section{Data Entry and Analysis}

\section{Bureau of Land Management Wild Horse and Burro Program System}

Reminder: after surveys are complete, the WHB specialist or range conservation officer should enter the fact of survey completion for each surveyed HA or HMA into the BLM Wild Horse and Burro Program System. BLM Instructional Memorandum Number 2014-133 provides instructions (BLM, 2014).

\section{Entering Double-Observer Data onto Spreadsheet}

At this time (December 2019), all field form data are entered onto an Excel spreadsheet. Ask the USGS aerial survey biologist or BLM research coordinator to provide the latest version of the spreadsheet template and data entry training.

After data have been entered, the specialist must proof the data a second, separate time, to verify that data were entered correctly.

\section{Sending Double-Observer Data to U.S. Geological Survey for Analysis}

Send a folder containing copies of all the following files to the district GIS specialist, and to the USGS aerial survey specialist or BLM research coordinator:

- Document with notes about the surveys,

- Excel spreadsheet of observation data (or, notice that data were entered to the database),

- Processed and renamed flightline and waypoint files (that is, as shapefiles, .gdb, or .gpx files),

- Processed and renamed photographic files that were used to correct group size (.jpg), and

- Scanned copies of all data forms (.pdf format).

USGS will carry out analyses using an R script (R Development Core Team, 2018), in keeping with analysis protocols detailed in published SOPs. Data from each survey should be analyzed within two months after the entered, verified data have been sent to USGS.

USGS is developing a database that will hold observation data from aerial surveys, so that those data can be searchable by BLM and others in the future. This database should have data entry screens that are accessible over the internet. Part of the database will also hold the raw numbers of animals counted, and the estimated numbers of animals in the population.

USGS will send a draft of the non-interpretive memo for each survey (or set of surveys) for a courtesy review by the WHB specialists in field offices or district forests where those surveys took place, along with the state leads (and USFS lead, if 
applicable) and any other staff that the specialists or state leads request. The BLM (and USFS, if applicable) staff review this draft memo for quality assurance and quality control. After reviewing comments and revising the memo, USGS finalizes the analysis and memo, and sends it to the same staff.

\section{References Cited}

Bureau of Land Management (BLM), 2010, Wild horse and burro population inventory and estimation: Bureau of Land Management, Instruction Memorandum 2010-057. [Also available at https://www.blm.gov/policy/im-2010-057.]

Bureau of Land Management (BLM), 2014, Required timelines and naming conventions for entering data into the wild horse and burro program system and recording/reporting requirements for other data: Bureau of Land Management, Instruction Memorandum 2014-133, accessed November 14, 2019, at https://www.blm.gov/policy/im-2014-133.

Cogan, R.D., and Diefenbach, D.R., 1998, Effect of undercounting and model selection on a sightability-adjustment estimator for elk: Journal of Wildlife Management, v. 62, no. 1, p. 269-279. [Also available at https://www.jstor.org/ stable/3802288.]

Lubow, B.C., and Ransom, J.I., 2009, Validating aerial photographic mark-recapture for naturally marked feral horses: Journal of Wildlife Management, v. 73, no. 8, p. 1420-1429. [Also available at https://www.jstor.org/stable/20616812.]

Lubow, B.C., and Ransom, J.I., 2016, Practical bias correction in aerial surveys of large mammals - Validation of hybrid double-observer with sightability method against known abundance of feral horse (Equus caballus) populations: PLoS-ONE, v. 11, no. 5, accessed December 3, 2019, at https://doi.org/10.1371/journal.pone.0154902.
Minnesota Department of Natural Resources, 2008, DNRGarmin (DNRGPS): St. Paul, Minnesota Department of Natural Resources. [Also available at https://www.dnr. state.mn.us/mis/gis/tools/arcview/extensions/DNRGarmin/ DNRGarmin.html.

National Wildfire Coordinating Group (NWCG), 2019, Interagency helicopter operations guide; PMS 510: National Interagency Aviation Committee, National Wildfire Coordinating Group, accessed November 14, 2019, at https://www. nwcg.gov/publications/510.

R Development Core Team, 2018, R-A language and environment for statistical computing: Vienna, Austria, The R Foundation, accessed November 14, 2019, at https:// www.r-project.org.

Ransom, J.I., 2012, Detection probability in aerial surveys of feral horses: Journal of Wildlife Management, v. 76, issue 2, p. 299-307. [Also available at https://doi.org/10.1002/ jwmg.204.]

Schoenecker, K., Lubow, B., Ziegenfuss, L., and Mao, J., 2006, 2005 annual progress report-Elk and bison gazing ecology in the Great Sand Dunes complex of lands: U.S Geological Survey Open-File Report 2006-1267. [Also available at https://doi.org/10.3133/ofr20061267.].

Unsworth, J.W., Leban, F.A., Leptich, D.J., Garton, E.O., and Zager, P., 1994, Aerial survey-User's manual with practical tips for designing and conducting aerial big game surveys: Boise, Idaho Department of Fish and Game, 84 p. [Available for viewing from the University of Idaho Library, OCLC number 41849892.] 


\section{Standard Operating Procedure 2-Global Positioning System Use}

\section{Overview}

This SOP provides background and guidelines for GPS use. More detailed instructions for operating and building background maps will depend on specific GPS models and software.

\section{Introduction}

GPS receivers are used to navigate, follow planned survey flight lines, and mark waypoints to map animal observation locations. GPS receivers help flight crews increase survey efficiency, determine whether or not survey areas are surveyed adequately, and estimate flight times. Documenting flight times ferrying to and from surveyed areas can help determine future planning and costs for aircraft use.

The combination of hardcopy or image file maps distributed to dispatch and the helibase and GPS receivers in aircraft is critical for crew safety. This is particularly important if two helicopters are conducting horse surveys at the same time.

Having pre-designated flight lines allows the pilot to focus on safe flight and on navigating the survey route and allows observers to focus on searching for animals. It should be explained clearly to the pilot before flight that the pilot is expected to follow the flight lines (allowing for safety as the first priority), maintaining a safe VFR cruising altitude to and from the survey area, then descending for conduct of the survey. The crew should refer to the record of actual flight lines and compare them to planned flight lines when convenient (that is, during refueling breaks between flights).

Observers should not generally help the pilot with navigating unless the pilot is having difficulty. The crew should have paper maps in addition to a GPS-enabled electronic map device. Paper maps will serve as navigational safety back-up in the event of electronic equipment failure or lack of GPS satellite availability.

Specific methods and equipment configurations will change as equipment and software changes. The "Directions for GPS Use" section that follow are general steps. More detailed instructions for operating and building background maps for specific GPS models and software should come from GIS staff and (or) the USGS aerial survey specialist.

The necessary GPS and mapping equipment that should be on each aircraft are listed in the "Checklist for Survey Equipment" in appendix 4. 


\section{Directions for Global Positioning System Use}

I. Specific equipment needed:

a. One handheld GPS unit, plus one backup; and

b. Spare batteries or a way to connect handheld GPS receiver to external power source.

II. Handheld GPS set-up:

a. Set the receiver to store a coordinate location every 2 seconds, and

b. If the GPS receiver needs an external antenna, connect it to the GPS receiver to maximize satellite signal reception, and make sure the antenna has a clear view of the sky (the helicopter dashboard is a good place).

III. Flight line mapping:

a. Turn on GPS a few minutes prior to take-off to begin flight tracking.

IV. Observation mapping:
a. Mark a waypoint when the helicopter is over the horse group,
b. Make sure the data recorder writes down the horse group's waypoint number, and
c. Non-horse observations may be marked on the datasheet with Species clearly noted.

V. Daily post-survey GPS data download:

a. Download GPS data onto a laptop computer after each day's flights.

b. Rename GPS files with standard names. GPS files are, for example, .csv files with flight lines or waypoints, having time and Universal Transverse Mercator (UTM) coordinates:

i. $\quad$ GPS of actual flight line = GPS_yyyy_HerdCode_flt_xx.csv.

ii. GPS of waypoints = GPS_yyyy_HerdCode_wpt_xx.csv.

iii. For file naming where:

1. "yyyy" is the year;

2. "HerdCode is the 2-letter, 4-digit code that signifies each HMA (see app, 1, table 1.1); and

3. " $x x "$ is the flight number.

c. Back-up GPS files on a flash drive or a computer network.

d. Clear or delete files from the GPS receiver to make space for next day's flight.

VI. Recharge or replace GPS and laptop batteries. 


\section{Standard Operating Procedure 3-File Folder Structure}

Use a consistent file folder structure for records, such as the one below. The overarching folder is for WHB surveys (fig. 15). Files for aerial surveys are then arranged by fiscal year (from October 1 to September 30). Folders for each fiscal year include: Analysis (containing statistical analysis files, if available); Data (containing pdf scanned copies of paper datasheets, and spreadsheets of entered, proofed data); Documents (containing records on aviation safety, planning, results of analyses, and special considerations for military or wilderness planning, if needed); Images (containing photographs sorted according to whether or not they are "data photos" of use for analysis); and spatial information (records for flight path planning, actual flight paths flown, waypoints of observations, and associated GIS information).

Within the folder for Images, files within main folders are organized by year and Herd Code. For an example using Murderers Creek HMA, in Oregon, see section SOP 4, "Processing Digital Photographs."

Within the folder for Spatial Info, GIS_data includes shapefiles or geodatabase files used for planning or reflecting actual results. The GPS_data folder holds raw files from GPS units. The gpx folder and kml folder hold Garmin GPS files and Google Earth files, respectively. The Map_documents folder holds ArcMap projects, and the Maps_pdf folder holds exported maps in pdf format, such as flight plan maps for distribution to crew and dispatch.

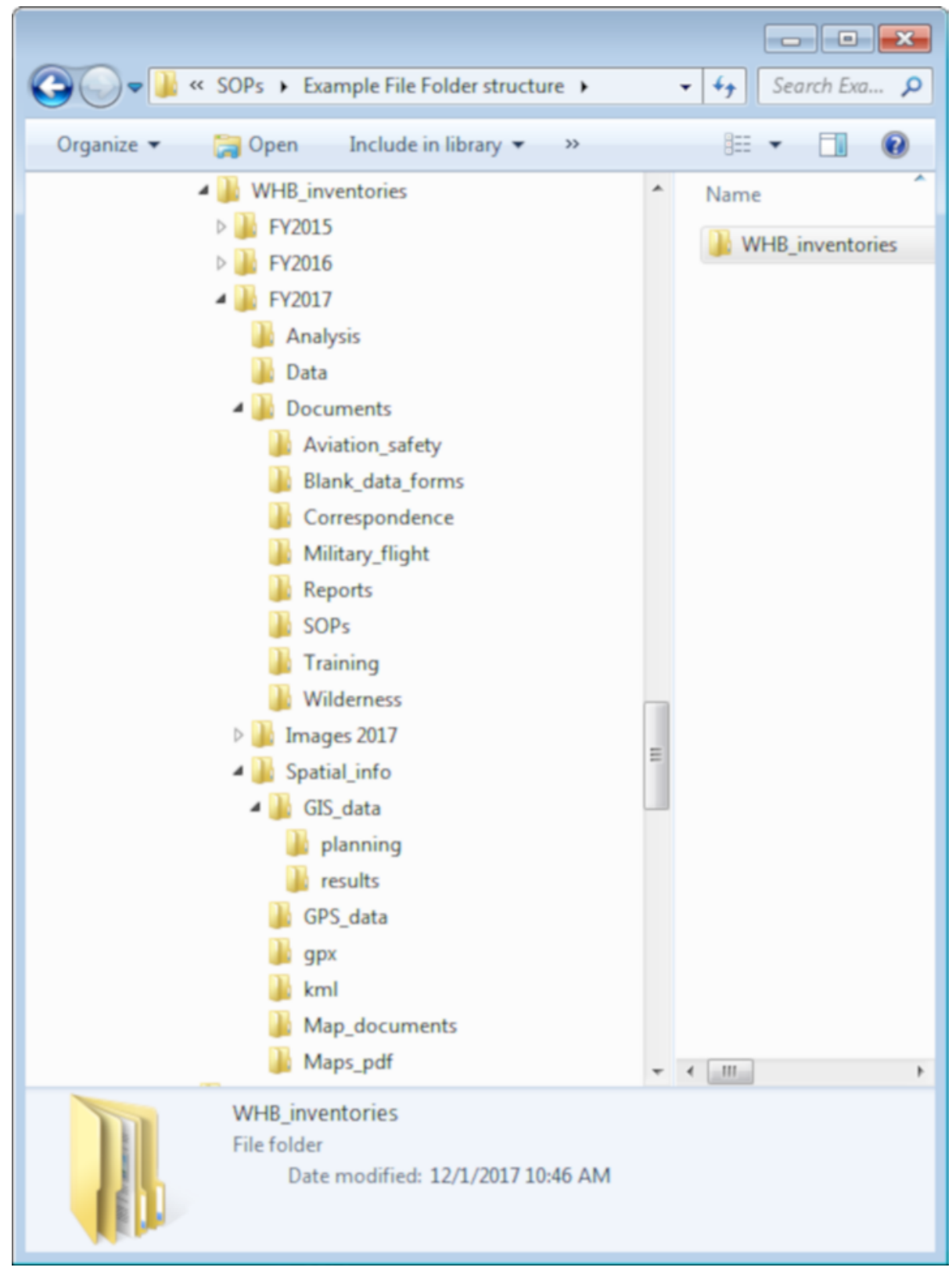

Figure 15. Screen snap of file folder structure for aerial survey documents for wild horse and burro (WHB) surveys. The overarching folder is for WHB surveys. Files for aerial surveys are then arranged by fiscal year. Folders for each fiscal year include: Analysis (containing statistical analysis files, if available); Data (containing pdf scanned copies of paper datasheets, and spreadsheets of entered, proofed data); Documents (containing records on aviation safety, planning, results of analyses, and special considerations for military or wilderness planning, if needed); Images (containing photographs sorted according to whether or not they are "data photos" of use for analysis); and spatial information (records for flight path planning, actual flight paths flown, waypoints of observations, and associated geographic information [GIS] information). 


\section{Standard Operating Procedure 4—Processing Digital Photographs}

\section{Overview}

This SOP describes procedures that will be used to process, name, and store photographic images that are collected that document size and composition of horse groups, or for any other project-related activities. Images that are acquired by other means (for example, downloaded from a website) are not project records and should be stored separately and named in such a way that they will not be mistaken for project records.

Photographs of horse groups should be processed following this SOP before data are entered into the data entry spreadsheet. Data forms from surveys (Flight Information Forms and Survey Datasheets) should be on hand during image processing because the forms may need to be updated based on information from the photographs.

If a photograph of an observed group reveals more animals than were recorded on the paper data form, then the group size recorded on the paper form should be corrected, using the number from the photograph. Cross out the previously recorded values for adults and foals, and enter the larger numbers based on the photo. The larger numbers must also be entered into the spreadsheet for analysis. If photographs for a particular group show a smaller number than was recorded on paper, do not change the number on the paper form; the assumption is that some of the animals in the group may not have been photographed. It is more common that observers undercount, rather than overcount groups of horses.

To effectively manage potentially hundreds of images requires a consistent method for downloading, naming, editing, and documenting. The general process for managing project images is as follows:

1. Prepare image workspace. Set up the folder directory structure before surveys.

2. Take photographs during the survey.

3. Download and process images;
a. Download the photograph files from the camera;
b. Copy and store the original, unedited versions;
c. Determine which horse groups or other landscape points of interest are represented by photographs;
d. Rename the files according to the prescribed convention detailed below;
e. Review horse group photographs for group size and composition;
f. Edit the photographs as needed, and delete unneeded or poor-quality photographs; and
g. Move the files into appropriate folders for storage.

4. Deliver image files for final storage.

\section{Data Photographs Defined}

Distinguish data photos from incidental or opportunistic photos. Data photos are those taken for one of three reasons:

1. To document group size and (or) composition of an observed horse group;

2. To document the hydrological, forage, or other conditions at specified locations;

3. To document survey conditions (for example, cloud cover).

Data photographs should be processed so that they can be linked to specific records in a database of horse group observations and survey flights that may be developed in the future. Data photographs are stored in a manner that permits the creation of database links in the future. Other photos, for example, of survey crew members at work, or photographs showing habitat conditions in general, are not data photographs. They may be retained but will not necessarily be linked with flight records. 


\section{Image Acquisition}

For details on image acquisition, see section "SOP 1. Photography." When survey crews find groups of $\sim 20$ or more horses, those groups must be photographed, preferably so that all the horses in the group are visible in one photograph. Encourage the pilot to stay back, away from the group, to take pictures first before flying closer. It is highly recommended to take photos of any group of $\sim 10$ or more animals. Capture images at a high enough resolution to classify horses into age categories, but keep in mind computer memory space limitations. It may be necessary to store images on an external device, such as a hard drive. A recommended minimum raw resolution is $1,600 \times 1,200$ pixels (approximately 2 megapixels). Higher resolutions may be available but are currently undesirable from the perspective of data storage and information content.

\section{Image File Folder Structure}

The district WHB specialist (or a designee) should create a main Images folder in the district computer workspace for filing photographs. As of the writing of these SOPs, the photographs should be stored at the district office, and should be shared with USGS or the BLM WHB research coordinator after they have been processed and properly filed.

Before each season's data collection, within the larger Images folder, the workspace subfolders are as follows:

\begin{tabular}{|c|c|c|}
\hline Year_HMA, HA, or WHT & & $\begin{array}{l}\text { The appropriate year, followed by 2-letter + 4-digit code name of HMA, HA, or WHT. } \\
\text { Typing out the full HMA is optional. }\end{array}$ \\
\hline 1_Originals & [Download date] & $\begin{array}{l}\text { Renamed, but otherwise unedited image file copies } \\
\text { Arranged by download date to preserve file names }\end{array}$ \\
\hline 2_Processing & [Flight number] & $\begin{array}{l}\text { Processing workspace } \\
\text { Arranged in folders by flight number }\end{array}$ \\
\hline 3_Data_Groups & [Flight number] & $\begin{array}{l}\text { Required data images taken of observed WHB groups } \\
\text { Arranged in folders by flight number }\end{array}$ \\
\hline 4_Data_Phenology & [Flight number] & $\begin{array}{l}\text { Required data images taken at hydrology, forage, or phenology photo points } \\
\text { Arranged in folders by flight number }\end{array}$ \\
\hline 5_Miscellaneous & [Flight number] & $\begin{array}{l}\text { Non-data images taken incidental to the survey } \\
\text { Arranged in folders by flight number }\end{array}$ \\
\hline 6_Flight_Conditions & [Flight number] & $\begin{array}{l}\text { Non-data images taken at start and end of flight (optional) } \\
\text { Arranged in folders by flight number }\end{array}$ \\
\hline 7_Other_Sources & & Images acquired from other sources (that is, non-record) \\
\hline
\end{tabular}

This folder structure (fig. 16) permits data images to be stored and managed separately from non-record and miscellaneous images collected during the course of the project. This structure also provides separate space for image processing and storage of originals.

In all cases, folder names should follow these guidelines:

- No spaces or special characters in the folder name,

- Use the underbar ("_") character to separate words in folder names,

- Try to limit folder names to 30 characters or fewer,

- Date names should be formatted as YYYYMMDD, and

- Use full year (that is, 2013). 


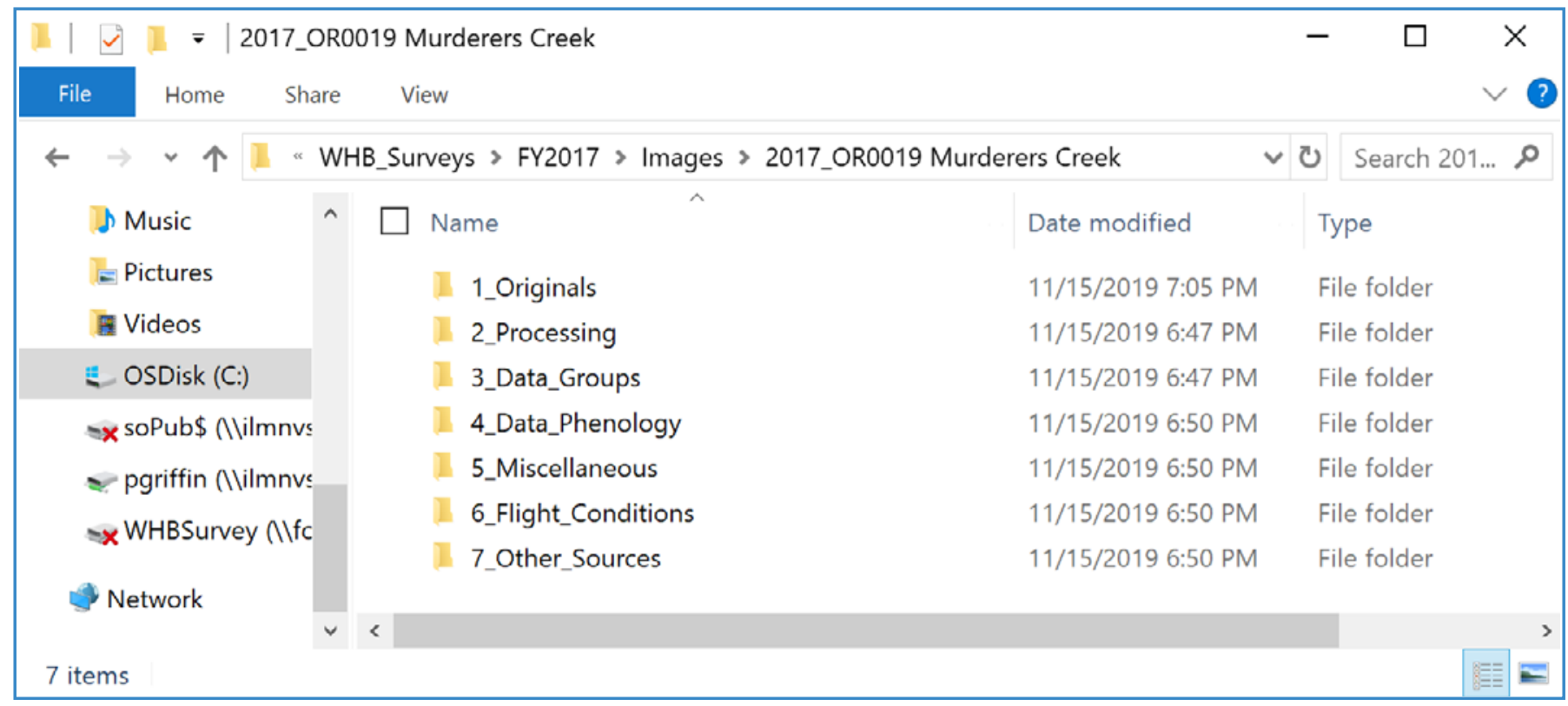

Figure 16. Screen snap of an example file folder structure for wild horse and burro aerial survey image files. These images are saved in the 2017 Images folder for Murderers Creek Herd Management Area (HMA; herd code OR0019. For herd codes see app. 1, table 1.1.)

\section{Image File Naming Standards}

File name standards will help make photographs searchable and organized in the larger context of a future database of inventory records. In all cases, saved image names should follow these guidelines:

- No spaces or special characters in the file name;

- Use the underbar (" " ") character to separate file name components;

- Try to limit file names to 30 characters or fewer, up to a maximum of 50 characters;

- 2-letter + 4-digit herd code, year, flight number, and horse/burro group waypoint number or photo point number should be included in the file name; and

- Dates should be formatted as YYYYMMDD (this leads to better sorting).

The image file name should consist of the following parts:

- 2-letter + 4-digit Herd code for HMA, HA, or WHT;

- Year (2013,_2014,and so forth);

- Flight number (_f\#\#);

- Species (_h for horses; _b for burros);

- Horse/burro group waypoint number $(\mathrm{w} \# \# \#)$ or phenology photograph point number $\left(\_\# \#\right)$;

- Optional: a sequential letter if multiple images were captured (a, b, c, and so forth); and

- Optional: abbreviated original camera-given file name of the image (that is, IMG_0756).

Examples of proper file names:

-WY0035_2014_f4_h_w23b.jpg Lost Creek HMA surveys of 2014, flight 4, horse group, waypoint 23, the second of at least 2 saved photos

•AZ0011_2015_f10_b_w165.jpg Cibola-Trigo HMA surveys of 2015, flight 10, burro group, waypoint 165

• NV0619_2016_f3_p2.jpg Reveille HMA surveys of 2016, flight 3, photo of forage point number 2

- OR0002_2014_f1_h_w17a.jpg Beaty's Butte HMA surveys of 2014, flight 1, horse group, waypoint17, first of the saved photos 


\section{Downloading and Processing Procedures}

A. Under the appropriate 1_Originals subfolder, create a subfolder for the download date (for example, 20120715). Other suffixes may be used to distinguish downloads when more than one camera is downloaded on the same date (for example, "a," "b," and so forth).

B. Download the raw, unedited images from the camera into the 1_Originals folder, within the subfolder with the appropriate date.

C. Copy the images to the 2_Processing folder. After copying, set the photographs that are saved in the 1_Originals folder as read-only by right clicking in Windows Explorer and checking the appropriate box. These originals serve as backups in case of unintended file alterations (for example, incorrect names applied, file deletion, loss of resolution, or loss of image metadata upon rotation).

D. Determine which photographs correspond to horse groups of $\sim 10$ or more, and which are hydrology, forage, or phenology photograph points.

a. Open the 2_Processing folder that contains the photos and select View $=>$ Details from the uppermost menu (fig. 17); this creates a detailed list of the files. To see the time that each photograph was created, right-click on the gray bar just above the file names; this shows a pick list of file attributes that can be displayed.

b. At the bottom of that list, select "More" (fig. 18).

c. From the Details list, check "Date taken" (fig. 19). Note: the "Date created" choice here only reflects the date when the file was copied to the folder - not the date or time when the photo was taken. Based on the "Date taken" field, determine which photographs match a time for a large horse group observation, or for a phenology photo point.

d. Find what time the photographs were taken and compare this to the times recorded for each horse group (on the Survey Datasheet).

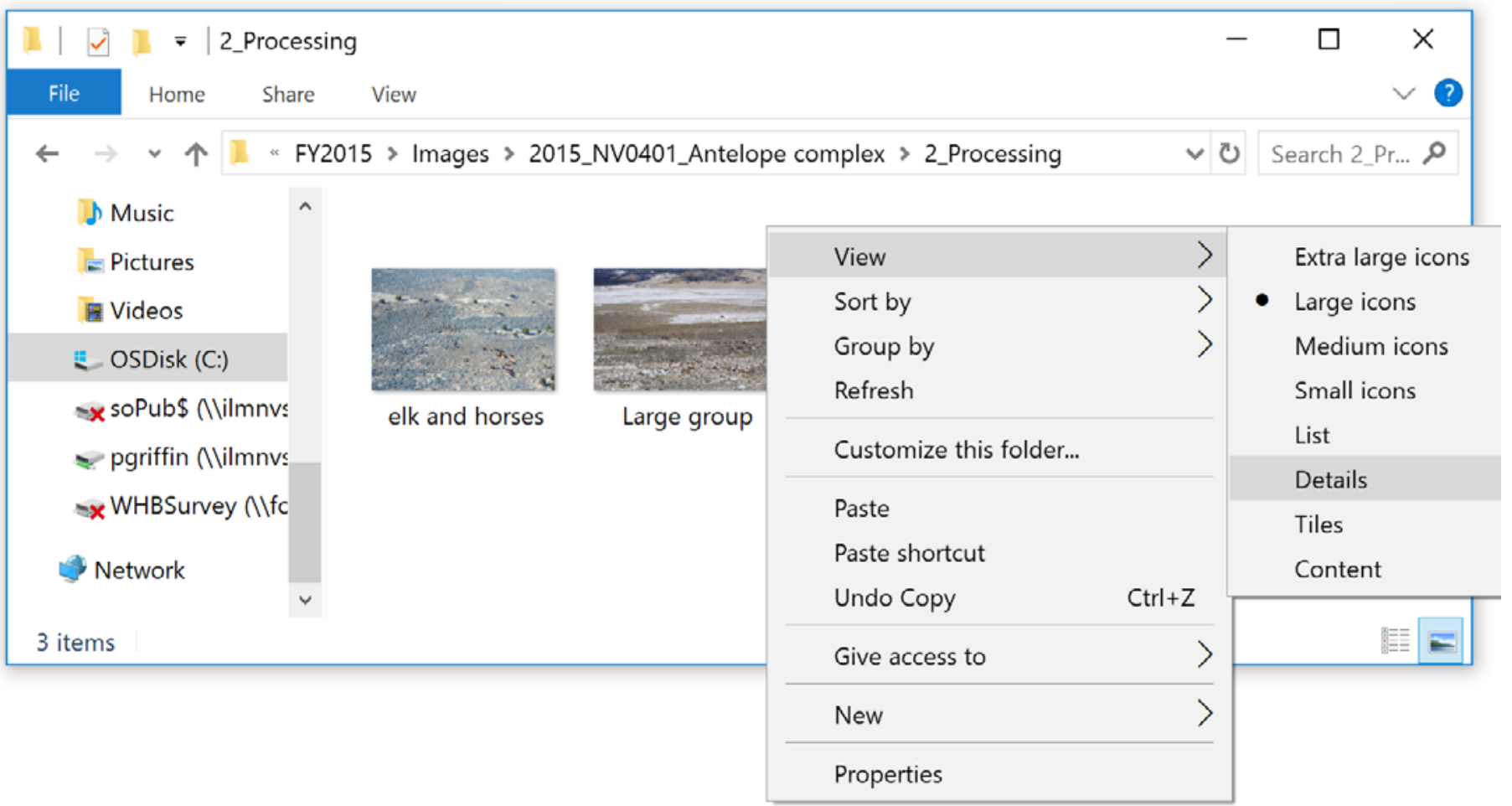

Figure 17. Screen snap of an example file folder structure for wild horse and burro aerial survey image files for downloading and processing procedures. Select View $\rightarrow$ Details to see a detailed list of the photos in the 2_Processing folder. 


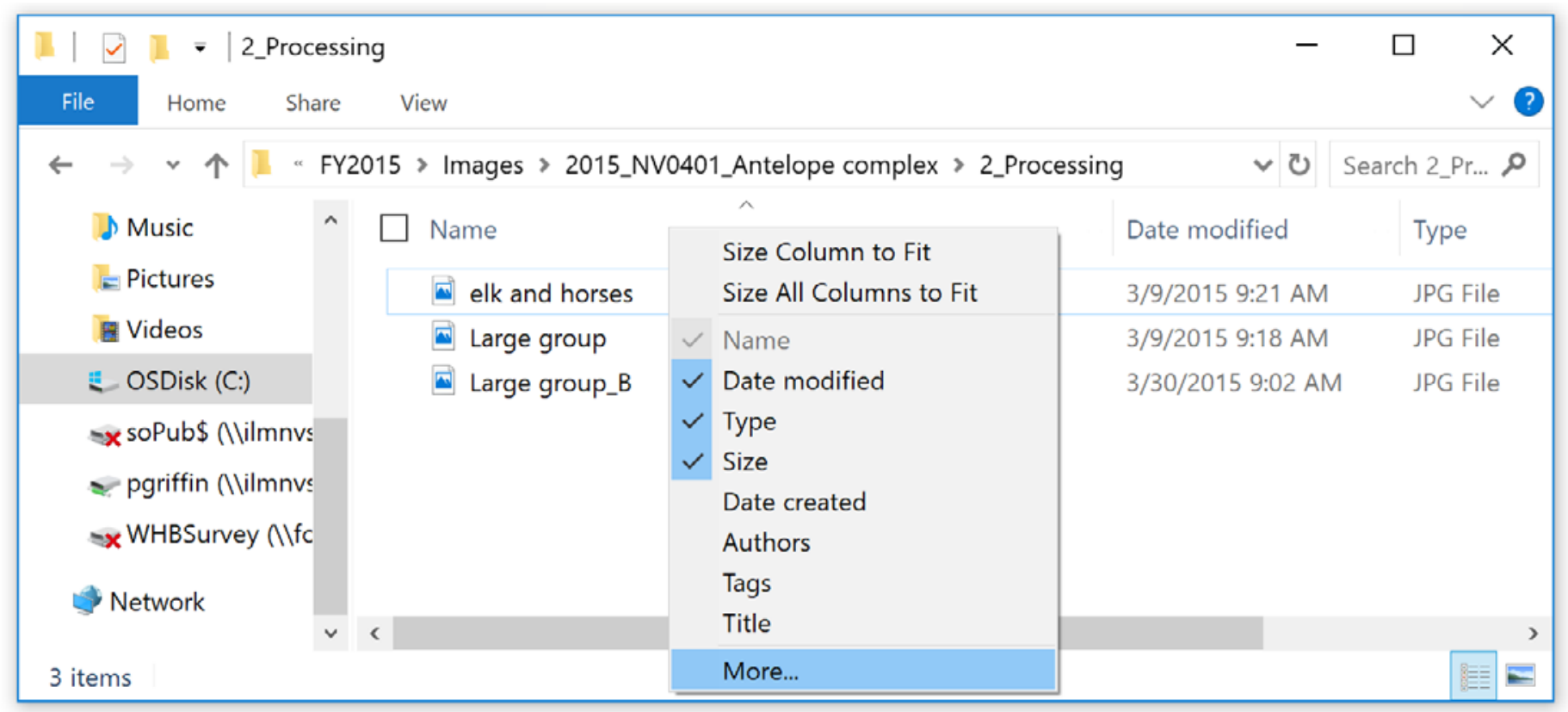

Figure 18. Screen snap of an example file folder structure for wild horse and burro aerial survey image files for downloading and processing procedures. Right click on the "Name" bar above the photo files and select "More..." to see more detail options about the photo files.

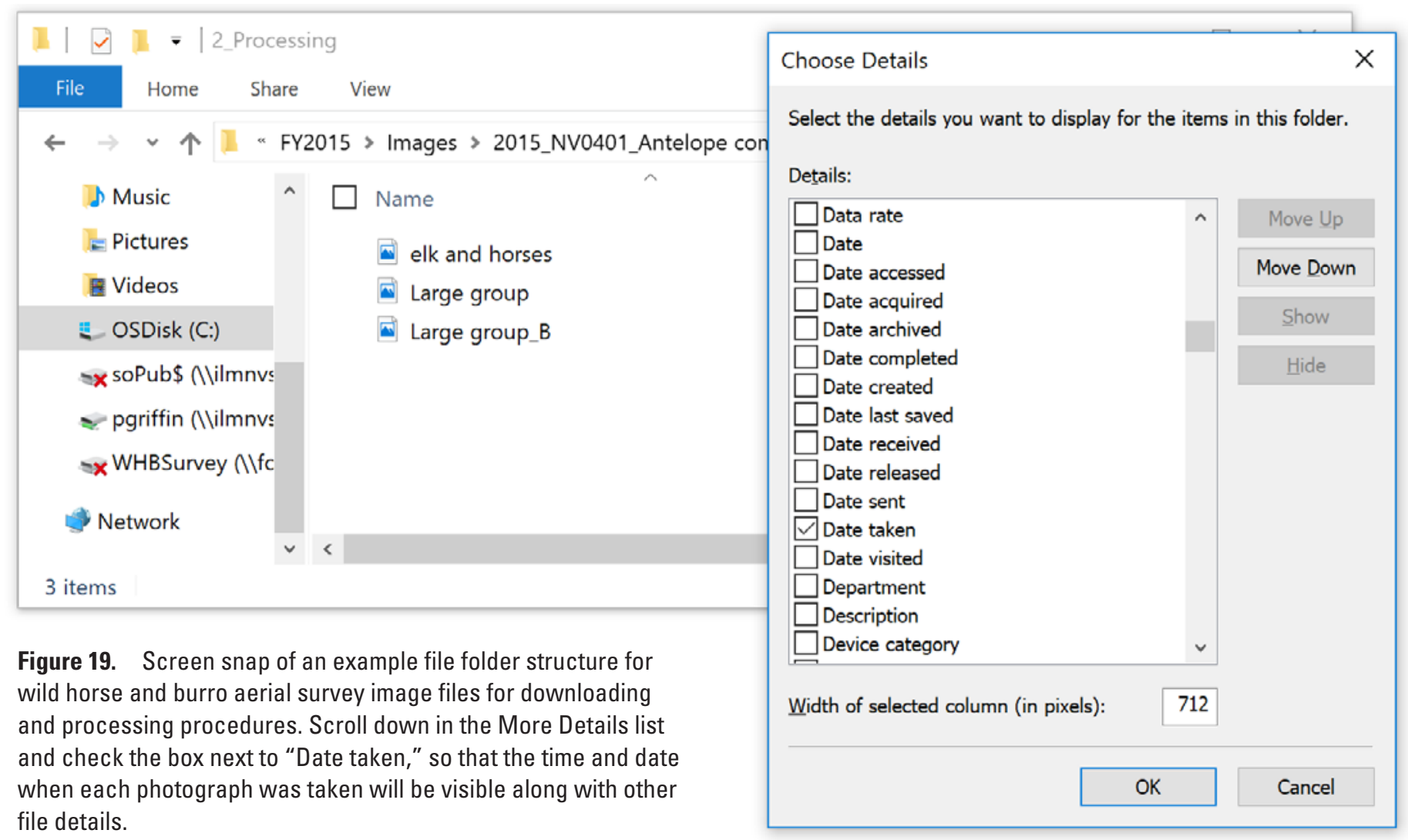


E. Rename the images according to the "SOP 4. Image File Naming Standards" section, above.

a. If changing the numbers of adults and foals on the Survey Datasheet, any annotations should be done in a different color ink from the original color used in the aircraft, after first drawing a line through the original entry (for more information, see "SOP 1. Field Form Handling Procedures."

b. In some cases, renaming may be most efficiently done as a batch using image processing software such as Microsoft Office Picture Manager, which allows a standard prefix or suffix to be added to the camera file name. After batch renaming, a descriptive component may be added manually to each file name.

F. Review the photographs for group size and composition before any data are entered into an Excel spreadsheet or (once it is developed) the database.

a. To evaluate group size, use the photograph of a given horse group that includes the greatest number of animals.

b. Count the number of adults and foals. This process may be somewhat subjective. The total number of horses in the group should generally be the greater of: the group size originally written on the Survey Datasheet; or the total number visible in the best, most complete photograph. If there are color markings on the horses, and there is a larger total, based on two or more photographs of the same group (if this happens), be sure that the time stamp on the photographs shows that it is the same group.

c. If the total number of horses in the photo is less than that written on the Survey Datasheet, do not change the value for group size on the data form (this reflects an assumption that the number of horses counted in the air may have included horses that were not in the photograph). On the Comments line of the Survey Datasheet, note the number of animals that are not visible in the picture (that is, "Photo not of complete group; 2 adults out of picture.").

d. If the number of horses in the photograph is larger than that on the Survey Datasheet, then increase the value on the data form to match the evidence from the photograph. The decision about whether or not to update the recorded composition values may depend on whether the photographer included all the animals in the group in the photograph. Counts can sometimes be based on several photographs.

e. Print a hard copy of any photograph that is used to update the group size or composition of any group, label the hard copy with the HMA name, date, flight number and waypoint number, then attach it to the original Survey Datasheets, for archiving.

f. Edit or delete photographs, as necessary.

i. Delete poor quality, repeat, or other unnecessary photographs. Low quality photographs may be kept if the subject is unique or irreplaceable.

ii. Rotate the image to make the horizon level.

iii. Crop photographs to remove edge areas that distract from the subject.

G. Move the image files that are to be retained and possibly linked in the database to the appropriate folder.

a. Properly named photos of horse or burro groups should go into the 3_Data_Groups folder.

b. Photographs of hydrology, forage condition, or phenology points should go into the 4_Data_Phenlogy folder.

c. Photographs of crew or other incidental subjects should go into the 5_Miscellaneous folder.

d. Photographs of survey conditions should go in the 6_Flight_Conditions folder.

e. Photographs taken by non-agency staff should go in the 7_Other_Sources folder.

H. Archive copies of the photographs at the district computer system, using the image file folder structure shown above.

I. Send copies of data photographs, renamed and saved within appropriate subfolders, to USGS aerial survey specialist or BLM WHB research coordinator. 
J. Once the appropriate person at the BLM National Operations Center (https://www.blm.gov/services/national-operationscenter) has been identified, send copies of the data photographs, renamed and saved within appropriate subfolders, to that person (not yet determined as of January 2020).

K. Photographs of potential interest to a wider audience should be copied to a different folder where they can be more broadly accessible.

a. Notably good photographs taken by BLM staff should be shared by email with the BLM visual information specialist and BLM WHB WO262 marketing specialist.

b. Notably good photographs taken by USFS staff should be shared with the local district's public affairs officer, and with the WHB inter-regional program coordinator.

L. Delete files from the 2_Processing folder between downloads to minimize the chance for accidental deletion or overwriting of needed files.

\section{Post-Survey Cleanup Procedures and Quality Control}

After each set of surveys, the wild horse and burro specialist (or a designee) should:

1. Review the workspace folders to make sure that all images are properly named, filed, and accounted for.

2. Set the images in the project workspace to read-only to prevent unintended changes.

3. The 2_Processing folder should be emptied and may then be deleted.

4. Files in the 7_Other_Sources folder may be re-filed as appropriate.

5. The contents of the 1_Originals folder may be deleted once all desired files are accounted for. Originals may be retained as desired, depending on storage limitations.

6. Copy the entire contents of the Images subfolder from the seasonal workspace to the main project workspace and delete the images subfolders from the seasonal workspace.

7. If it is necessary to conserve network server memory space, save photographs from the 1_Originals folders offline. 


\section{Standard Operating Procedure 5—Reference for Analyzing Double-Observer Data}

\section{Overview}

This SOP refers to methods for analyzing double-observer data. Ekernas and Lubow (2019) present the general structure of data files that are associated with the results, including scanned copies of survey data forms, spreadsheets of survey data, spatial (GIS) files with flight lines and waypoints at recorded locations, data photos, the R-script for data analysis, and a copy of the report with abundance estimates.

\section{Introduction}

In the context of this SOP, we define an "estimate" of herd size to be an expected value of the true herd size based on the survey data. As defined here, an estimate is not a guess. Estimates have associated confidence intervals around them to acknowledge that there is uncertainty in the estimate, but to also bracket that uncertainty quantitatively.

Analytical methods that use data from double-observer surveys to obtain estimates of herd size are presented in Ekernas and Lubow (2019).

\section{Reference Cited}

Ekernas, L.S., and Lubow, B.C., 2019, R script to analyze simultaneous double observer wild horse and burro aerial surveys: U.S. Geological Survey software release, URL: https://code.chs.usgs.gov/fort/whas, doi: https:// doi.org/10.5066/P946MHTZ. 


\section{Standard Operating Procedure 6—Preparing Flight Lines for Aerial Surveys}

\section{Overview}

This SOP describes the general approach to take when preparing or revising flight lines that will be followed during aerial surveys. The SOP lists steps that should be taken during this process, but the reader should understand that there is no perfect recipe for creating the layout of flight lines. The goal of flight line planning is to allow aerial observers the opportunity to have complete visual coverage of the area searched.

\section{Steps}

1. Define the area to be surveyed; this should encompass the entire population of horses or burros that use the targeted HMAs, HAs, WHTs, or complex.

2. Prepare a project in esri's ArcGIS or other similar software. Use a North American Datum 1983 (NAD 83) UTM projection so that distances between locations can be easily judged.

a. The project should include spatial data layers (that is, shapefiles or feature classes) showing: the boundaries of HMAs, HAs, WHTs; roads, fences, designated landing zones, aerial hazards (that is, from online FAA sectional aeronautical charts), topography, and satellite images of vegetation.

b. Prepare or add a spatial data layer showing a grid of $1 / 4$ mile lines; this can be useful in laying flight lines out that are spaced at consistent distances. Create a new polyline shapefile or feature class to hold the planned flight lines. Edit the feature class so that new line features can be added in GIS.

\section{Spacing}

Discuss the appropriate line spacing with the USGS aerial survey specialist and with local specialists who have flown the survey area before, or who have previously flown in comparable areas.

Typical line spacing distances are suggested in the table below. Local decisions to vary from these starting points are absolutely fine. Keeping in mind that the goal is to have complete visual coverage of the surveyed area, the pattern in the table below is that tighter flight lines are required in areas where poorer visibility would be expected. Burros are harder to see, so flight line spacing is closer for burro surveys than horse surveys. 
Table 1. Preparing flight lines for wild horse and burro double-observer aerial surveys. Below are typical transect spacing in different conditions.

[Fixed wing is not the preferred choice for mountainous topography, but there are some cases where the majority of the area to be surveyed is best done with a fixed wing platform, and where a small fraction of the total can be covered with fixed wing, where the flight lines are laid out to follow the topographic relief.]

\begin{tabular}{|c|c|c|c|}
\hline Aircraft & Topography & Vegetation & Spacing (miles) \\
\hline \multicolumn{4}{|c|}{ Horse (Equus caballus) } \\
\hline Fixed wing & Flat & Open & 2 \\
\hline Fixed wing & Flat & Pinyon-juniper & 0.75 \\
\hline Fixed wing & Gullies & Open & 1.5 \\
\hline Helicopter & Flat & Shrubby & $1.25-1.5$ \\
\hline Helicopter & Flat & Pinyon-juniper & $0.5-0.75$ \\
\hline Helicopter & Flat & Thick forest & 0.25 \\
\hline Helicopter & Gullies & Open & $1-1.25$ \\
\hline Helicopter & Mountainous & Open & $0.5-0.75$ \\
\hline Helicopter & Mountainous & Shrubby & 0.5 \\
\hline Helicopter & Mountainous & Pinyon-juniper & $0.25-0.5$ \\
\hline Helicopter & Mountainous & Thick forest & 0.25 \\
\hline \multicolumn{4}{|c|}{ Burro (Equus asinus) } \\
\hline Helicopter & Flat & Open & $0.5-0.75$ \\
\hline Helicopter & Flat & Shrubby & $0.4-0.7$ \\
\hline Helicopter & Flat & Pinyon-juniper & $0.25-0.5$ \\
\hline Helicopter & Flat & Thick forest & $0.15-0.25$ \\
\hline Helicopter & Mountainous & Thick forest & $0.15-0.25$ \\
\hline
\end{tabular}




\section{Standard Operating Procedures for Wild Horse and Burro Double-Observer Aerial Surveys}

\section{Planning Survey Segments}

- Identify the start point for the survey. This need not be mapped, but should be known and discussed in pre-flight mission briefings.

- Be aware of the approximate flight time that the loaded aircraft will allow, taking into consideration expected load calculations. Multiply the flight time by the approximate survey speed to determine the approximate distance that the aircraft can fly between refueling (that is, a survey segment). Plan survey segments so that lengths correspond to a shorter distance than fuel range. Plan the locations of refueling to minimize ferry time that is not spent surveying.

- Plan the placement of survey segments so that it will be possible to survey areas that are bounded by topographic breaks, fences, and other barriers in their entirety within a single flight. The goal is to survey areas where horses can move freely all in a single flight.

- Plan transect placement to avoid any known aerial hazards (based on the most recent FAA sectional aeronautical charts and other available information).

\section{Map and Print Planned Survey Segments}

- Prepare maps showing the planned survey segments, planned landing zones, and other local landmarks, overlaid on satellite imagery of the planned survey area (fig. 20). Include the HMA boundaries.

- Use the most recent FAA sectional aeronautical charts to identify aerial hazards, and map those.

- Indicate where each survey segment starts, so the direction of travel is clear.

- Include a scale bar. Include the mileage for each survey segment (and ferry distance).

- Circulate flight plan maps to dispatch, aviation managers, and other crew members. 


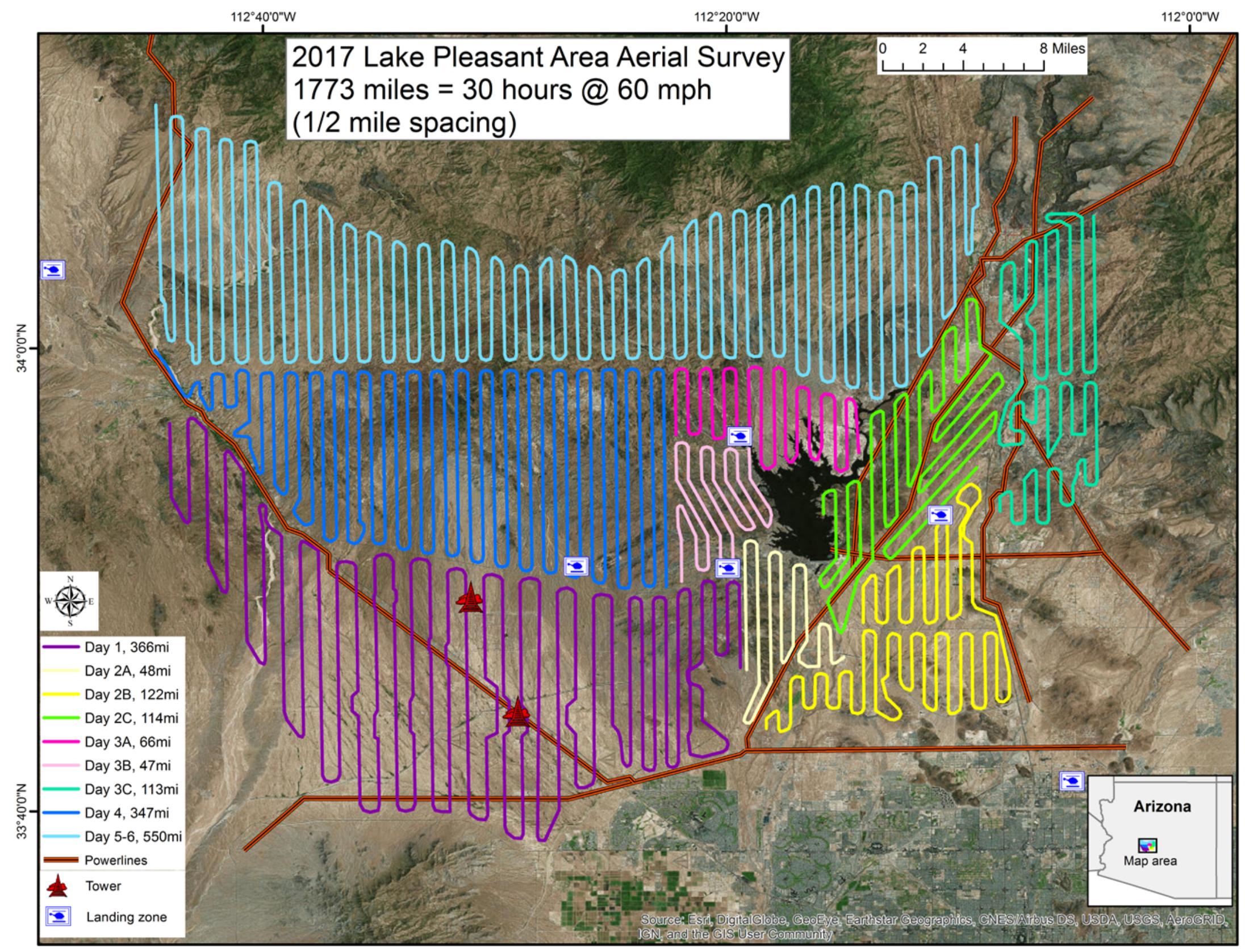

Figure 20. Image with diagram of flight lines for aerial surveys for wild burros. This is an example flight plan map for the Lake Pleasant, Arizona, survey area.

The legend includes hazards and mileage for each flight segment, a scale, north arrow, and an inset location map. 


\section{Standard Operating Procedure 7-Principles for Projecting Population Size}

\section{Overview}

This SOP describes general principles that can be followed to project future abundance for horses and burros in a given HMA or HA managed by the BLM, based on estimated abundance values from aerial surveys and population growth rates. Annual projections made following these guidelines should reflect the approximate numbers of horses and burros present for the herd that uses each HMA, standardized to represent the expected abundance on March 1 of each year. However, guidelines in this SOP do not represent BLM policy, and BLM is not required to follow these guidelines when estimating and reporting herd abundance each year.

\section{Introduction}

The BLM WHB Program reports the approximate local abundance of horses and burros in each managed HMA and HA on March 1 of each year. Here, "abundance" refers to the number of animals present in a surveyed area, most often associated with one HMA or a complex of multiple HMAs. Some people refer to this as "herd size" for a given HMA or HA. We prefer use of the term abundance instead of the more commonly used "population size," because some definitions of "population" refer to a larger set of demographically or genetically interacting animals than is a present in a single HMA or complex. A given wild horse or burro herd may use lands across many HMAs and HAs managed as a complex, but BLM reports annual abundance at the level of HMAs and HAs.

In most cases, aerial surveys represent the best methods for estimation of abundance at the time of survey. In some cases, a gather operation after an aerial survey will provide a more recent approximation of the abundance, taking into account a surveybased estimate, the known numbers of animals removed, and the known numbers of animals returned to the range. However, several months or years may transpire between the time of those surveys or gathers and the annual March 1 reporting date. Abundance projections should account for the most reliable values for past abundance, estimates of annual population growth rates, and recorded numbers of animals removed at different times.

In the context of this SOP, we define an "estimate" as the expected value of a given population size or growth rate based on the survey data and analysis. Hence, an estimate is not a guess. Estimates have associated confidence intervals around them to acknowledge that there is uncertainty in the estimate, but to also bracket that uncertainty quantitatively. In this SOP, we denote estimates of abundance with the "hat" symbol; for example, $N$ is the symbol for true abundance and $\hat{N}$ symbolizes the estimate of abundance.

Abundance estimates from aerial surveys analyzed with methods to explicitly estimate the number of animals not detected (detection bias) could be contrasted with abundance values coming from direct count aerial surveys, aerial surveys that apply a constant correction factor with an assumed level of detection bias, from expert opinion, or from informed guesses. In this SOP, we denote informed guesses of abundance with the "dot" symbol, for example $\dot{N}$ would symbolize an informed guess of abundance based on a direct count and guessed correction factor. Following standard nomenclature in population dynamics models we symbolize annual growth rate with $\lambda$, and we contrast population growth rate estimates $(\hat{\lambda})$ with informed guesses $(\dot{\lambda})$. Note that $\lambda$, the true annual population growth rate, is a concept since we often cannot directly observe it. In practice, $\lambda$ represents our expectation of the year-to-year growth rate of a population. $\lambda$ is multiplicative, so a value above 1.0 indicates a growing population and a value below 1.0 indicates a declining population. A value of 1.2 is the same as a 20 percent per year annual increase in abundance; a value of 1.05 means a 5 percent per year increase.

\section{Approximating the Annual Growth Rate, lambda $(\lambda)$}

Population growth rate is an extremely important parameter for projections of estimated abundance of both horses and burros. Unfortunately, we have an incomplete understanding of the average expected annual growth rate for most HMAs and HAs. We have even less knowledge of how annual growth rates respond to such factors as annual variation in climate, the relative density of horses and burros, differences in sex- and age- structure, and other stochastic (random) factors. Here we demonstrate both how to calculate estimates of $\lambda$ and how to apply those estimates to predict future horse and burro abundance.

The number of adults present on March 1 should be multiplied by $\lambda$ to find the number of adults that we expect to be present in that same population one year later. Here, "Adults" are taken to include yearlings that were born in the previous foaling season. Since we are assuming foals from each year have not yet been born by March 1 (foaling season is generally assumed to be March 1 to August 31 although foals may be born after that period), ours is a very simple form of a "pre-birth pulse" population model. Population models that take into account the age distribution, sex distribution, and age-specific reproductive and survival rates for a given population may reflect the most realistic expectations of population change from year to year. Such detailed data are not available for most HMAs, though it would be welcomed. 
In this SOP, we rely on the simpler $\lambda$ for the population projections presented. As much as possible, BLM is moving toward obtaining estimates of abundance from surveys, by using survey methods that account for and estimate the number of horses present, but not seen by any observer, and also that lead to estimates of uncertainty (confidence intervals) around the abundance estimate. Reliable estimates of $\lambda$, though, have been made for only a small number of HMAs (for example, Garrott and others, 1991), and even those estimates are for past rates of population growth that may not be applicable under current conditions of population density, resource condition, and prevalence of contraception-treated animals. For abundance projections detailed below, uncertainty around the appropriate value of $\lambda$ for each HMA can reduce the reliability of future abundance estimates, even if current herd size estimates from aerial surveys are precise and accurate.

Where possible, managers should use published estimates of $\hat{\lambda}$ for the complexes, HMAs and HAs in which those estimates apply. In areas with two or more aerial surveys with reliable estimates of abundance available, it is possible to estimate the realized annual growth rate for that population over that time period. Calculating $\lambda$ from two surveys conducted $x$ years apart can be done by taking the $x$ th root of the ratio of the two abundance estimates. For example, if the estimated abundance in year $t$

was $\hat{N}_{t}$, and the estimate $x$ years later was $\hat{N}_{t+x}$, and assuming that there were no animals removed by gathers in the time interval between those two surveys, then:

$$
\hat{\lambda}=\sqrt[x]{\frac{\hat{N}_{t+x}}{\hat{N}_{t}}},
$$

where:

$\hat{\lambda} \quad$ is the annual growth rate estimate,

$\hat{N} \quad$ is an estimate of abundance, and

$t$ is the year.

In cases where there are three or more reliable surveys with no removals occurring in the time interval spanned by those three surveys, then $\hat{\lambda}$ can be estimated as the slope of a linear regression line fit to the log-transformed estimates of the abundance values (Caughley, 1977). Surveys can be weighted according to the inverse of their uncertainty, such that more precise surveys get weighted more than less precise surveys (Gerrodette, 1991). Analysis with this type of linear regression can also provide estimates of uncertainty (that is, standard errors, or confidence intervals) for $\hat{\lambda}$.

In most managed herds, horses may have been gathered and removed at various times between successive surveys. In this SOP, we do not go into detail about the methods that are appropriate for estimating $\lambda$ in those circumstances, except to mention that models to estimate $\lambda$ in those cases must account for both annual growth and the known numbers of animals removed at specific times. The goal for such analyses remains to estimate an applicable value for annual growth rate, $\lambda$, that can be applied to the March 1 herd size estimate of horses or burros to find the expected size of that population one year later. We acknowledge that projecting future population growth using estimates of $\lambda$ based on past growth rates reflects an assumption that past growth rates are applicable in the future. Unless a herd has started to receive some type of fertility control treatments, or there has been some other major change in factors that would influence growth rate, this assumption seems appropriate for most wild horse and burro populations.

For most wild horse or burro herds, there might be no local estimate of $\lambda$ available until there have been two or more reliable aerial surveys of the population. Until herd-specific estimates of $\lambda$ are available for a given population, it is reasonable to use values of $\lambda$ that are considered best approximations by expert opinion (for example, the local WHB Specialist, state lead, and other sources).

\section{Projecting Future Abundance}

The methods used to project future abundance depend on the available data about the herd. In preparation for projecting the estimated abundance at year $t+1$, we define five types of information sources for the previous year's reported abundance.

1. Abundance values for the previous March 1, whether they be estimates or informed guesses;

2. Survey information from a direct count, with or without application of a fixed ( $a d$ hoc, that is, best guess) correction factor;

3. Post-gather, expert opinion of the number of animals present;

4. Aerial surveys leading to abundance estimate that accounts for unseen animals and includes an estimate of uncertainty; and

5. Detailed information about animals that were removed from the herd, including the numbers of adults and foals removed, and the approximate removal dates. 
For cases 2,3, and 4, the time of year when the count, gather, or survey took place will influence the choice about whether or not to multiply the population value by $\lambda$. Figure 21 demonstrates how to use this information in cases where there has been no recent gather. We project future abundance values based on only adults observed if the information source was during a time period when there was still a substantial percentage of the annual foal production yet to have been born. In contrast, if the information source was from a time of year after almost all foals have been born, then we project future abundance values based on the total estimated number of adults and foals in the area at the time of data collection.

For example, if the information source was an aerial survey that took place in June, the abundance projections for the following year will multiply $\lambda$ by the estimated number of adults present, because an unknown fraction of the foal "crop" was yet to be born in June. In such a case, multiplying $\lambda$ by the estimated number of adults in the population will account for the number of foals that would be expected to be born and survive through February 28 of the next year. However, if the information source was an aerial survey that took place in September or later, we assume that the great majority of foals that would be born in that year would have already been born, and we would use both the estimated adult and estimated foal populations to develop an estimate for the total abundance. In either case, the herd size projections we use are simplifications that overlook some aspects of adult and foal survival, because monthly survival rates are unknown for most if not all HMAs. As detailed in SOP 1 Conducting Aerial Surveys with the Simultaneous Double-Observer Method, animals that are recorded as "adults" include both yearlings and older adults, while "foals" are the young animals born in the year of the survey. More details on the herd size projection method for each type of information source and time of year are below, and implications of the assumptions behind these methods follow. In all cases, the herd size projections will be strongly influenced by the choice of approximate annual population growth rate, $\lambda$.

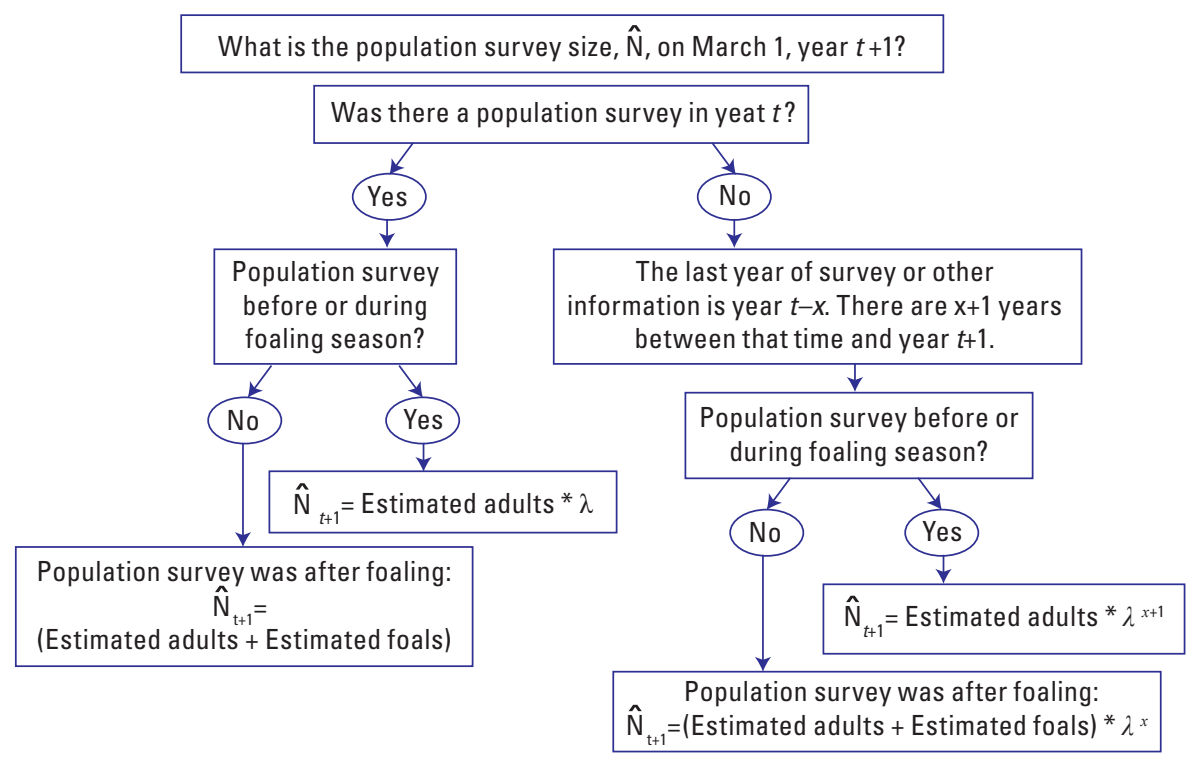

Figure 21. Projecting population size for wild horses and burros using a flow chart that diagrams the choices made in to determine the estimated abundance, $\hat{N}$, on March 1, of year $t+1$, for herds with no recent gather. Choices about whether to multiply an abundance estimate from year $t$ by the annual growth rate, $\lambda$, depend on whether the survey or other information source was at a time of year after the great majority of foaling was expected to have already taken place (that is, after August 31$)$. $(\hat{N}$, estimate of abundance; $\lambda$, annual growth rate; $t$, year) 


\section{Simplest Population Projections}

The simplest population projections are applied to cases where the available information on abundance applicable to an HMA is the value for total number of animals that was reported for the preceding year. If the preceding year is considered year $t$, that herd size could be abbreviated as $N_{t}$, and the estimated abundance for the next year can be abbreviated as $N_{t+1}$. In these cases, the herd size reported for the subsequent year is simply the annual growth rate times the previous year's estimate of abundance, or

$$
N_{t+1}=\lambda \times N_{t},
$$

where:

$N \quad$ is the reported abundance, or expected abundance;

$t \quad$ is the year; and

$\lambda \quad$ is the annual growth rate.

\section{Projections from Population Surveys that Account for Animals Missed}

BLM uses aerial survey methods that explicitly account for the number of animals not seen by any observer (see BLM Instructional Memorandum 2010-057 [BLM, 2010]). The SOPs in this document represent one type of such survey, using the double-observer method. These lead to herd size estimates for adults and foals, and associated uncertainty in the form of confidence intervals. If the post-gather population values are for a month that is during the foaling season, then the abundance on the following March 1 is assumed to be:

$$
\hat{N}_{t+1}=\hat{N}_{\text {tadults }} \times \lambda,
$$

where:

$\hat{N} \quad$ is the estimate of abundance,

$t \quad$ is the year, and

$\lambda \quad$ is the annual growth rate.

This reflects an assumption that only a negligible number of adults have died between the time of the count and the previous March 1. Given the generally high survival rates of adult horses in most herds for which detailed demographic rates have been reported, that is usually a reasonable assumption for the purposes of simple projections.

If the survey abundance estimates are from a month that is not during the foaling season (that is, assumed to be from September 1 to February 28), then the abundance on the following March 1 is assumed to be:

$$
\hat{N}_{t+1}=\hat{N}_{t, \text { adults }}+\hat{N}_{t, \text { foals }},
$$

where:

$$
\begin{aligned}
\hat{N} & \text { is an estimate of abundance, and } \\
t & \text { is the year. }
\end{aligned}
$$

For every 1 additional year that elapses, the abundance on March 1 is multiplied again by $\lambda$ to find the approximate abundance on the following March 1.

Estimated abundance values that are associated with confidence intervals can allow for projection of 90 percent confidence intervals of future abundance. Unless there is information concerning the variance around the annual growth rate $\lambda$, 90 percent confidence intervals for abundance on March 1 are determined as above, except that the applicable values of $N_{0, \text { adults }}$ and $N_{0, \text { foals }}$ are taken from the 90 percent lower confidence limit and 90 percent upper confidence limit for those values. If there are estimates for the variance of $\hat{\lambda}$, then that information can be incorporated into projections, using the delta method for variance approximation (Seber, 1982). 


\section{Projections from Direct Counts}

Direct counts from aerial surveys typically result in a raw count of the number of animals seen, which may also be called a "tally" or simply a "count." This will usually be a count of adults and foals. In certain cases, the specialist might apply an ad hoc (best guess) fixed correction factor that is assumed to account for the animals that were present in the HMA and associated area, but not seen in the survey, but where the magnitude of the correction factor is not based on any statistical analysis of the patterns of detection in the survey data. The survey and ad hoc correction factor result in an approximation of the number of adults, $\dot{N}_{t, \text { adults }}$ and foals, $\dot{N}_{t, \text { foals }}$, in the area at the time of the survey. The month and year of the survey are known. If the survey took place during the foaling season (generally assumed to be March 1 to August 31), then the value for abundance on the following March 1 is assumed to be:

$$
\dot{N}_{t+1}=\dot{N}_{t, \text { adults }} \times \lambda,
$$

where:

$\dot{N} \quad$ is the informed guess of abundance,

$t \quad$ is the year, and

$\lambda \quad$ is the annual growth rate.

This type of projection is based on assumptions that the values for $\dot{N}_{t}$ and $\lambda$ are reliable. Also, this reflects an assumption that only a negligible number of adults have died between the time of the count and the previous March 1.

If the survey took place after the end of the assumed foaling season (that is, between September 1 to February 28), then the value for herd size on the following March 1 is assumed to be:

$$
\dot{N}_{t+1}=\dot{N}_{t, \text { adults }}+\dot{N}_{t, \text { foals }}
$$

where:

$\dot{N} \quad$ is an informed guess of abundance, and

$t$ is the year.

After the herd size is projected for $\dot{N}_{t^{+1}}$ of March 1, then for every 1 additional year that elapses, the herd size on March 1 is simply multiplied by $\lambda$ to find the approximate herd size on the following March 1 .

\section{Projections from Post-gather Expert Opinion of the Number of Animals}

Generally, after a gather the specialist will have a reasonable estimate of the number of adults and foals that are present in the herd that uses an HMA. This is because the specialist will usually have an approximate value, or an estimate of the number of horses and burros present in an HMA before the gather takes place. Then, the specialist knows exactly how many horses and burros are removed and how many are turned back to the range after a gather. Therefore, the specialist can simply subtract the number of adults and foals removed from the pre-gather population estimate to find the estimated number of animals that remain. That number might also be partially confirmed by a post-gather reconnaissance flight.

The post-gather estimated abundance of adults and foals is associated with a month and year. As was the case for direct counts, if the post-gather abundance values are for a month that is during the foaling season (generally assumed to be March 1 to August 31, although foals may be born outside of that period), then the estimated abundance on the following March 1 is assumed to be:

$$
\hat{N}_{t+1}=\hat{N}_{t, a d u l t s} \times \lambda
$$

where:

$\hat{N} \quad$ is an estimate of abundance,

$t \quad$ is the year, and

$\lambda \quad$ is the annual growth rate.

If the post-gather abundance values are from a month outside of the foaling season (that is, between September 1 and February 28), then the abundance on the following March 1 is assumed to be:

$$
\hat{N}_{t+1}=\hat{N}_{t, \text { adults }}+\hat{N}_{t, \text { foals }} \text {, }
$$

where:

$\hat{N} \quad$ is an estimate of abundance, and

$t$ is the year.

For every 1 additional year that elapses, the estimated abundance on March 1 is multiplied again by $\lambda$ to find the approximate abundance on the following March 1. 


\section{Caveats About the Methods Above}

As noted previously, herd size projections are directly proportional to the assumed values of $\lambda$ applied to each population. Unless these assumed values are based on empirical studies from the population of interest, there can be an unquantified degree of uncertainty associated with the assumed values.

Monthly adult and foal survival rates have a less straightforward effect on population projections. We recognize that the approach outlined above is a simplification of complex demographic processes. The single annual growth rate, $\lambda$, applicable from March 1 of one year to March 1 of the next year, is actually the result of several vital rates, including annual stallion survival rates, annual yearling survival rate, mare fertility rate, mare survival rate to foaling, mare survival rate past foaling, and foal survival rate. Modeling these rates in a way that accurately accounts for the intricacies of changing age-and sex-structure in each population should make use of matrix population models (Garrott and others, 1991; Caswell, 2000). Unfortunately, for the vast majority of HMAs, HAs, and WHTs, few of the requisite rates are known accurately and precisely enough to make matrix population projections meaningful. As such, we generally fall back to the simpler application of an annual growth rate.

If anything, the projected abundance values from the approach outlined above will tend to lead to slight underestimates of true population size for cases where the information source is from time periods between March 1 and August 31. This is because some number of adults may have died from March 1 up to the time when the surveys took place. As noted above, in cases where the information source was from the time period between March 1 and August 31, the subsequent March 1 population (for year $t+1$ ) is taken to be $\lambda$ times the estimated number of adults at the time of the survey or other information source. The application of $\lambda$ to the slightly decreased adult abundance (relative to the adult population on March 1) will lead to an abundance estimate for the subsequent year that is lower than it would have been if $\lambda$ had been multiplied by the March 1 population (for year $t$ ). In most populations and for most years, this will tend to cause only a negligible bias in future population projections, because adult and yearling annual survival rates tend to be high, often with 90 percent or more for adults (Goodloe and others, 2000; Turner and Morrison, 2001; Grange and others, 2009, Scorolli and Lopez Cazorla, 2010) and often above 60-80 percent for foals and yearlings (Dawson, 2005; Grange and others, 2009; Scorolli and Lopez Cazorla, 2010) unless there is high predation on foals (for example, Turner and Morrison, 2001), which is generally rare.

Conversely, the projected abundance values from the approach outlined above could lead to slight overestimates of true population size in certain circumstances when the information source is from a time period between September 1 and February 28. As noted above, in such cases, the abundance value reported for March 1 is taken to be the estimated total number of adults and the previous year's foals. This reflects assumptions that few or no further foals will be born, and that there will be little foal or adult mortality until March 1. In practice, there will probably not be too many cases where this causes much of an overestimation, first because some small number of foals may continue to be born after September 1, and second because foal overwinter survival tends to be fairly high in most studied populations (for example, Dawson, 2005). Moreover, any tendency that counting all foals toward the subsequent March 1 abundance value will bias that estimate high may be somewhat counteracted by adult mortality that may have occurred since the preceding March 1.

The methods presented above are simplifications of actual horse and burro population dynamics. Nonetheless, having these methods documented allows for transparency in explaining the calculations that can lead to estimated abundance values for each HMA, and will clarify which HMAs or complexes are in the greatest need of future surveys and estimates of realized annual growth rates.

\section{References Cited}

Bureau of Land Management (BLM), 2010, Wild horse and burro population inventory and estimation: Bureau of Land Management, Instruction Memorandum 2010-057. [Also available at https://www.blm.gov/policy/im-2010-057.]

Caswell, H., 2000, Matrix population models-Construction, analysis, and Interpretation ( $2 \mathrm{~d}$ ed.): Sunderland, Mass., Sinauer Associates, Inc., 722 p.

Caughley, G., 1977, Analysis of vertebrate populations: New York, John Wiley and Sons, $234 \mathrm{p}$.
Dawson, M., 2005, The population ecology of feral horses in the Australian Alps management summary: Prepared for the Australian Alps Liaison Committee, April 2005. [Also available at http://www.australianalps.environment.gov.au/ publications/research-reports/pubs/feral-horses.pdf.]

Garrott, R.A., Siniff, D.B., and Eberhardt, L.L., 1991, Growth rates of feral horse populations: Journal of Wildlife Management v. 55, no. 4, p. 641-648. [Also available at https://doi.org/10.2307/3809513.]

Gerrodette, T., 1991, Models for power of detecting trends-A reply to Link and Hatfield: Ecology, v. 72, no. 5, p.18891892. [Also available at https://doi.org/10.2307/1940986.] 
Goodloe, R.B., Warren, R.J., Osborn, D.A., and Hall, C., 2000, Population characteristics of feral horses on Cumberland Island, Georgia and their management implications: Journal of Wildlife Management, v. 64, no. 1, p. 114-121. [Also available at https://doi.org/10.2307/3802980.]

Grange, S., Duncan, P., and Gaillard, J-M., 2009, Poor horse traders - Large mammals trade survival for reproduction during the process of feralization in Proceedings: The Royal Society B, Biological Sciences, v. 276, issue 1663, p. 1911-1919. [Also available at https://doi.org/10.1098/ rspb.2008.1828.]

Scorolli, A.L., and Lopez Cazorla, A. C., 2010, Demography of feral horses (Equus caballus) - A long-term study in Tornquist Park, Argentina: Wildlife Research, v. 37, no. 3, p. 207-214. [Also available at https://doi.org/10.1071/ WR09059.]

Seber, G.A.F., 1982, The estimation of animal abundance and related parameters ( $2 \mathrm{~d}$ ed.): New York, Macmillan, $672 \mathrm{p}$.

Turner, J.W., and Morrison, M.L., 2001, Influence of predation by mountain lions on numbers and survivorship of a feral horse population: The Southwestern Naturalist, v. 46, no. 2, p. 183-190. [Also available at https://doi.org/10.2307/3672527.] 


\section{Appendix 1. List of Herd Codes, by State}

\section{Bureau of Land Management Herd Management Areas and Herd Areas}

Table 1.1. This is a list of two-letter, four-digit herd codes used for Bureau of Land Management Herd Management Areas (HMAs) and Herd Areas (HAs), current as of November 2019.

\begin{tabular}{|c|c|}
\hline Herd area name & Herd code \\
\hline \multicolumn{2}{|c|}{ Arizona HMAs } \\
\hline Alamo & AZ0005 \\
\hline Big Sandy & AZ0004 \\
\hline Black Mountain & AZ0003 \\
\hline Cerbat Mountains & AZ0002 \\
\hline Cibola-Trigo & AZ0011 \\
\hline Havasu & AZ0010 \\
\hline Lake Pleasant & AZ0006 \\
\hline \multicolumn{2}{|c|}{ Arizona HAs } \\
\hline Harquahala & AZ0008 \\
\hline Little Harquahala Mountains & AZ0009 \\
\hline Painted Rocks & AZ0007 \\
\hline Tassi-Gold Butte & AZ0001 \\
\hline Outside Of Herd Areas & AZ000Z \\
\hline \multicolumn{2}{|c|}{ California HMAs } \\
\hline Bitner (from CA0268) & CA0267 \\
\hline Buckhorn (from CA0270) & CA0262 \\
\hline Carter Reservoir (from CA0260) & CA0269 \\
\hline Centennial & CA0654 \\
\hline Chemehuevi & CA0698 \\
\hline Chicago Valley & CA0681 \\
\hline Chocolate-Mule Mountains & CA0671 \\
\hline Coppersmith (from CA0270) & CA0261 \\
\hline Fort Sage & CA0241 \\
\hline Fox Hog & CA0263 \\
\hline High Rock & CA0264 \\
\hline Lee Flat & CA0652 \\
\hline Massacre Lakes & CA0268 \\
\hline New Ravendale & CA0243 \\
\hline Nut Mountain (from CA0264 and CA0268) & CA0266 \\
\hline Piper Mountain & CA0656 \\
\hline Red Rock Lakes & CA0251 \\
\hline Twin Peaks & CA0242 \\
\hline Wall Canyon (from CA0264) & CA0265 \\
\hline Waucoba-Hunter Mountain & CA0651 \\
\hline Round Mountain (Devils Garden Plateau) & CA0252 \\
\hline
\end{tabular}


Table 1.1. This is a list of two-letter, four-digit herd codes used for Bureau of Land Management Herd Management Areas (HMAs) and Herd Areas (HAs), current as of November 2019.-Continued

\begin{tabular}{|c|c|}
\hline Herd area name & Herd code \\
\hline \multicolumn{2}{|c|}{ California HAs } \\
\hline Cima Dome & CA0695 \\
\hline Clark Mountain & CA0691 \\
\hline Coyote Canyon & CA0663 \\
\hline Dead Mountain & CA0697 \\
\hline Granite-Providence Mountains & CA0693 \\
\hline Kramer & CA0682 \\
\hline Lava Beds & CA0692 \\
\hline Morongo & CA0661 \\
\hline New Years Lake (into CA0269) & CA0260 \\
\hline Palm Canyon & CA0662 \\
\hline Panamint & CA0653 \\
\hline Picacho (within CA0671) & CA0672 \\
\hline Piute Mountain & CA0696 \\
\hline Sand Spring-Last Chance & CA0657 \\
\hline Slate Range & CA0655 \\
\hline Tuledad (in CA0261 and CA0262) & CA0270 \\
\hline Woods-Hackberry & CA0694 \\
\hline Outside Of Herd Areas & CA000Z \\
\hline \multicolumn{2}{|c|}{ Colorado HMAs } \\
\hline Little Book Cliffs Wild Horse Range & $\mathrm{CO} 0766$ \\
\hline Piceance-East Douglas Creek & CO0161 \\
\hline Sand Wash Basin & $\mathrm{CO} 0143$ \\
\hline Spring Creek Basin & CO0384 \\
\hline \multicolumn{2}{|c|}{ Colorado HAs } \\
\hline Douglas Mountain & $\mathrm{CO} 0144$ \\
\hline Naturita Ridge & $\mathrm{CO} 0345$ \\
\hline North Piceance (in CO0161) & $\mathrm{CO} 0163$ \\
\hline West Douglas Creek & CO0162 \\
\hline Outside of Herd Areas & $\mathrm{CO} 000 \mathrm{Z}$ \\
\hline \multicolumn{2}{|c|}{ Idaho HMAs } \\
\hline Black Mountain & ID0006 \\
\hline Challis & ID0005 \\
\hline Four Mile & ID0002 \\
\hline Hard Trigger & ID0007 \\
\hline Sand Basin & ID0004 \\
\hline Saylor Creek & ID0003 \\
\hline \multicolumn{2}{|c|}{ Idaho HAs } \\
\hline Morgan Creek & ID0009 \\
\hline Sheep Mountain & ID0008 \\
\hline West Crane Creek & ID0001 \\
\hline Outside Of Herd Areas & ID000Z \\
\hline Diamond (all claimed-not mapped) & ID0010 \\
\hline
\end{tabular}


Table 1.1. This is a list of two-letter, four-digit herd codes used for Bureau of Land Management Herd Management Areas (HMAs) and Herd Areas (HAs), current as of November 2019.-Continued

\begin{tabular}{|c|c|}
\hline Herd area name & Herd code \\
\hline \multicolumn{2}{|l|}{ Montana HMAs } \\
\hline Pryor Mountain Wild Horse Range & MT0251 \\
\hline \multicolumn{2}{|l|}{ Montana HAs } \\
\hline Barton Gulch & MT0253 \\
\hline Devils Fence & MT0254 \\
\hline Ervin Ridge & МT0252 \\
\hline Garden Creek & MT0255 \\
\hline Rocky Hills & MT0257 \\
\hline Sweetwater & МT0256 \\
\hline Outside of Herd Areas & MT000Z \\
\hline \multicolumn{2}{|l|}{ Nevada HMAs } \\
\hline Amargosa Valley & NV0511 \\
\hline Antelope (from NV0490 and NV0495) & NV0401 \\
\hline Antelope Valley (from NV0106) & NV0107 \\
\hline Ash Meadows & NV0509 \\
\hline Augusta Mountains & NV0311 \\
\hline Bald Mountain & NV0603 \\
\hline $\begin{array}{l}\text { Black Rock Range (combination of Black Rock Range and Black } \\
\text { Rock Range West) }\end{array}$ & NV0234 \\
\hline Blue Wing Mountains & NV0217 \\
\hline Buffalo Hills & NV0220 \\
\hline Bullfrog & NV0629 \\
\hline Calico Mountains & NV0222 \\
\hline Callaghan (from NV0687 and NV0689) & NV0604 \\
\hline Clan Alpine & NV0310 \\
\hline Desatoya (from NV0688) & NV0606 \\
\hline Diamond & NV0609 \\
\hline Diamond Hills North & NV0104 \\
\hline Diamond Hills South (original name Railroad Pass) & NV0412 \\
\hline Dogskin Mountains & NV0302 \\
\hline Eagle (from NV0404 and NV0521) & NV0414 \\
\hline El Dorado Mountains & NV0501 \\
\hline Fish Creek (from NV0698) & NV0612 \\
\hline Fish Lake Valley & NV0622 \\
\hline Flanigan & NV0301 \\
\hline Fort Sage & NV0320 \\
\hline Fox-Lake Range & NV0228 \\
\hline Garfield Flat & NV0313 \\
\hline Gold Butte & NV0502 \\
\hline Gold Mountain & NV0628 \\
\hline Goldfield & NV0626 \\
\hline Goshute & NV0108 \\
\hline Granite Peak & NV0303 \\
\hline Granite Range & NV0221 \\
\hline
\end{tabular}




\section{Standard Operating Procedures for Wild Horse and Burro Double-Observer Aerial Surveys}

Table 1.1. This is a list of two-letter, four-digit herd codes used for Bureau of Land Management Herd Management Areas (HMAs) and Herd Areas (HAs), current as of November 2019.-Continued

\begin{tabular}{|c|c|}
\hline Herd area name & Herd code \\
\hline Horse Mountain & NV0308 \\
\hline Hot Creek & NV0616 \\
\hline Jackson Mountains & NV0208 \\
\hline Johnnie (from NV0527 and NV0508) & NV0510 \\
\hline Kamma Mountains & NV0214 \\
\hline Lahontan & NV0306 \\
\hline Lava Beds & NV0215 \\
\hline Little Fish Lake & NV0614 \\
\hline Little Humboldt & NV0102 \\
\hline Little Owyhee & NV0200 \\
\hline Marietta Wild Burro Range & NV0316 \\
\hline Maverick-Medicine (from NV0106) & NV0105 \\
\hline Mcgee Mountian & NV0210 \\
\hline Montezuma Peak & NV0625 \\
\hline Muddy Mountains & NV0503 \\
\hline Nevada Wild Horse Range & NV0524 \\
\hline New Pass-Ravenswood & NV0602 \\
\hline Nightengale Mountains & NV0219 \\
\hline North Stillwater & NV0229 \\
\hline Owyhee & NV0101 \\
\hline Palmetto & NV0624 \\
\hline Pancake (from NV0402 and NV0405) & NV0415 \\
\hline Paymaster (original name Paymaster-Lone Mountain) & NV0621 \\
\hline Pilot Mountain (from NV0315 and NV0690) & NV0314 \\
\hline Pine Nut Mountains & NV0305 \\
\hline Red Rock (from NV0525) (original name Red Rock-Bird Springs) & NV0504 \\
\hline Reveille & NV0619 \\
\hline Roberts Mountain & NV0607 \\
\hline Rock Creek & NV0103 \\
\hline Rocky Hills (original name North Simpson Park) & NV0605 \\
\hline Sand Springs West & NV0630 \\
\hline Saulsbury & NV0620 \\
\hline Seven Mile (original name Antelope (HA)) & NV0613 \\
\hline Seven Troughs & NV0216 \\
\hline Shawave Mountains & NV0218 \\
\hline Silver King (from NV0410, NV0522, and NV0523) & NV0416 \\
\hline Silver Peak & NV0623 \\
\hline Snowstorm Mountains & NV0201 \\
\hline South Shoshone & NV0601 \\
\hline South Stillwater & NV0309 \\
\hline Spruce-Pequop & NV0109 \\
\hline Stone Cabin (from NV0699) & NV0618 \\
\hline Stonewall & NV0627 \\
\hline Tobin Range & NV0231 \\
\hline
\end{tabular}


Table 1.1. This is a list of two-letter, four-digit herd codes used for Bureau of Land Management Herd Management Areas (HMAs) and Herd Areas (HAs), current as of November 2019.-Continued

\begin{tabular}{|c|c|}
\hline Herd area name & Herd code \\
\hline Triple B (from NV0403, NV0407, and NV0406) & NV0417 \\
\hline Warm Springs Canyon & NV0226 \\
\hline Wassuk & NV0312 \\
\hline Wheeler Pass (from NV0525) & NV0507 \\
\hline Whistler Mountain (from NV0698) & NV0608 \\
\hline Hickison Summit & NV0610 \\
\hline Montgomery Pass & NV0317 \\
\hline North Monitor & NV0611 \\
\hline \multicolumn{2}{|l|}{ Nevada HAs } \\
\hline Antelope Range & NV0211 \\
\hline Applewhite & NV0518 \\
\hline Austin (into NV0604) & NV0687 \\
\hline Bloody Runs & NV0204 \\
\hline Blue Diamond (in NV0525) & NV0505 \\
\hline Blue Nose Peak & NV0514 \\
\hline Buck-Bald (into NV0417) & NV0403 \\
\hline Bulter Basin & NV0686 \\
\hline Butte (from NV0406; Into NV0417) & NV0407 \\
\hline Cave Valley (in NV0410) & NV0493 \\
\hline Cherry Creek (into NV0417) (original name Cherry Creek-Egan) & NV0406 \\
\hline Clover Creek & NV0517 \\
\hline Clover Mountains & NV0516 \\
\hline Deer Lodge Canyon (into NV0414) & NV0521 \\
\hline Delamar Mountains & NV0515 \\
\hline Dobbin Summit & NV0615 \\
\hline Dry Lake (into NV0416) & NV0410 \\
\hline Dunlap (into NV0314) & NV0690 \\
\hline East Range & NV0225 \\
\hline Eugene Mountains & NV0207 \\
\hline Fortification (into NV0404) & NV0496 \\
\hline Gabbs Valley Range (into NV314) & NV0315 \\
\hline Golden Gate (into NV0411) & NV0494 \\
\hline Goshute (in NV0401) & NV0495 \\
\hline Grass Valley (into NV0604) & NV0689 \\
\hline Highland Peak (into NV0416) & NV0522 \\
\hline Horse Spring & NV0307 \\
\hline Hot Springs Mountains & NV0203 \\
\hline Humboldt & NV0224 \\
\hline Ione & NV0691 \\
\hline Jakes Wash & NV0408 \\
\hline Kobeh Valley (into NV0608 and NV0612) & NV0698 \\
\hline Krum Hills & NV0206 \\
\hline Last Chance (into NV0510) & NV0527 \\
\hline Little Mountain & NV0519 \\
\hline
\end{tabular}


Table 1.1. This is a list of two-letter, four-digit herd codes used for Bureau of Land Management Herd Management Areas (HMAs) and Herd Areas (HAs), current as of November 2019.-Continued

\begin{tabular}{|c|c|}
\hline Herd area name & Herd code \\
\hline Lower Paradise Valley & NV0233 \\
\hline Lucky Strike (in NV0525) & NV0526 \\
\hline Mavrick-Butte (into NV0403) & NV0491 \\
\hline Meadow Valley Mountains & NV0513 \\
\hline Miller Flat & NV0520 \\
\hline Monte Cristo (into NV0415) & NV0402 \\
\hline Moriah & NV0413 \\
\hline Mormon Mountains & NV0512 \\
\hline Mount Airy & NV0695 \\
\hline Mount Potosi (in NV0525) & NV0506 \\
\hline Mount Sterling (into NV0510) & NV0508 \\
\hline Murphys Wash & NV0452 \\
\hline North Cherry Creek (in NV0105 and NV0107) & NV0106 \\
\hline North Shoshone & NV0697 \\
\hline Osgood Mountains & NV0202 \\
\hline Pah Rah Mountains & NV0304 \\
\hline Park Mountain & NV0694 \\
\hline Patterson-Eagle (into NV0404) & NV0492 \\
\hline Powell Mountain & NV0318 \\
\hline Quinn & NV0696 \\
\hline Rattlesnake (into NV0416) & NV0523 \\
\hline Sand Springs East (into NV0415) & NV0405 \\
\hline Schell Creek (into NV0401) & NV0490 \\
\hline Seaman Range & NV0411 \\
\hline Selenite Range & NV0212 \\
\hline Slumbering Hills & NV0205 \\
\hline Smith Creek (into NV0606) & NV0688 \\
\hline Sonoma Range & NV0223 \\
\hline South Pancake & NV0692 \\
\hline South Slumbering Hills & NV0230 \\
\hline Spring Mountain (in NV0504 And Nv0507) & NV0525 \\
\hline Toano & NV0110 \\
\hline Trinity Range & NV0232 \\
\hline Truckee Range & NV0213 \\
\hline Tule Ridge/Mahogany Flat & NV0319 \\
\hline Upper Hot Creek & NV0693 \\
\hline White River & NV0409 \\
\hline Willow Creek (in NV0618) & NV0699 \\
\hline Wilson Creek (from into NV0414) & NV0404 \\
\hline Outside Of Herd Areas & NV000Z \\
\hline \multicolumn{2}{|c|}{ New Mexico HMAs } \\
\hline
\end{tabular}

Bordo Atravesado

NM0001 
Table 1.1. This is a list of two-letter, four-digit herd codes used for Bureau of Land Management Herd Management Areas (HMAs) and Herd Areas (HAs), current as of November 2019.—Continued

\begin{tabular}{|c|c|}
\hline Herd area name & Herd code \\
\hline \multicolumn{2}{|c|}{ New Mexico HAs } \\
\hline Carracas Mesa (original name Rosa Community) & NM0003 \\
\hline Godfrey Hills & NM0002 \\
\hline Punche Valley & NM0004 \\
\hline Outside Of Herd Areas & NM000Z \\
\hline \multicolumn{2}{|c|}{ Oregon HMAs } \\
\hline Beatys Butte & OR0002 \\
\hline Cold Spring & OR0013 \\
\hline Coyote Lake/Alvord Tule Springs & OR0014 \\
\hline Hog Creek & OR0011 \\
\hline Jackies Butte & OR0015 \\
\hline Kiger (from OR0020 and OR0038) & OR0010 \\
\hline Liggett Table & OR0037 \\
\hline Paisley Desert & OR0001 \\
\hline Palomino Buttes & OR0006 \\
\hline Pokegama & OR0018 \\
\hline Riddle Mountain & OR0009 \\
\hline Sand Springs & OR0017 \\
\hline Sheepshead/Heath Creek & OR0016 \\
\hline South Steens & OR0003 \\
\hline Stinkingwater & OR0008 \\
\hline Three Fingers & OR0012 \\
\hline Warm Springs & OR0007 \\
\hline Murderers Creek & OR0019 \\
\hline \multicolumn{2}{|c|}{ Oregon HAs } \\
\hline Alvord Tule Springs & OR0005 \\
\hline Atturbury & OR0028 \\
\hline Basque & OR0025 \\
\hline Cherry Creek & OR0021 \\
\hline Cottonwood Basin & OR0026 \\
\hline Cottonwood Creek & OR0027 \\
\hline Diamond Craters & OR0031 \\
\hline East Kiger (into OR0010) & OR0020 \\
\hline East Wagontire & OR0034 \\
\hline Heath Creek-Sheepshead (into OR0016) & OR0004 \\
\hline Lakeridge & OR0023 \\
\hline Middle Fork & OR0032 \\
\hline Morger & OR0030 \\
\hline Potholes & OR0024 \\
\hline Pueblo-Lone Mountain & OR0036 \\
\hline Rhodes Canyon & OR0022 \\
\hline Second Flat & OR0033 \\
\hline Smyth Creek (into OR0010) & OR0038 \\
\hline South Catlow & OR0035 \\
\hline
\end{tabular}


Table 1.1. This is a list of two-letter, four-digit herd codes used for Bureau of Land Management Herd Management Areas (HMAs) and Herd Areas (HAs), current as of November 2019.-Continued

\begin{tabular}{|c|c|}
\hline Herd area name & Herd code \\
\hline Stockade & OR0029 \\
\hline Outside Herd Areas & OR000Z \\
\hline \multicolumn{2}{|l|}{ Utah HMAs } \\
\hline Bible Spring & UT0440 \\
\hline Canyon Lands & UT0571 \\
\hline Cedar Mountain & UT0241 \\
\hline Chloride Canyon & UT0442 \\
\hline Choke Cherry & UT0443 \\
\hline Confusion & UT0552 \\
\hline Conger & UT0553 \\
\hline Four Mile & UT0444 \\
\hline Frisco & UT0445 \\
\hline Kingtop & UT0554 \\
\hline Mount Elinor & UT0446 \\
\hline Muddy Creek (from UT0652) & UT0651 \\
\hline North Hills & UT0447 \\
\hline Onaqui Mountain & UT0242 \\
\hline Range Creek & UT0641 \\
\hline Sinbad & UT0652 \\
\hline Sulphur & UT0448 \\
\hline Swasey & UT0555 \\
\hline Tilly Creek & UT0449 \\
\hline \multicolumn{2}{|l|}{ Utah HAs } \\
\hline Blawn Wash & UT0441 \\
\hline Bonanza & UT0851 \\
\hline Burbank & UT0551 \\
\hline Harveys Fear & UT0461 \\
\hline Hill Creek & UT0852 \\
\hline Moody-Wagon Box Mesa & UT0481 \\
\hline Oquirrh Mountain & UT0243 \\
\hline Robbers Roost & UT0653 \\
\hline Winter Ridge & UT0853 \\
\hline Big Creek & UT0244 \\
\hline Outside Herd Areas & UT000Z \\
\hline \multicolumn{2}{|l|}{ Wyoming HMAs } \\
\hline Adobe Town (from WY0042, WY0044, WY0001, and WY0038) & WY0009 \\
\hline Antelope Hills (from WY0010) & WY0031 \\
\hline Conant Creek (originally part of Lander) & WY0029 \\
\hline Crooks Mountain (from WY0033) & WY0036 \\
\hline Dishpan Butte (originally part of Lander) & WY0028 \\
\hline Divide Basin (from WY0043 and WY0038) & WY0002 \\
\hline Fifteenmile & WY0011 \\
\hline Green Mountain (from WY0033) & WY0037 \\
\hline Little Colorado (into WY0003) & WY0039 \\
\hline
\end{tabular}


Table 1.1. This is a list of two-letter, four-digit herd codes used for Bureau of Land Management Herd Management Areas (HMAs) and Herd Areas (HAs), current as of November 2019.-Continued

\begin{tabular}{ll}
\multicolumn{1}{c}{ Herd area name } & Herd code \\
\hline Lost Creek (from WY0010 and WY0033) & WY0035 \\
Mccullough Peaks & WY0012 \\
Muskrat Basin (originally part of Lander) & WY0027 \\
Rock Creek Mountian (originally part of Lander) & WY0026 \\
Salt Wells Creek (from WY0038; into WY0009) & WY0001 \\
Stewart Creek (into WY0035, WY0036, WY0010, and WY0037) & WY0033 \\
$\quad$ (original name Stewart Creek/Chain Lakes) & \\
White Mountain (from WY0039 and WY0038) & WY0003 \\
\hline \multicolumn{1}{c}{ Wyoming HAs } & \\
\hline Alkali Spring Creek & WY0020 \\
Arapaho Creek & WY0034 \\
Bolten & WY0025 \\
Carter Lease & WY0017 \\
Checkerboard South & WY0040 \\
Continental Peak (into Wy0002) & WY0043 \\
Cumberland & WY0015 \\
Cyclone Rim (into WY0035, WY0033, and WY0031) & WY0010 \\
Deer Creek & WY0024 \\
Desert (original name South Desert/Figure Four) & WY0004 \\
Doty Mountain/Cherokee & WY0032 \\
East Beaver & WY0030 \\
Flat Top & WY0045 \\
Foster Gulch/Dry Creek & WY0023 \\
Gold Creek & WY0014 \\
Granger Lease (original name South Granger) & WY0005 \\
La Barge & WY0006 \\
Lander (into WY0026, WY0027, WY0028, WY0029) & WY0007 \\
North Granger & WY0018 \\
North Shoshone & WY0019 \\
Rock Springs (into WY0009, WY0003, WY0002, and WY0001) & WY0038 \\
Sand Creek East (in WY0009) & WY0042 \\
Sand Creek West (in WY0009) & WY0044 \\
Sand Draw & WY0021 \\
Seven Lakes & WY0008 \\
Slate Creek & WY0016 \\
Triangle & WY0013 \\
Outside of herd areas & WY0022 \\
\hline & WY000Z \\
\hline
\end{tabular}




\section{U.S. Forest Service Wild Horse Territories}

Table 1.2. This is a list of two-letter, four-digit herd codes used for U.S. Forest Service Wild Horse Territories (WHTs), current as of November 2019.

\begin{tabular}{|c|c|}
\hline Territory name & Herd code \\
\hline \multicolumn{2}{|c|}{ Arizona WHTs } \\
\hline Double A & AZ0021 \\
\hline Saguaro Lake & AZ0022 \\
\hline Heber & AZ0023 \\
\hline \multicolumn{2}{|c|}{ California WHTs } \\
\hline Devil/s Garden Plateau & CA0252 \\
\hline Saline Valley & CA0651 \\
\hline Brushy Mountain & CA0702 \\
\hline Black Mountain & CA0703 \\
\hline Big Bear & CA0704 \\
\hline Mcgavin Peak & CA0705 \\
\hline Three Sisters & CA0706 \\
\hline \multicolumn{2}{|c|}{ Idaho WHTs } \\
\hline Alder Creek & ID0008 \\
\hline Morgan Creek & ID0009 \\
\hline \multicolumn{2}{|c|}{ Montana WHTs } \\
\hline Pryor Mountain & MT0251 \\
\hline \multicolumn{2}{|c|}{ New Mexico WHTs } \\
\hline Jicarilla & NM0003 \\
\hline Jarita Mesa & NM0005 \\
\hline Montosa Mesa & NM0006 \\
\hline Caja Del Rio & NM0007 \\
\hline Dome & NM0008 \\
\hline San Diego & NM0009 \\
\hline Deep Creek & NM0010 \\
\hline Mesa De Las Viejas & NM0011 \\
\hline Chicoma & NM0012 \\
\hline Polvadera & NM0013 \\
\hline \multicolumn{2}{|c|}{ Nevada WHTs } \\
\hline Montgomery Pass & NV0317 \\
\hline Powell Mountain & NV0318 \\
\hline Tierney & NV0350 \\
\hline Monte Cristo & NV0402 \\
\hline Cherry Springs & NV0403 \\
\hline Jakes Wash & NV0408 \\
\hline Mt Moriah & NV0413 \\
\hline Murphy Wash & NV0452 \\
\hline Red Rock & NV0504 \\
\hline Spring Mountain & NV0507 \\
\hline
\end{tabular}

Table 1.2. This is a list of two-letter, four-digit herd codes used for U.S. Forest Service Wild Horse Territories (WHTs), current as of November 2019.-Continued

\begin{tabular}{|c|c|}
\hline Territory name & Herd code \\
\hline Johnnie & NV0510 \\
\hline Hickison Summit & NV0610 \\
\hline Kelly Creek & NV0611 \\
\hline Seven Mile & NV0613 \\
\hline Little Fish Lake & NV0614 \\
\hline Dobbin Summit & NV0615 \\
\hline Stone Cabin & NV0618 \\
\hline Monitor & NV0620 \\
\hline White Mountain & NV0622 \\
\hline Toquima & NV0652 \\
\hline Toiyabe & NV0653 \\
\hline Northumberland & NV0654 \\
\hline Paradise Peak & NV0655 \\
\hline Ellsworth & NV0656 \\
\hline Shoshone & NV0658 \\
\hline Butler Basin & NV0686 \\
\hline Quinn & NV0696 \\
\hline \multicolumn{2}{|c|}{ Oregon WHTs } \\
\hline Murderers Creek & OR0019 \\
\hline Big Summit & OR0020 \\
\hline \multicolumn{2}{|c|}{ Utah WHTs } \\
\hline Big Creek & UT0244 \\
\hline North Hills & UT0447 \\
\hline
\end{tabular}




\section{Appendix 2. Examples of Percent Concealing Vegetation, 0-80 percent}

In the examples below, the vegetation must be between hock height and head height, or higher, to be considered concealing vegetation (figure courtesy of J. Ransom).

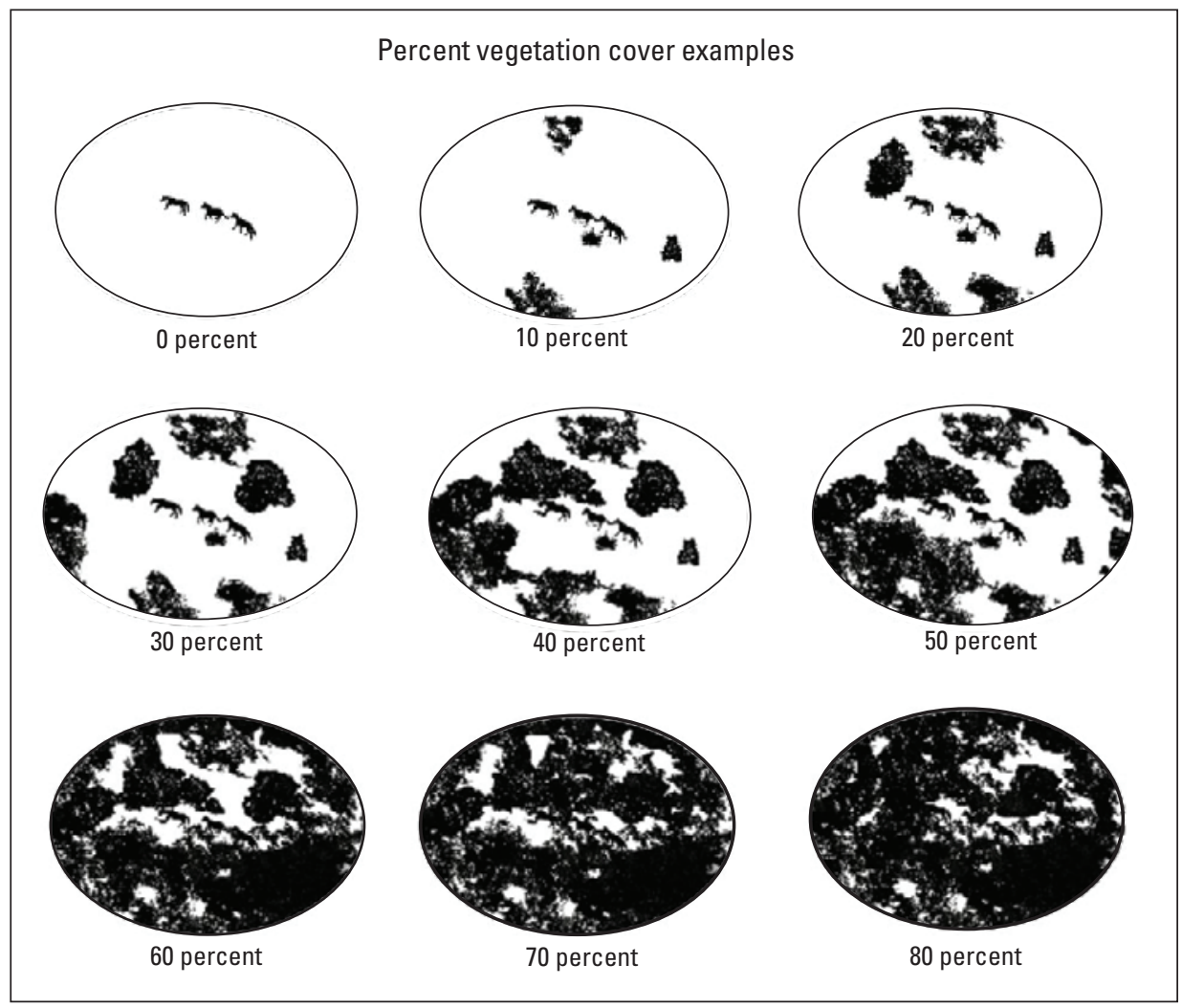

Figure 2.1. Diagram of examples of percent vegetation cover, from zero to 80 percent, for wild horse and burro aerial surveys. (Courtesy of J. Ransom). 


\section{Appendix 3. Blank Data Forms}

On the pages that follow are the "Flight Information Form" for all wild horse and burro (WHB) aerial survey flights, "Survey Datasheets for horses," and "Survey Datasheets for burros." Each form has a one-page set of instructions that can be printed on the back side of the form and can be used as an in-flight reference. 


\section{Instructions for Flight Information Form}

1) Use a new Flight Information Form at least once per day. You may need to use more than one if you include more than 1 substitute observer.

2) Record Date. Record State. If this flight takes place in more than one state, record the state where there was the majority of the area surveyed.

3) Record Areas surveyed Today: which HMA(s), WHT(s) and HA(s) these particular survey flights took place over.

4) Record the Pilot's name, and Company name. Circle one choice for where the pilot is sitting: in the left front (LF) or right front (RF) seat. Circle one category of pilot experience conducting horse or burro aerial surveys. Record Pilot's email and Telephone \# if you know it. You only need to record the pilot's experience level, email and telephone \# once per set of surveys in an HMA or complex.

5) List each Observer's name and Agency. Circle one experience level per observer (circle one: less than or equal to 5 days $(\leq 5)$, or more than 5 days (5+) that the observer has done aerial surveys for horses, burros, or other ungulates). You only need to record Agency, Experience, and Email once for each observer over a set of surveys in one HMA or complex.

There is room for the name, agency, and experience of one substitute observer that may come in on the $2^{\text {nd }}, 3^{\text {rd }}$, or $4^{\text {th }}$ flight of the day. Record these for any substitute observers that rotate through on flights. Work to minimize the total number of observers in an entire set of surveys.

6) List the Helicopter manager (if helicopter flight), and the Billee code, if known.

7) Circle Aircraft Type (helicopter / fixed wing); record the Model, and Tail \#.

8) If you are in a helicopter, circle whether the Doors are On or Off for this set of surveys. Be consistent for all flights in a set of surveys that are planned together: don't change doors on / off between flights.

9) Record the Hobbs meter start before first flight, and Hobbs meter stop after last flight. Record approximate Planned airspeed, in knots.

\section{FOR EACH FLIGHT:}

10) Make a new Flight \# each time you take off. Keep increasing the flight numbers for the entire set of surveys for an HMA, or complex of HMAs.

11) Fill in the Seating Chart with initials of each person in the left front (LF), right front (RF), left back (LB) and right back (RB) seats. Switch the backseat observers from left to right between flights. Make clear who was where; use $2^{\text {nd }}, 3^{\text {rd }}$, letters of last name, if needed. Every flight should have a pilot plus 3 observers.

12) Record the Takeoff Time (24-hour time, hours and minutes). When you arrive at the area to be surveyed, Record the Survey Start Time.

13) Record the Weather conditions just before or at the time of takeoff; you can fill this out while ferrying to the area that will be surveyed.

- Cloud \% is the percentage of overhead clouds covering the sky, to the nearest $10 \%$.

- Mist \% is the percentage of fog covering the ground, to the nearest $10 \%$.

- $\quad$ Circle whether the Lighting in general was Flat (cloudy overhead; no shadows visible on the ground) or was High contrast (sun was out; shadows were visible).

- Record the Temperature, circle whether that is in ${ }^{\circ} \mathrm{C}$ or ${ }^{\circ} \mathrm{F}$, and what the elevation was (in feet) at which that temperature was measured.

- $\quad$ Circle one Windspeed.

- Circle one Precipitation description.

14) Prepare a Survey Datasheet before you arrive at the survey area. Record observation data on the Survey Datasheets.

15) Record the Survey Stop Time on the Flight Information Form when you finish survey on this flight and begin to ferry back to the landing zone.

16) While you ferry back to the landing zone, review your Survey Datasheets for completeness.

17) At the end of the flight, record the Landing Time on the Flight Information Form.

18) Record the Overall Count Conditions for the flight you just finished, based on your impressions and other crew impressions.

19) At the end of each flight, entire crew should review the Survey Datasheets for completeness (during refueling, or at least after last landing). 


\section{Flight Information Form(1 per day) for Horse and Burro Aerial Survey}

Date $(\mathrm{mm} / \mathrm{dd} / \mathrm{yyyy})$

State

Areas Surveyed Today (HMAs, HSTs, HAs):

Pilot: Name Company Seat (circle) LF RF

Pilot experience (days of aerial survey): 1-5 6-10 $\quad>10 \quad$ E-mail

Telephone

Observer 1: Name Agency Experience (circle): $\quad<5 \quad 5+$

Observer 2: Name Agency

Observer 3: Name Agency Experience (circle): $\quad<5 \quad 5+$

Substitute Observer (if any): Agency Experience (circle): $\quad<5 \quad 5+$

Helicopter Manager Account /Billee:

Aircraft: (circle one): helicopter / fixed wing Model Hobbs Start Hobbs Stop

Planned speed Experience (circle): $\quad<5 \quad 5+$ Doors: On / Off Give each takeoff a New Flight Number, and fill in data below

Flight \#

Seating Chart:

Fill in initials $\rightarrow$

\begin{tabular}{|l|l|}
\hline LF & RF \\
\hline LB & RB \\
\hline
\end{tabular}

Times: 1.Takeoff 2. Survey start

3. Survey Stop 4.Landing Overall Count Conditions Excellent | Very Good | Good | Fair | Poor

Weather: Cloud (\%) Mist (\%) Light Flat or HiContrast Temp (C or F) at elev (ft) Wind (circle one) calm, light, moderate, high, gusty

\begin{tabular}{c|l|l|}
\hline Flight \# & LF & RF \\
Seating Chart: & & LB \\
Fill in initials $\rightarrow$ & & RB \\
\cline { 3 - 4 } & &
\end{tabular}

Weather: Cloud (\%) Mist (\%) Precipitation (circle one) none, mist, light rain, heavy rain, snow

Wind (circle one) calm, light, moderate, high, gusty

\begin{tabular}{c|l|l|}
\cline { 3 - 3 } Flight \# & LF & RF \\
Seating Chart: & & \\
\cline { 3 - 3 } Fill in initials $\rightarrow$ & & LB \\
\cline { 2 - 3 } & &
\end{tabular}

Weather: Cloud (\%) Mist (\%) Wind (circle one) calm, light, moderate, high, gusty

\begin{tabular}{|c|c|c|}
\hline Flight \# & $\overline{\mathrm{LF}}$ & RF \\
\hline $\begin{array}{l}\text { Seating Chart: } \\
\qquad \text { Fill in initials } \rightarrow\end{array}$ & LB & $\mathrm{RB}$ \\
\hline
\end{tabular}

Weather: Cloud (\%) Mist (\%) Wind (circle one) calm, light, moderate, high, gusty
Times: 1.Takeoff

3. Survey Stop

Overall Count Conditions Excellent | Very Good | Good | Fair | Poor

Light Flat or HiContrast Temp (C or F) at elev (ft) Precipitation (circle one) none, mist, light rain, heavy rain, snow

\section{Comments:}

Times: 1.Takeoff

3. Survey Stop Overall Count Conditions Excellent | Very Good | Good | Fair | Poor
2. Survey start

4.Landing

(C or F) at elev (ft)

Precipitation (circle one) none, mist, light rain, heavy rain, snow

Times: 1.Takeoff

3. Survey Stop

Overall Count Conditions Excellent | Very Good | Good | Fair | Poor

2. Survey start 4.Landing
(C or F) at elev (ft)

Precipitation (circle one) none, mist, light rain, heavy rain, snow 
2. For each takeoff, write the initials of each observer in the box at upper right, showing their seat. Survey start \& end times are just for "on survey" part of flight.

3. All observers should search for horses \& burros (not one recording, while others search). It is OK to divide datasheet between observers. Record Page \# of \#.

4. Use GPS unit to mark each Detection group's waypoint. Call out the waypoint number; it is the reference number for the Detection group.

\begin{tabular}{|c|c|}
\hline Waypoint \# & $\begin{array}{l}\text { Waypoint number for the DETECTION GROUP, from GPS. Call it out to the others to coordinate datasheet recording. Be vocal about waypoint numbers. } \\
\text { If there is more than one DETECTION GROUP marked by a single waypoint, record those with a lower case letter a, b, c, etc. For example, 35a, 35b, 35c. } \\
\text { If the Detection Group has more than one BAND, if you choose you can also record each numbered Band starting with "B" as B1, B2, B3, each with its own row, and } \\
\text { each with the same waypoint. You can record adults \& foals for each band, but one waypoint, and one set of these covariates applies to the entire detection group. }\end{array}$ \\
\hline Time & Record the 24-hour time, as hh:mm. \\
\hline Species & $\begin{array}{l}\text { Circle Horse or Burro. In an HMA with only one species, you note the 'default' species on the Flight Information Form comments. Recording Mules: Circle Burro, } \\
\text { but write "MULE" in comments. If there is a mixed group, record one data row for horses, one for burros, and one for mules, but use the same waypoint and a clear } \\
\text { comment that the animals were all together. For other species (i.e. elk, deer...), circle Other, and record count, and species in Comments (no covariates needed). }\end{array}$ \\
\hline Total Count & $\begin{array}{l}\text { Number of horses (or burros) in a DETECTION GROUP. Use the columns to signify adults and foals, i.e., } 6 \mid 1 \text { means } 6 \text { adults } 1 \text { foal (do not write } 7 \text { total). } \\
\text { If you are recording one row for each separate BAND within a Detection Group, just record the number of adults and foals for each Band. } \\
\text { If you record the adults and foals in each band, do not also write the total for all bands as a new line of data, or else those horses might be counted twice in data entry! } \\
\text { Take a Photo of any group bigger than } 20 \text { (preferably } 10 \text { or more), and update these numbers with reference to the photos, in the office. }\end{array}$ \\
\hline Detected by & $\begin{array}{l}\text { Circle each seat position to show who detected the Detection group on their own, without assistance from other observers. Circle all of the observer / observers } \\
\text { that detected the group independently. } \mathrm{LF}=\text { left front, } \mathrm{RF}=\text { right front, } \mathrm{LB}=\text { left back, } \mathrm{RB}=\text { right back. The key idea is that each observer should have independent } \\
\text { opportunities to detect or to miss each Detection group. It is normal for either observers to not detect some groups. So that all observers' sighting probabilities can be } \\
\text { estimated, back-seat observers must switch seats between flights. Front seat observer and pilot would preferably stay the same for the entire survey. }\end{array}$ \\
\hline Side of Ship & $\begin{array}{l}\text { Group location when first spotted. } \mathbf{L}=\text { Left of transect; } \mathbf{R}=\text { Right of transect; or } \mathbf{B}=\text { both (this can be for large groups that span the transect and could have been seen } \\
\text { from both rear seats (even if they were not actually seen), or a group that runs from one side to the other under aircraft and was available to be detected by both sides of } \\
\text { the aircraft at some point. Circle Center only in those rare occasions when the group was never visible to the rear seats because it was always right under aircraft. }\end{array}$ \\
\hline Activity & Move=moving; Still=Still when you first saw the horses or burros. \\
\hline Visual Field & $\begin{array}{l}\text { Circle one dominant type for the area of the horse detection group (all of the included bands) }+10 \mathrm{~m} \text { : Open= } 75 \% \text { of the ground is mainly grass, bare ground, veg } \\
\text { below hock height; Broken }=25 \% \text { or more of the ground is covered by vegetation from hocks to horse-head high); Tree }=25 \% \text { or more of the ground-covering } \\
\text { vegetation is taller than horse head. If shrubs and trees both cover } 25 \% \text { or more of the ground, choose Tree. }\end{array}$ \\
\hline $\begin{array}{l}\text { \% } \\
\text { Concealing } \\
\text { Vegetation }\end{array}$ & $\begin{array}{l}\text { Record the percentage of the horse-height space (focus on girth to ear height) obscured from your view by vegetation cover, in the area up to } 10 \mathrm{~m} \text { away from the } \\
\text { entire group (all of the included bands). Record to nearest } 10 \% \text {. "Concealing Vegetation" means plant material (stem, branch, foliage) of any combination of plant } \\
\text { species that obscure the horse-height space. If no value is filled in here, data will be entered as ZERO. }\end{array}$ \\
\hline Distance & Circle one approximate horizontal distance (in miles) from horse group to the nearest transect, even if it is a transect you haven't yet surveyed. \\
\hline Light Level & $\begin{array}{l}\text { Circle one light condition in the area of the horse group. Hi = High contrast, there was bright light and shadows at the horse group. Record horses in tree shade as } \\
\text { 'Hi.' Flat = Flat light, the entire area between aircraft and horse group was in shadow, with no distinct shadows; Shade= The horse group was in shade from a } \\
\text { particular cloud, cliff, or rock, but there were sunny places between the aircraft and the horses (so the horses were in the dark, but with light nearby) }\end{array}$ \\
\hline Topo Class & $\begin{array}{l}\text { Smooth }=\text { open terrain, little to no topography or rocks that could hide a horse. Rugged = rugged terrain with rocks or topography tall enough to hide a horse / burro. } \\
\text { Assess at the scale of an oval up to } 10 \mathrm{~m}(10 \text { yards }) \text { beyond the edge of the whole group. Smooth is the default choice that will be entered if you don't circle anything. }\end{array}$ \\
\hline $\begin{array}{l}\text { \% snow } \\
\text { covered }\end{array}$ & $\begin{array}{l}\% \text { of ground and shrubs within } 10 \mathrm{~m} \text { of the entire horse group (all of the included bands) that is covered in snow, to nearest } 10 \% \text {. } \\
\text { If no value is filled in here, zero is the default value, so data will be entered as ZERO. }\end{array}$ \\
\hline $\begin{array}{l}\text { Protocol } \\
\text { OK? }\end{array}$ & $\begin{array}{l}\text { This should be OK if all observers had independent chances to see the group; this means that no one spoke up or indicated about the group before the group was in line } \\
\text { with the rear seat crew's shoulders. OK is in bold font here because OK is the default choice... you only need to circle 'no' here if there was a violation. }\end{array}$ \\
\hline Comments & olations, notes about photography, etc. \\
\hline
\end{tabular}

Comments Can include: area (i.e., outside HMA...), color markings, explain protocol violations, notes about photography, etc. 


\section{WHB Survey Datasheet}

Flight \#

Data recorder name:

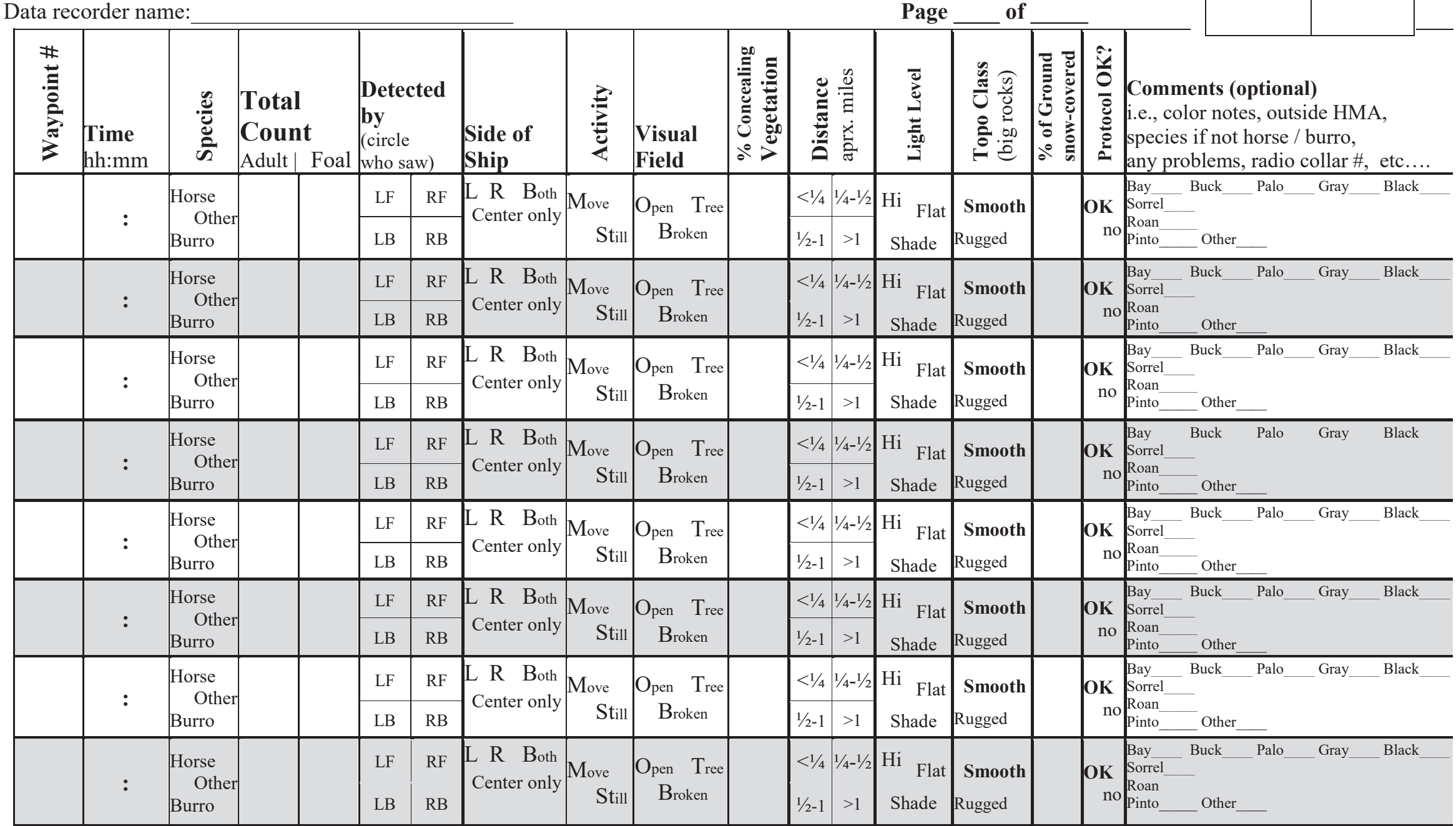

\section{(Start a new Flight \# and datasheet each time you take off)}

For this flight leg, write observers' positions in aircraft, use initials $\rightarrow$

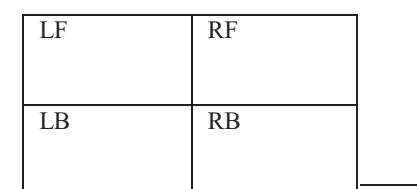



single flight. Keep increasing the flight numbers for the entire set of surveys for an HMA, or complex of HMAs.

2. For each takeoff, write the initials of each observer in the box at upper right, showing their seat. Survey start \& end times are just for "on survey" part of flight.

3. All observers should search for horses \& burros (not one recording, while others search). It is OK to divide datasheet between observers. Record Page \# of \#.

4. Use GPS unit to mark each Detection group's waypoint. Call out the waypoint number; it is the reference number for the Detection group.

\begin{tabular}{|c|c|}
\hline Waypoint \# & $\begin{array}{l}\text { Waypoint number for the DETECTION GROUP, from GPS. Call it out to the others to coordinate datasheet recording. Be vocal about waypoint numbers. } \\
\text { If there is more than one DETECTION GROUP marked by a single waypoint, record those with a lower case letter a, b, c, etc. For example, 35a, 35b, 35c. } \\
\text { If the Detection Group has more than one BAND, if you choose you can also record each numbered Band starting with "B" as B1, B2, B3, each with its own row, and } \\
\text { each with the same waypoint. You can record adults \& foals for each band, but one waypoint, and one set of these covariates applies to the entire detection group. }\end{array}$ \\
\hline Time & Record the 24-hour time, as hh:mm. \\
\hline Species & $\begin{array}{l}\text { Circle Horse or Burro. In an HMA with only one species, you note the 'default' species on the Flight Information Form comments. Recording Mules: Circle Burro, } \\
\text { but write "MULE" in comments. If there is a mixed group, record one data row for horses, one for burros, and one for mules, but use the same waypoint and a clear } \\
\text { comment that the animals were all together. For other species (i.e. elk, deer...), circle Other, and record count, and species in Comments (no covariates needed). }\end{array}$ \\
\hline Total Count & $\begin{array}{l}\text { Number of burros (or horses) in a DETECTION GROUP. Use the columns to signify adults and foals, i.e., } 6 \mid 1 \text { means } 6 \text { adults } 1 \text { foal (do not write } 7 \text { total). } \\
\text { If you are recording one row for each separate BAND within a Detection Group, just record the number of adults and foals for each Band. } \\
\text { If you record the adults and foals in each band, do not also write the total for all bands as a new line of data, or else those burros might be counted twice in data entry! } \\
\text { Take a Photo of any group bigger than } 20 \text { (preferably } 10 \text { or more), and update these numbers with reference to the photos, in the office. }\end{array}$ \\
\hline Detected by & $\begin{array}{l}\text { Circle each seat position to show who detected the Detection group on their own, without assistance from other observers. Circle all of the observer / observers } \\
\text { that detected the group independently. } \mathrm{LF}=\text { left front, } \mathrm{RF}=\text { right front, } \mathrm{LB}=\text { left back, } \mathrm{RB}=\text { right back. The key idea is that each observer should have independent } \\
\text { opportunities to detect or to miss each Detection group. It is normal for either observers to not detect some groups. So that all observers' sighting probabilities can be } \\
\text { estimated, back-seat observers must switch seats between flights. Front seat observer and pilot would preferably stay the same for the entire survey. }\end{array}$ \\
\hline Side of Ship & $\begin{array}{l}\text { Group location when first spotted. } \mathbf{L}=\text { Left of transect; } \mathbf{R}=\text { Right of transect; or } \mathbf{B}=\text { both (this can be for large groups that span the transect and could have been seen } \\
\text { from both rear seats (even if they were not actually seen), or a group that runs from one side to the other under aircraft and was available to be detected by both sides of } \\
\text { the aircraft at some point. Circle Center only in those rare occasions when the group was never visible to the rear seats because it was always right under aircraft. }\end{array}$ \\
\hline Activity & Move=moving; Still=Still when you first saw the horses or burros. \\
\hline Visual Field & $\begin{array}{l}\text { Circle one dominant type for the area of the burro detection group (all of the included bands) }+10 \mathrm{~m} \text { : Open }=75 \% \text { of the ground is mainly grass, bare ground, veg } \\
\text { below hock height; Broken }=25 \% \text { or more of the ground is covered by vegetation from hocks to burro-head high); Tree }=25 \% \text { or more of the ground-covering } \\
\text { vegetation is taller than burro head. If shrubs and trees both cover } 25 \% \text { or more of the ground, choose Tree. }\end{array}$ \\
\hline $\begin{array}{l}\text { \% Concealing } \\
\text { Vegetation }\end{array}$ & $\begin{array}{l}\text { Record the percentage of the burro-height space (focus on girth to ear height) obscured from your view by vegetation cover, in the area up to } 10 \mathrm{~m} \text { away from the } \\
\text { entire group (all of the included bands). Record to nearest } 10 \% \text {. "Concealing Vegetation" means plant material (stem, branch, foliage) of any combination of plant } \\
\text { species that obscure the burro-height space. If no value is filled in here, data will be entered as ZERO. }\end{array}$ \\
\hline Distance & $\begin{array}{l}\text { Circle one approximate horizontal distance (in approximate meters or yards) from burro group to the nearest transect, even if it is a transect you haven't yet surveyed. } \\
\text { If the distance is more than } \mathbf{4 0 0} \mathbf{~ m , ~ n o t e ~ t h e ~ a p p r o x i m a t e ~ d i s t a n c e ~ i n ~ t h e ~ c o m m e n t s ~ b o x . ~}\end{array}$ \\
\hline Light Level & $\begin{array}{l}\text { Circle one light condition in the area of the burro group. Record burros in tree shade as 'Hi.' Hi = High contrast, there was bright light and shadows at the burro group. } \\
\text { Flat = Flat light, the entire area between aircraft and burro group was in shadow, with no distinct shadows; Shade= The burro group was in shade from a particular } \\
\text { cloud, cliff, or rock, but there were sunny places between the aircraft and the burros (so the burros were in the dark, but with light nearby) }\end{array}$ \\
\hline Topo Class & $\begin{array}{l}\text { Smooth }=\text { open terrain, little to no topography or rocks that could hide a burro. Rugged = rugged terrain with rocks or topography tall enough to hide a horse / burro. } \\
\text { Assess at the scale of an oval up to } 10 \mathrm{~m}(10 \text { yards }) \text { beyond the edge of the whole group. Smooth is the default choice that will be entered if you don't circle anything. }\end{array}$ \\
\hline $\begin{array}{l}\text { \% snow } \\
\text { covered }\end{array}$ & $\begin{array}{l}\% \text { of ground within } 10 \mathrm{~m} \text { of the entire burro group (all of the included bands) that is covered in snow, to nearest } 10 \% \text {. Do not count snow on vegetation. } \\
\text { If no value is filled in here, zero is the default value, so data will be entered as ZERO. }\end{array}$ \\
\hline $\begin{array}{l}\text { Protocol } \\
\text { OK? }\end{array}$ & $\begin{array}{l}\text { This should be } \mathbf{O K} \text { if all observers had independent chances to see the group; this means that no one spoke up or indicated about the group before the group was in line } \\
\text { with the rear seat crew's shoulders. OK is in bold font here because } O K \text { is the default choice... you only need to circle 'no' here if there was a violation. }\end{array}$ \\
\hline Comments & Can include: area (i.e., outside HMA...), color markings, explain protocol violations, notes about photography, etc. \\
\hline
\end{tabular}


WHB Survey Datasheet For Burro Surveys (Start a new Flight \# and datasheet each time you take off)

Date: Flight \#

For this flight leg, write observers' positions in aircraft, use initials $\rightarrow$

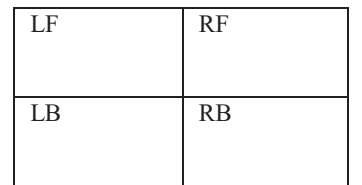

Data recorder name: Page ___ of

\begin{tabular}{|c|c|c|c|c|c|c|c|c|c|c|c|c|c|c|c|c|c|}
\hline \multirow[t]{3}{*}{ 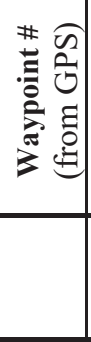 } & $\begin{array}{c}\text { Time } \\
\text { hh:mm }\end{array}$ & $\frac{\mathscr{c}}{\mathscr{E}}$ & \multicolumn{2}{|c|}{$\begin{array}{c}\text { Total } \\
\text { Count }\end{array}$} & \multicolumn{2}{|c|}{$\begin{array}{c}\text { Detected } \\
\text { by } \\
\text { (circle } \\
\text { who saw) } \\
\end{array}$} & $\begin{array}{l}\text { Side of } \\
\text { Ship }\end{array}$ & $\sum_{\substack{e\\
}}^{2}$ & \begin{tabular}{|c|} 
Visual \\
Field \\
\end{tabular} & 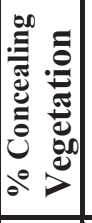 & \multicolumn{2}{|c|}{ 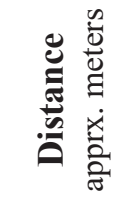 } & \multirow{3}{*}{ 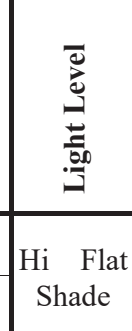 } & \multirow{3}{*}{ 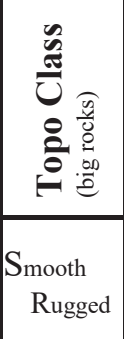 } & \multirow{3}{*}{ 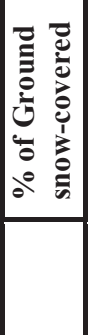 } & \multirow{3}{*}{ 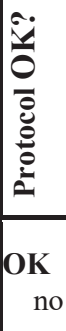 } & $\begin{array}{c}\text { Comments } \\
\text {...distance if }>400 \mathrm{~m}, \\
\text { outside HMA, color, problems, } \\
\text { radio } \#, \text { etc.... }\end{array}$ \\
\hline & \multirow[b]{2}{*}{ : } & \multirow{2}{*}{\begin{tabular}{|l} 
Horse \\
Other \\
Burro
\end{tabular}} & & & LF & $\mathrm{RF}$ & \multirow{2}{*}{$\begin{array}{l}\mathrm{L} \text { R Both } \\
\text { Center only }\end{array}$} & \multirow{2}{*}{$\begin{array}{l}\text { Move } \\
\quad \text { Still }\end{array}$} & \multirow{2}{*}{$\mid$\begin{tabular}{cc}
\multicolumn{2}{c}{ Broken } \\
Open & $T_{\text {ree }}$
\end{tabular}} & & $<100$ & $\begin{array}{l}100- \\
200\end{array}$ & & & & & Grey_Black__Pinto__ Other__ \\
\hline & & & & & LB & $\mathrm{RB}$ & & & & & $\begin{array}{l}200- \\
300\end{array}$ & $\begin{array}{c}300- \\
400\end{array}$ & & & & & \\
\hline & \multirow{2}{*}{ : } & \multirow{2}{*}{\begin{tabular}{|c|}
$\begin{array}{c}\text { Horse } \\
\text { Other } \\
\text { Burro }\end{array}$ \\
\end{tabular}} & & & LF & $\mathrm{RF}$ & \multirow{2}{*}{$\left|\begin{array}{ccc}L & R & \text { Both } \\
\text { Center only }\end{array}\right|$} & Move & \multirow{2}{*}{$\mid \begin{array}{c}\text { Broken } \\
\text { Open }\end{array}$} & & $<100$ & $\begin{array}{l}100- \\
200\end{array}$ & \multirow{2}{*}{$\begin{array}{l}\text { Hi Flat } \\
\text { Shade }\end{array}$} & \multirow{2}{*}{$\begin{array}{c}\text { Smooth } \\
\text { Rugged }\end{array}$} & \multirow{2}{*}{\multicolumn{2}{|c|}{\begin{tabular}{r|} 
OK \\
no
\end{tabular}}} & Grey_Black__Pinto__Other_ \\
\hline & & & & & LB & $\mathrm{RB}$ & & Still & & & $\begin{array}{l}200- \\
300\end{array}$ & $\begin{array}{l}300- \\
400\end{array}$ & & & & & \\
\hline & & & & & LF & $\mathrm{RF}$ & $\mathrm{L} \quad \mathrm{R}$ Both & Move & Broken & & $<100$ & $\begin{array}{l}100- \\
200\end{array}$ & Hi Flat & Smooth & & OK & Grey_B Black__Pinto__Other_ \\
\hline & : & Burro & & & LB & $\mathrm{RB}$ & & Still & Open $\quad$ Tree & & $\begin{array}{l}200- \\
300\end{array}$ & $\begin{array}{c}300- \\
400\end{array}$ & Shade & Rugged & & no & \\
\hline & & Horse & & & LF & RF & $\mathrm{L} \quad \mathrm{R}$ Both & Move & Broken & & $<100$ & $\begin{array}{l}100- \\
200\end{array}$ & Hi Flat & Smooth & & OK & Grey__Black__Pinto__ Other_ \\
\hline & • & & & & LB & $\mathrm{RB}$ & & Still & Open Tree & & $\begin{array}{l}200- \\
300\end{array}$ & $\begin{array}{l}300- \\
400\end{array}$ & & Rugged & & no & \\
\hline & & & & & LF & $\mathrm{RF}$ & L $\quad \mathrm{R}$ Both & Move & Broken & & $<100$ & $\begin{array}{l}100- \\
200\end{array}$ & Hi Flat & Smooth & & OK & Grey__Black__Pinto__ Other_ \\
\hline & $:$ & Burro & & & LB & $\mathrm{RB}$ & & Still & Open $\quad$ Tree & & $200-$ & $300-$ & Shade & Rugged & & no & \\
\hline & & Horse & & & LF & $\mathrm{RF}$ & L $\quad \mathrm{R}$ Both & Move & Broken & & $<100$ & $\begin{array}{l}100- \\
200\end{array}$ & Hi Flat & Smooth & & OK & Grey__Black__Pinto__Other_ \\
\hline & • & Burro & & & LB & $\mathrm{RB}$ & & Still & Open Tree & & $\begin{array}{l}200- \\
300\end{array}$ & $\begin{array}{ll}300- \\
400\end{array}$ & & Rugged & & no & \\
\hline & & Horse & & & LF & $\mathrm{RF}$ & $\mathrm{L} \quad \mathrm{R}$ Both & Move & Broken & & $<100$ & $\begin{array}{l}100- \\
200\end{array}$ & Hi Flat & $S_{\text {month }}$ & & OK & Grey_Black__ Pinto__ Other_ \\
\hline & : & Burro & & & LB & $\mathrm{RB}$ & & Still & Open $\quad T_{\text {ree }}$ & & $\begin{array}{l}200- \\
300\end{array}$ & $\begin{array}{c}300- \\
400\end{array}$ & Shade & Rugged & & no & \\
\hline & & Horse & & & LF & $\mathrm{RF}$ & L $\quad \mathrm{R}$ Both & Move & Broken & & $<100$ & $\begin{array}{l}100- \\
200\end{array}$ & Hi Flat & Smooth & & OK & Grey_Black__Pinto__Other_ \\
\hline & • & Burro & & & LB & $\mathrm{RB}$ & & Still & Open Tree & & $\begin{array}{l}200- \\
300\end{array}$ & $\begin{array}{l}300- \\
400\end{array}$ & & Rugged & & no & \\
\hline & & Horse & & & LF & $\mathrm{RF}$ & $\mathrm{L} \quad \mathrm{R} \quad \mathrm{B}_{\text {oth }}$ & Move & Broken & & $<100$ & $\begin{array}{l}100- \\
200\end{array}$ & Hi Flat & $S_{\text {month }}$ & & OK & Grey_Black__Pinto__ Other_ \\
\hline & • & Burro & & & LB & $\mathrm{RB}$ & er only & Still & Open $\quad \mathrm{T}_{\text {ree }}$ & & $\begin{array}{l}200- \\
300\end{array}$ & $\begin{array}{ll}300- \\
400\end{array}$ & Shade & Rugged & & no & \\
\hline & & Horse & & & LF & $\mathrm{RF}$ & L $\quad \mathrm{R}$ Both & Move & Broken & & $<100$ & $\begin{array}{l}100- \\
200\end{array}$ & Hi Flat & Smooth & & OK & Grey_Black__Pinto__ Other_ \\
\hline & • & Burro & & & LB & $\mathrm{RB}$ & & Still & Open Tree & & $\begin{array}{l}200- \\
300\end{array}$ & $\begin{array}{l}300- \\
400\end{array}$ & & Rugged & & no & \\
\hline
\end{tabular}




\section{Appendix 4. Pre-Survey Checklists}

\section{Action Checklist for Pre-Survey Consultation}

BLM WHB Program has provided guidelines that aerial inventories should use the most appropriate methods (BLM Instructional Memorandum 2010-057). As part of survey planning, district specialists (with guidance from state leads) should consult with the USGS aerial survey specialist or the BLM WHB research coordinator to determine those methods and plan flight paths. This checklist is meant to help you prepare for that consultation.

We recommend beginning the consultation process at least 2 months before survey dates, to meet survey planning needs (aviation safety, aircraft and crew scheduling, other compliance, etc.). However, if that is not possible, still seek consultation with USGS or the BLM research coordinator.

$\square$ 1. Be ready to discuss what survey method is appropriate. As of 2018, the two methods that have been tested on horses and account for undetected animals are "simultaneous double-observer" and "photo mark-resight" (Lubow and Ransom 2009; Lubow and Ransom 2016). Photo mark-resight is only appropriate in fairly small areas with a high degree of individual coloration in horses.

$\square$ 2. The timing of surveys should be consistent relative to past surveys. When should aerial surveys happen in this HMA / complex on a recurring basis?

$\square$ 3. Prepare GIS / Spatial information. Preferably these would be as shapefiles or a geodatabase, but .pdfs or .jpgs of historic survey maps can also be useful for planning.

- Polygon(s) of the total area that you plan to survey: this includes the entire HMA(s) / HA(s) / WHT(s) of interest, and also areas outside those boundaries where horses in that population might be, at the time of the survey.

- Lines showing barriers to horse movement: fences, major roads, ridges, major rivers, etc.

- Historic flight lines from past surveys that you think covered the area well

- Waypoints where horses have been seen during flights, or from ground observations

- Consider your preferred survey order across the landscape: How do you want to fly it?

- Potential refueling locations, if outside of an airport.

- Prepare a layer of polygons identifying areas where you expect to find no horses at all.

$\square$ 4. Contact USGS aerial survey biologist or BLM WHB research coordinator for consultation. In your initial email, please include:

- HMA / HA/ WHT names

- Approximate dates you expect to survey

- Background GIS / Spatial information files that will help with flight planning 


\section{Action Checklist for Horse Surveys, page 1 of 2}

\section{Survey Planning (times listed suggest how early to complete item, before surveys begin)}

$\square$ Consult with State lead, WHB specialists, and USFS biologists about where and when to survey.

$\square(2+$ mos.) Consult with USGS aerial survey specialist, or BLM WHB research coordinator about methods, flight planning, and data entry. Data collection must use a technique that provides correction for incomplete detection probability (currently: simultaneous double-observer or photo mark-resight). Plan on flying all HMAs in a complex together. Fly at the same time of year as the previous survey whenever possible.

$\square(\sim 1-2$ months) Schedule crew and aviation manager. Ensure / plan that crew is trained in all required aviation safety and survey methods.

$\square$ (-1 month) With USGS, lay out flight lines in GIS to ensure complete coverage of the area and to use barriers that minimize chances of horses moving between surveyed and unsurveyed areas.

$\square(1+$ month) Prepare Project Aviation Safety Plan (PASP) in consultation with aviation manager(s).

$\square$ (preferably 2 weeks, or longer) Project Aviation Safety Plan approved \& signed.

$\square(\sim 2$ weeks) Get the latest version of files for data entry + data management from USGS.

\section{Before Flights}

$\square$ (1 week) Give copies of PASP and maps to dispatch (days in advance), crew, and helicopter or fixed wing flight manager.

$\square$ (The day before pilot leaves the company/s base) Double check equipment requests with aircraft company (any antennas, cables, auxiliary power sources, etc.).

$\square$ (1 week \& daily prior to flight) Review which areas you are going to survey and in which order. Double-check your plan and confirm with dispatch whether or not there is going to be other known aircraft in the area at the same time (fixed wing, helicopter, military, unmanned aircraft systems). Communicate with any other known aircraft.

$\square$ ( $\leq 3$ days) Upload survey area boundaries file into pilot/s GPS unit.

$\square$ ( $\leq 3$ days) Review flight objectives, crew responsibilities.

$\square$ ( $\leq 3$ days) Get list of radio-collared horse frequencies and last known locations, if applicable.

$\square(\leq 3$ days) Review the data sheets (Flight Information Form, Survey Datasheet) and directions for Survey Datasheet sheet with all flight crew.

$\square$ ( $\leq 3$ days) Synchronize watches and cameras with GPS unit. Check / install fresh batteries.

\section{Each Dav of Flight}

$\square$ Prepare 2 navigation tablet or GPS to show and record the flight track. Always use a backup unit.

$\square$ Review fuel load to meet flight objectives. Confirm that all gauges are functional.

$\square$ Make sure pilot/aircraft has proper radio frequencies

$\square$ Test radio reception with dispatch / flight follower before survey flight

- Aircraft Manager: Do not survey if the aircraft radio cannot talk with dispatch / flight follower / other aircraft involved with the survey.

$\square$ Wash the windows to improve visibility (use high-quality spray and towels).

$\square$ Review all observers/ duties and data collection methods. Distribute forms, pencils, clipboards.

$\square$ Review covariate measures with crew, especially \% concealing vegetation.

$\square$ Review in-flight data collection methods.

$\square$ Test intercom system (ICS) in aircraft; replace malfunctioning helmets/headsets if needed.

$\square$ Test radio-telemetry receiver and antennas, if applicable.

$\square$ Ensure the track $\log$ can be downloaded after the flight.

$\square$ Pilot will conduct an aviation pre-flight safety briefing (include visual flight rule (VFR) cruising altitudes; known aerial hazards) and mission briefing.

$\square$ Speak up if you feel uncomfortable about anything during pre-flight checks or in-flight, and don/t hesitate to ground the flight. 


\section{Action Checklist for Horse Surveys, page 2 of 2}

\section{Flight Guidelines}

$\square$ Survey the HMA (or complex) completely, following flight transect lines.

$\square$ Speeds: $\sim 50-60 \mathrm{kt}$ (burro), 55-70 kt (horse), 95-130 kt in fixed wing. Actual speed will vary.

$\square$ Call dispatch at take-off \& landing; confirm that AFF is operational, flight follow if needed.

$\square$ Communicate with any other aircraft in the area about locations.

口 Data recorder: Complete a new Flight Information Form at each take-off (new flight \#) and start a new set of Survey Datasheets at each takeoff, using the new flight number.

$\square$ If there is a digital audio recorder, state the exact time early in the flight for synchronization.

$\square$ Call out hazards to the pilot during flight: towers, powerlines, aircraft, terrain, birds, etc.

\section{In-Flight Data Collection Methods (review these as a group during pre-flight briefing)}

$\square$ Stay quiet about any horse group you see until it is perpendicular to the ship/s path.

$\square$ Wait until the group is just past perpendicular, then speak up! For every group observed, tell the data recorder whether you saw the group before it was called out (that/s the definition of detecting it /independently/).

$\square$ If someone spoke up before the group was past the back observers, tell the recorder there wasn/t an independent chance to detect the group ("Protocol OK?" = no).

$\square$ Work together to count and determine the number of adults and foals. Tell the pilot to not split up the group, if possible. Take photo to help count \& classify, if needed.

$\square$ If needed, ask pilot to encourage the group away from unsurveyed areas while maintaining a safe above ground level (AGL) flight altitude.

$\square$ Help the data recorder by calling out the data: Waypoint \#, Species, Count, Who saw it independently, Side of Ship (Left / Right / Both / center only), Activity (Moving / Still), Topo Class (Smooth / Rugged), Visual Field Type (Open / Broken / Tree), \% Concealing Veg $(0-100 \%), \%$ snow cover (0-100\%), Light (Flat / High contrast / Cloud), and Distance $(<1 / 4 \mathrm{mi}, 1 / 4-1 / 2 \mathrm{mi}, 1 / 2-1 \mathrm{mi},>1 \mathrm{mi}$ for horses, $<100 \mathrm{~m}, 100-200 \mathrm{~m}, 200-300 \mathrm{~m}, 300-400 \mathrm{~m},>400 \mathrm{~m}$ for burros).

$\square$ Always take photos for groups larger than 20 horses; preferably for groups more than 10. Hint: take a photo of your shoe between groups to separate horse groups.

$\square$ Be sure that all data are complete for each horse group detected.

$\square$ Maintain high cruising altitude during ferry flight to and from survey area. Pilot calls dispatch when entering or leaving survey area.

\section{At end of Flight / during refueling}

$\square$ Data recorder fills in Weather Conditions at Landing.

$\square$ Everyone: check over flight data forms in detail, for completeness.

$\square$ Recharge / change camera, laptop, and GPS unit batteries, if needed.

$\square$ Post-flight briefing: confer and note what worked and what didn/t on this survey.

$\square$ Download digital files: flight path, waypoints, photos.

\section{In Office as Soon as Possible after Flight. Deadline to send data to USGS is 6 weeks}

$\square$ Review and rename photos. update numbers of adults and foals in each group on data sheets.

$\square$ Enter all data into most current excel format (or, eventually, database)

$\square$ Verify that all data are entered completely and correctly (at other time after data entry)

$\square$ Send copies of all digital files to USGS aerial survey biologist or BLM WHB research coordinator, and to your district GIS specialist.

- Completed data entry file

- All flight lines and waypoint files, using the standardized file naming

- scanned (.pdf) copies of data forms

- Data photo files, with standard file naming

After Population Estimates Draft is sent to Districts, but Before Report is Public

$\square$ Discuss population estimates and interpretation with USGS and BLM research coordinator. 


\section{Checklist for Survey Equipment, page 1 of 2}

\section{For Pilot/s GPS unit}

$\square$ Planned flight lines and navigation waypoints for transfer to pilot/s GPS.

$\square$ Laptop or other computer that can transfer background map data files to pilot/s GPS unit (DNRGarmin, ExpertGPS, or other software loaded and ready).

$\square$ Data transfer cable that fits pilot/s GPS device (most often USB to mini-USB).

\section{Each Crew Member}

$\square$ Personal Protective Equipment (PPE). For helicopters: Nomex flight suit, Nomex gloves, leather boots, helmet, earplugs. Reference Aviation Life Support Equipment (ALSE) April 2018 Chpt 2 Personal Protective Equipment. None required for fixed wing flights above 500/ AGL.

$\square 10$ Essentials plus: food \& water, knife, matches, fire starter, map \& compass, extra warm clothes, raingear, space blanket, first aid kit, flashlight...plus sealable air sickness bags.

$\square$ Personal locator beacon (optional).

$\square$ Lightweight clipboard \& field forms, pencils, watch or clock synchronized to GPS.

\section{In the Aircraft}

Safety reminder for doors-off flights, everything shall be secured (attached to an observer), so that nothing can fall out the door. Reference ALSE, 2.4 Aircrew Member Secondary Restraint System.

$\square$ List of agency radio frequencies and map of repeaters

$\square$ HMA / WHT maps (one for front seat, one for back seat)

$\square$ Flight plan map approximate times to fly each surveyed area

$\square$ Field forms

- Pencils - for each observer

- Lightweight clipboards -1 per observer.

- Large rubber bands to hold down papers on clipboard, if desired

- Flight Information Forms (1 per day of flight)

- Survey Datasheets for each day of survey. 10 groups per page.

$\square$ GPS unit or GPS-enabled laptop / tablet computer

- Fully charged, with survey unit boundaries and planned flight paths visible

- External GPS unit or antenna, and connections, tested and operating (if needed)

- Device should display and record the flight path, while in flight

- Spare batteries, fully charged

- Cloth, to wipe screen

- AC power adapter for use in helicopter (optional)

- Memory key / thumb drive, for data backup or transfer after flight (optional)

- Carrying case (optional)

- GIS security key attached to laptop (if necessary)

$\square$ Second GPS unit, to record backup of the flight path

- External GPS antenna and cables, if needed

- Spare batteries, fully charged

- The aircraft/s GPS may be used for this, if it records tracks

$\square 3$ timing clocks or large-faced digital watches — one for each observer

- Use GPS unit to synchronize all clocks every day

$\square$ Digital camera with image-stabilized zoom, synchronized to the GPS unit, on sports mode setting

- Spare camera batteries

- Spare compact flash memory card

$\square$ Duct tape (and/ or zip ties and/ or velcro strips) to secure loose cables

$\square$ Copy of Project Aviation Safety Plan

$\square$ Printed copy of the Quick-Guide to Double-Observer Surveys

$\square$ Handheld radio (if applicable) 


\section{Checklist for Survey Equipment, page 2 of 2}

\section{In the Aircraft, continued:}

$\square$ Cell phone and / or satellite phone (Optional)

$\square$ Equipment bag (Optional)

$\square$ Scissors (Optional)

$\square$ Digital audio recorder (Optional), synchronized to GPS time

- Olympus DS-30 digital voice recorder and user/s guide

- CellSet 5000H adapter

- $1 / 8$ " male to $3 / 32$ " female stereo adapter

- 2 extra AAA batteries

$\square$ Telemetry gear, if needed

- Scanning VHF Receiver and antenna

- UHF Terminal and antenna, if needed

- Spare batteries, charged

- Ear pieces

- Adapters for aircraft avionics

- Coaxial cables (10 ft)

- Individual horse radio frequency list, collar colors and Horse IDs or other codes

\section{At the Landing Zone, Helicopter Flights}

$\square$ Helicopter manager (if this person is not an aircrew member/observer).

$\square$ Helispot safety items:

- Radio; fire extinguisher; wind indicator; crash kit; traffic control equipment, dust abatement, if needed

๑ Project Aviation Safety Plan, Departmental Manual, BLM Manual, IHOG, \& other policy guides

$\square$ Aviation Mishap Response Guide and Checklist

$\square$ Appropriate account numbers for OAS-23 forms

$\square$ Survey area maps

$\square$ Scale

$\square$ Load calculation book

$\square$ Passenger manifest pages

$\square$ Safety briefing cards

$\square$ Extra helmet and Nomex flight suit

\section{At dispatch}

$\square$ Confirm Automated Flight Following (AFF) capability

$\square$ Completed and signed Project Aviation Safety Plan

- Aviation Mishap Response Guide and Checklist

- Survey area maps, with schedule for which areas will be surveyed when

- Coordinate list (this can be coordinates for reference points within the survey area; to be used for quick location reference in the event of an emergency) 


\section{Appendix 5. Quick-Guide to Double-Observer Surveys}

This quick-guide serves a reference guide that should be consulted immediately before take-off with the pilot and all aircrew members/observers. It is designed to be printed and carried on board the aircraft during each survey. 


\section{Simultaneous double-observer wild horse \& burro aerial survey SOPs}

\section{Safety is your top priority!!!}

- Safety is your first, second, and third priorities; the survey is a distant fourth. Practice extreme safety.

- If you see, smell, hear, feel, or notice anything you don't like, ground the flight. Call out hazards in air.

- If you feel time- or other pressure to get the survey done, that should raise your hackles. Be extra safe.

- With doors off, you MUST tie down ALL equipment with velcro or string: waterbottle, GPS, clipboard.

\section{One-minute review of methods}

- Everyone searches independently. Only call out groups once they are past your 3 or 9 o'clock.

$\circ$ Everyone in the aircraft must have a fair chance of finding the group themselves.

- Don't get cues from other people (for example you see the front seat observer turning his/her head to look for a group). If you get cued for any reason, call the group "off-protocol."

- Be honest about groups you don't see.

O If you only saw the group after it was called out, you missed it.

- It's not a competition, and you will miss some obvious groups. That's ok.

- If you're not honest about misses, we will underestimate the horse/burro population.

- Discuss "\% concealing vegetation" and "Distance" early so everyone classifies them the same.

$\circ$ Do an eyeball calibration by asking pilot to drop a GPS waypoint at landing zone and hover $1 / 4,1 / 2$, and 1 mile away (or 100 meters, $200 \mathrm{~m}$, and $400 \mathrm{~m}$ away for a burro survey).

- Record every covariate for every group, even for groups that are "off-protocol."

- Rotate backseat observers (left-right) each fuel cycle.

$\circ$ Do not rotate observers between the front seat and backseat. Only have 1 person record data.

- Photograph all large groups. Video very large groups.

\section{Common mistakes}

1. Burros are recorded in segments of 100 meters $(=1$ football field $)$, not $1 / 4$ mile segments.

2. Broken $=\underline{\text { vegetation }}$ with tall shrubs or young trees that are above horse's hocks but not above head.

3. Rugged $=$ topography. Were any horses ever hidden by topography as you approached? If yes, it's rugged.

4. Distance is group's perpendicular distance from the transect line, not distance from the aircraft.

5. Shaded light is when groups are fully in shadow with bright light in between the aircraft and shade. In trees, only record if shade itself affects detection (e.g. burros hiding in shade of tree on a hot sunny day).

6. Side of ship: both is a group that's spread out so $\underline{b o t h}$ backseat observers can see it; center only is a group directly under the aircraft (between the skids of the helicopter) that neither backseat observer can see.

7. If a backseat observer sees a group on the wrong side of the ship: mark the group as "off-protocol" if that was the only person who saw the group.
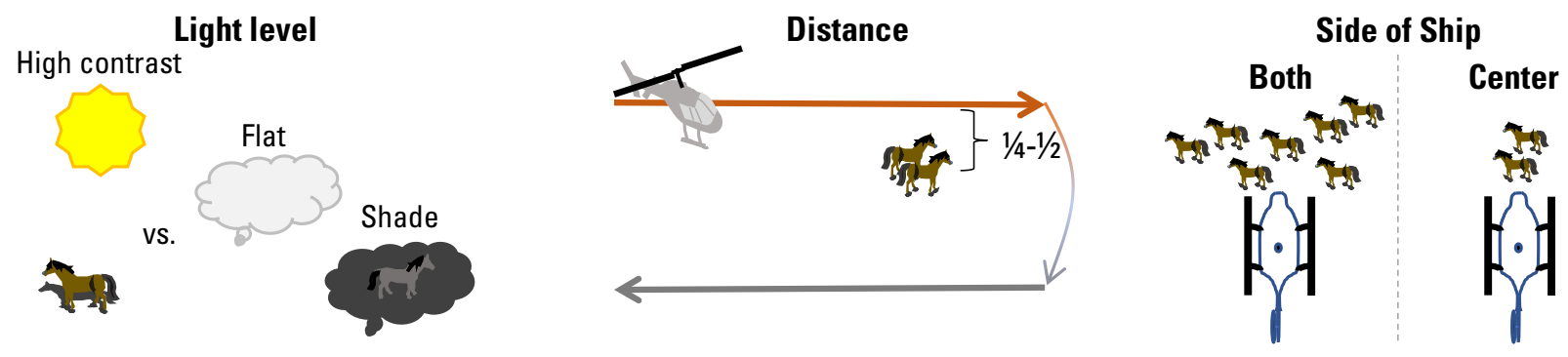


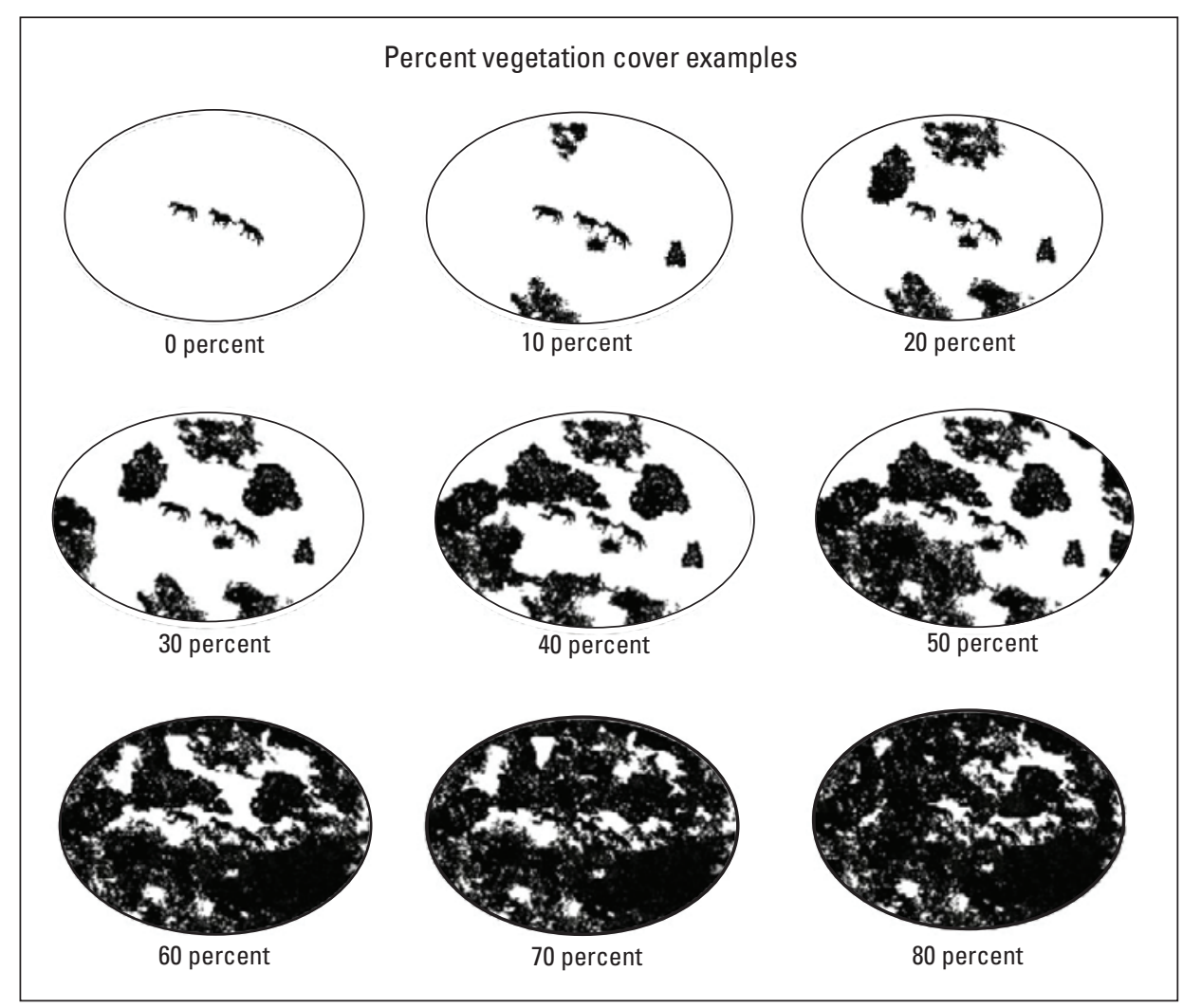

Wait until horses are past your 3 or 9 o/clock, and almost at your 4 or 8 o/clock, before calling out a group.

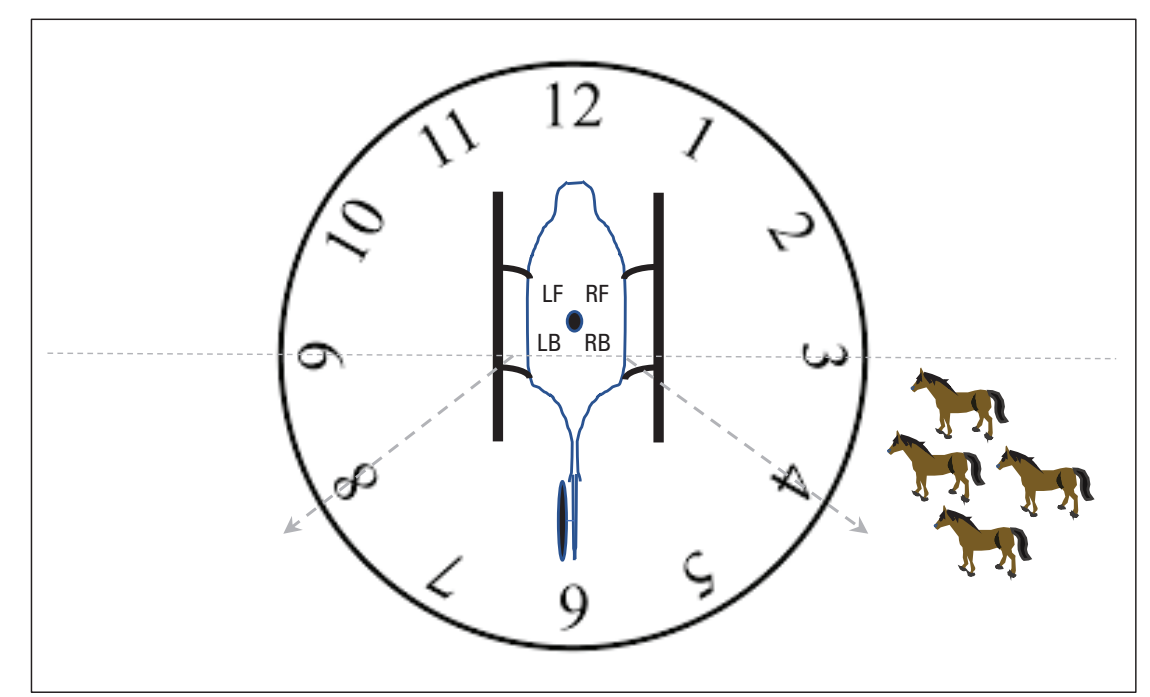

Tips

- If you/re flying doors off, get 2 clipboards and use large rubber bands to secure datasheets to front $\&$ back of each clipboard. That way you have 4 datasheets to fill in before needing to fumble with papers.

- Anti-nausea medicines: Zofran (prescription) is best. Meclizine/Bonine is less drowsy than Dramamine.

- Always have a ziplock bag ready in case you get airsick. Have snacks handy to fend off nausea.

- Sync camera and watches to GPS time. Matching up photos with wrong time stamp is a nightmare.

- Flying is exhausting. Make sure you get good sleep, and avoid long drives if you can. 
Publishing support provided by the Science Publishing Network, Denver Publishing Service Center

For more information concerning the research in this report, contact the Center Director, USGS Fort Collins Science Center

2150 Centre Ave., Bldg. C

Fort Collins, CO 80526-8118

(970) 226-9100

Or visit the Fort Collins Science Center web site at: https://www.usgs.gov/centers/fort 
